INTERNATIONAL MONETARY FUND

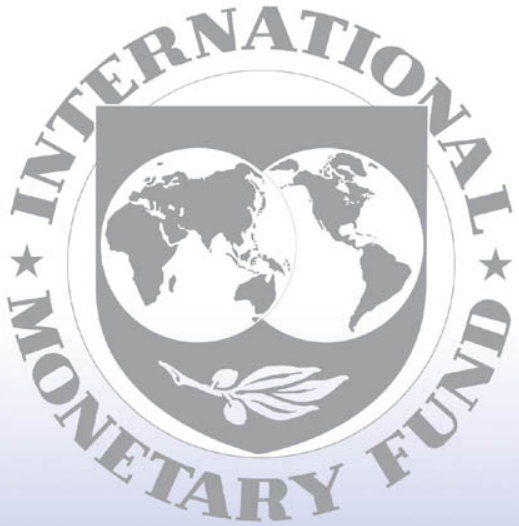

Staff

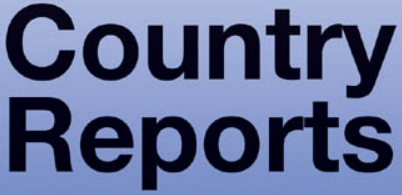




\section{Grenada: Third Review Under the Three-Year Arrangement Under the Poverty Reduction and Growth Facility, Requests for Modification of Quantitative Performance Criterion and Augmentation, and Financing Assurances Review-Staff Report; Staff Supplement; and Press Release on the Executive Board Discussion}

In the context of the third review under the Poverty Reduction and Growth Facility, requests for modification of quantitative performance criterion and augmentation, and the financing assurances review for Grenada, the following documents have been released and are included in this package:

- $\quad$ The staff report for the Third Review Under the Poverty Reduction and Growth Facility, Requests for Modification of Quantitative Performance Criterion and Augmentation, and Financing Assurances Review, prepared by a staff team of the IMF, following discussions that ended on March 25, 2009, with the officials of Grenada on economic developments and policies. Based on information available at the time of these discussions, the staff report was completed on May 20, 2009. The views expressed in the staff report are those of the staff team and do not necessarily reflect the views of the Executive Board of the IMF.

- $\quad$ A staff supplement on the Debt Sustainability Analysis.

- $\quad$ A Press Release summarizing the views of the Executive Board as expressed during its June 3, 2009, discussion of the staff report that completed the request.

The document listed below will be separately released.

Letter of Intent sent to the IMF by the authorities of Grenada.*

Supplementary Memorandum of Economic Policies by the authorities of Grenada*

Supplementary Technical Memorandum of Understanding*

*Also included in Staff Report

The policy of publication of staff reports and other documents allows for the deletion of market-sensitive information.

Copies of this report are available to the public from

$$
\begin{aligned}
& \text { International Monetary Fund } \bullet \text { Publication Services } \\
& 70019^{\text {th }} \text { Street, N.W. } \bullet \text { Washington, D.C. } 20431
\end{aligned}
$$

Telephone: (202) 623-7430 • Telefax: (202) 623-7201

E-mail: publications@imf.org •Internet: http://www.imf.org

\section{International Monetary Fund Washington, D.C.}


This page intentionally left blank

(CInternational Monetary Fund. Not for Redistribution 
INTERNATIONAL MONETARY FUND

GRENADA

\title{
Third Review Under the Three-Year Arrangement Under the Poverty Reduction and Growth Facility, Requests for Modification of Quantitative Performance Criterion and Augmentation, and Financing Assurances Review
}

\author{
Prepared by Western Hemisphere Department \\ (In consultation with other departments)
}

Approved by José Fajgenbaum (WHD) and Aasim Husain (SPR)

May 20, 2009

Context: The global slowdown and financial turmoil have negatively affected tourism receipts, FDI, and remittances. The banking system has remained resilient thus far, but the intervention of the Trinidad and Tobago-based CL Financial Group has increased financial sector uncertainty. Growth is projected to slow markedly in 2009, as almost all FDI-financed tourism projects are on hold.

Arrangement: On April 17, 2006, the Executive Board approved a PRGF Arrangement for SDR 10.53 million (90 percent of quota). In July 2008, the Board completed the first program review, augmented access to 102.5 percent of quota to help mitigate the impact of food and fuel price shocks, and extended the arrangement by one year to April 16, 2010. The second review was completed in December 2008.

Program performance: All end-2008 quantitative targets were met. Meeting structural benchmarks on submitting investment legislation and a new Excise Bill and on completing a Country Poverty Assessment has been delayed by the time required to consult stakeholders, to finalize the list of excisable goods, and to complete the technical work and drafting, respectively.

Review: In the attached Letter of Intent and Supplementary Memorandum of Economic Policies, the authorities elaborate on their policies for 2009 and propose quantitative performance criteria and structural benchmarks through 2009. They also request completion of the review, modification of the end-June 2009 performance criterion on the primary balance (by 1.4 percent of GDP), and augmentation of access to 140 percent of quota, with an additional SDR 4.39 million available upon completion of the third and fourth reviews.

Missions: A mission visited St. George's during March 16-25, 2009 and met with Prime Minister Thomas, Minister of Finance Burke, Permanent Secretary Antoine, other senior officials, and private sector representatives. The mission overlapped with a mission conducting the ECCU Common Policies discussions, and comprised Ms. Pattillo (Head), Messrs. Monroe, Kang (all WHD), and Das (SPR), with input from Ms. Kaltani (SPR). 
I. Recent Developments and Outlook

A. Recent Developments .............................................................................

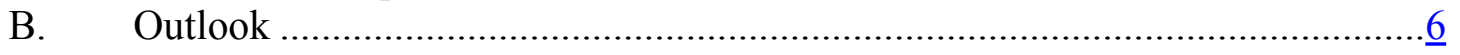

II. Performance Under the Program ……………....................................................

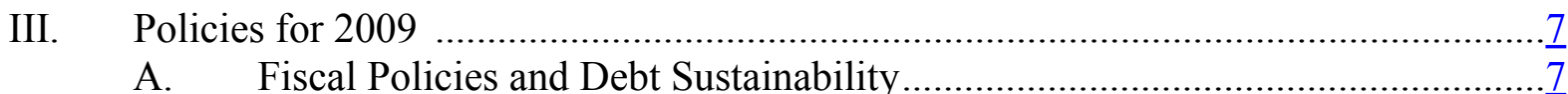

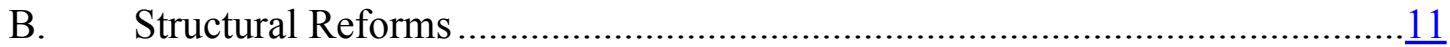

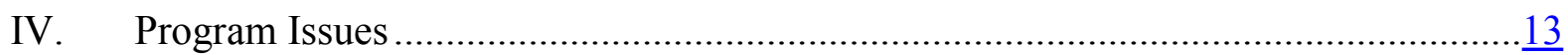

A. Program Design ....................................................................................13

B. Program Risks and Financing Assurances .......................................................14

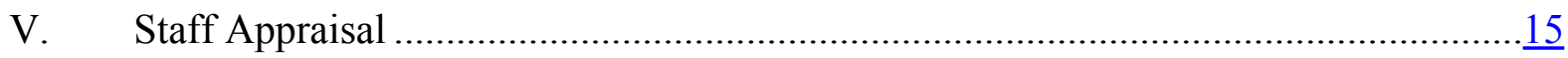

Figures

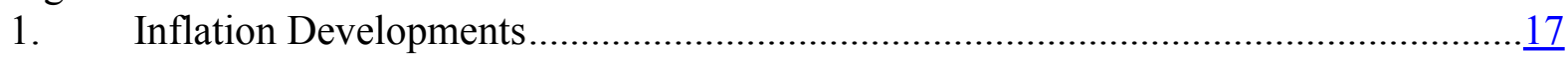

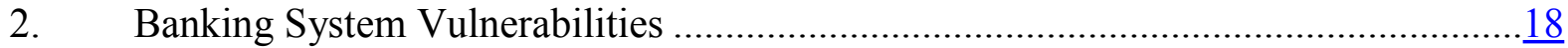

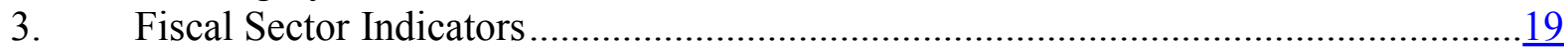

4. Competitiveness Indicators .................................................................................

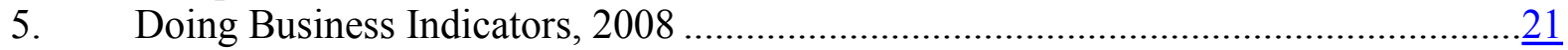

Tables

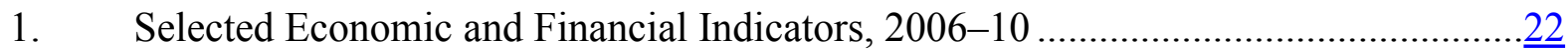

2. Central Government Finances, 2006-09 (in millions of EC dollars) ..........................23

2a. Central Government Finances, 2006-09 (in percent of GDP)...................................

3. Summary Accounts of the Banking System, 2006-10 ….........................................

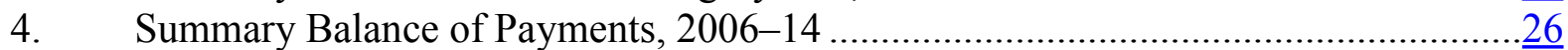

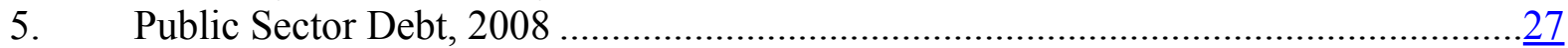

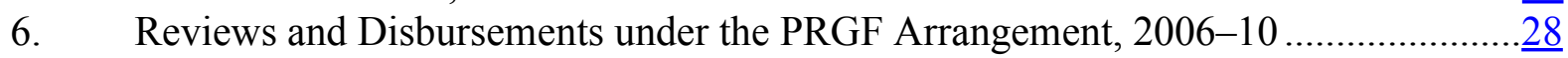

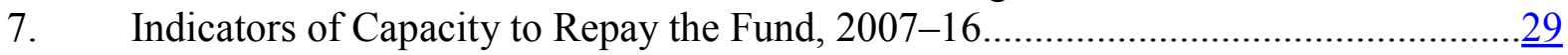

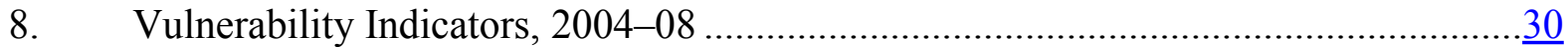

Annex

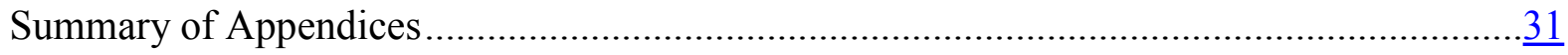

Attachments

I. Letter of Intent .........................................................................................

II. Supplementary Memorandum of Economic Policies …………………………….....

III. Supplementary Technical Memorandum of Understanding ………………….........44 


\section{EXECUTIVE SUMMARY}

The global economic slowdown and financial turmoil are markedly slowing economic activity, chiefly through a weakening of tourism receipts, FDI, and remittances. Almost all FDI-financed tourism projects are on hold. Real GDP is projected to decline by 0.7 percent in 2009 and to recover slightly in 2010. Inflation is projected to fall to 2.1 percent by the end of 2009 , as declines in world food and fuel prices feed through. Commercial banks have remained resilient, but the intervention of the Trinidad and Tobago-based CL Financial Group is increasing financial sector uncertainty.

All end-December 2008 quantitative targets were met, reflecting the authorities' strong remedial fiscal measures in the second half of 2008. In particular, the authorities met the target on the primary deficit excluding grants, which fell from 5.1 percent of GDP in the first half to 2.4 percent of GDP in the second half of the year. They reduced arrears older than 60 days to only EC\$8.4 million (0.5 percent of GDP), meeting this performance criterion by a significant margin. Capital expenditure of 12.5 percent of GDP in 2008 was higher than the programmed level of 10.7 percent of GDP, but did fall by 3 percentage points of GDP from the first to the second half of 2008.

Meeting some structural benchmarks is taking longer than envisaged. The performance criterion on initiating reorganization or liquidation of Capital Bank was met in November 2008. A Debt Management Unit was established in January 2009. However, benchmarks on submitting investment incentives legislation and completing the Country Poverty Assessment are not expected to be met until end-May and August 2009 respectively. The government has announced an introduction date for the VAT of February 1, 2010. Preparations are proceeding well, aided by CARTAC technical assistance; the authorities submitted the VAT Bill to Parliament in April on schedule, and plan to submit the Excise Bill in August 2009 rather than in April as previously envisaged.

The revised fiscal program envisages a primary deficit of 4.4 percent of GDP in 2009. Due to the global slowdown, shortfalls are expected in trade-related taxes, grants, and divestment proceeds, and the government has adjusted primarily by revising capital spending downward from 10 to 8.9 percent of GDP. To address the impact of the slowdown, capital projects that provide the greatest economic stimulus will be accelerated, while transfers to vulnerable groups will increase. Despite the spending cuts, the original end-June 2009 primary balance target is no longer attainable.

The government intends to continue and accelerate structural reforms, while sharpening their focus. The government will appoint a separate Registrar of Companies as a key step in their action plan to improve Doing Business Indicators. They are also pushing forward with steps to prepare for VAT introduction, to implement a customs Fraud Control Plan, and to improve the efficiency of public procurement. Completion of the Country Poverty Assessment is an essential input to preparing a full PRSP.

The government may seek a US\$50-80 million (7-12 percent of GDP) concessional loan from the Export-Import Bank of China to build a luxury hotel in a joint venture. With the already high debt level (108.6 percent of GDP at end-2008), the loan could jeopardize the key objective of reducing debt and is not accommodated within the program ceiling on bilateral concessional debt.

Program risks are significant. The impact of financial turmoil and the global economic slowdown on tourism, FDI, growth, revenue, and grants could be stronger than expected. In addition, the intervention of CL Financial Group could affect financial sector confidence, and the possible loan from China would undermine debt reduction objectives. 


\section{RECENT DEVELOPMENTS AND OUTLOOK}

1. Grenada has made good progress with its home-grown economic program, which was launched with the 2006 budget and is supported by the PRGF. However, the sharp slowdown in global growth and the continuing global financial crisis have had a large adverse impact on growth, the external position, and fiscal revenue. As a result, it has been necessary to revise the policy framework for 2009 to address these challenges, while maintaining the key objectives of reducing public debt, reinvigorating growth through structural reforms, reducing vulnerabilities, and furthering the nation's social development agenda.

\section{A. Recent Developments}

2. Economic growth has declined sharply reflecting the global slowdown. Real GDP growth slowed to 0.3 percent in 2008 , from 4.5 percent in 2007, as tourism receipts and FDI weakened. Tourist stayover arrivals declined by about 5 percent in 2008. Almost all FDI-financed tourism investment projects have been put on hold due to financing difficulties, and as a result, the construction sector, which accounted for about 10 percent of real GDP in 2007 , contracted by more than 14 percent in 2008. Some large employers and many hotels have laid off or are rotating staff. ${ }^{1}$

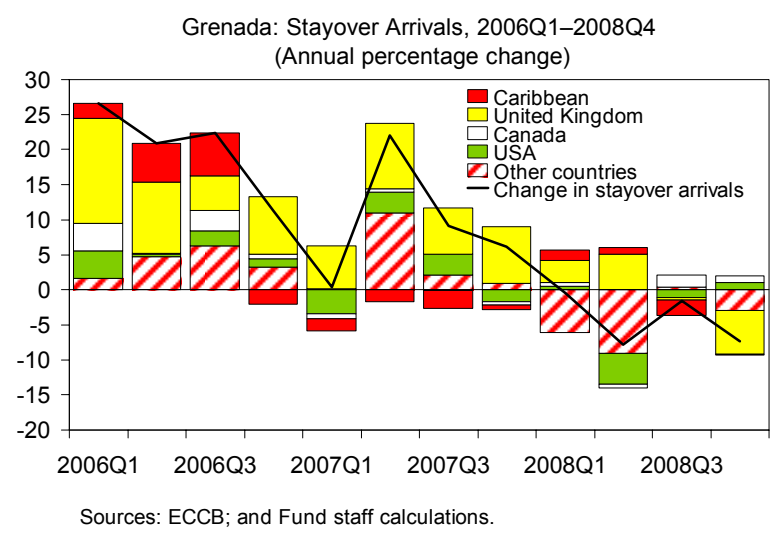

3. The external current account deficit increased slightly in $\mathbf{2 0 0 8}$ due to high world fuel and food prices and lower tourism receipts and exports, which were partially offset by increased official grants. The real effective exchange rate appreciated by $61 / 2$ percent through December 2008, reflecting the appreciation of the U.S. dollar, to which the Eastern Caribbean dollar is pegged, against other major currencies.

\footnotetext{
${ }^{1}$ The authorities estimate that the unemployment rate was around 25 percent in 2008 (based on preliminary data from the Country Poverty Assessment), and they project that it will rise significantly in 2009.
} 
4. Inflation declined to 2.8 percent by March 2009, after peaking at 9.3 percent in July 2008. The inflation path reflects the trajectory of world fuel and food prices and the dominant weight of these items in the CPI. Core inflation (excluding food and energy) was only 1.5 percent through March 2009 (Figure 1).

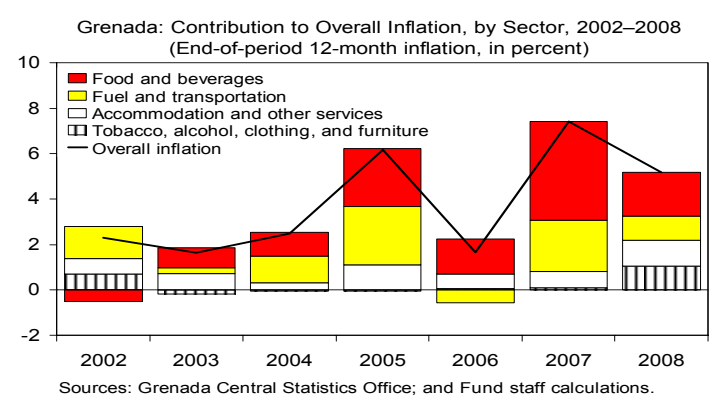

5. The difficulties of the Trinidad and Tobago-based CL Financial Group have increased financial sector uncertainty. The group has a number of linkages to the Grenadian economy, including the presence of two insurance subsidiaries which have been offering deposit-like products to individuals and public and private entities, and majority ownership of Republic Bank Limited. ${ }^{2}$ There is some public concern about delays in payments on policies, and the government has asked policy holders to avoid seeking early surrenders. The exposure of the insurance subsidiaries to policy and deposit holders in the Eastern Caribbean Currency Union (ECCU) region amounts to about EC $\$ 2$ billion (around 15 percent of ECCU GDP). ${ }^{3}$ This development is worrisome in the context of other events which have adversely affected financial sector confidence, including the failure of Capital Bank, an unregulated bank, and of SGL Holdings, an investment scheme promising unusually high returns. Grenada appears not to have any direct exposure to the Stanford Financial Group.

6. The banking system, which is dominated by subsidiaries of international banks, has remained resilient. Private sector credit growth slowed to 9.3 percent at end-February 2009, reflecting weaker economic activity. As of end-December 2008, the ratio of nonperforming loans (NPLs) to total loans remained low at 3.5 percent, and liquidity and capital adequacy ratios were also adequate (Figure 2). However, banks report increases in NPL ratios in the first quarter of 2009, as some nonresident and resident borrowers are experiencing repayment difficulties.

7. The government increased the frequency of adjustments in retail fuel prices from an eight-week to a four-week cycle in order to reduce the magnitude of each adjustment. The government also recently cleared arrears to fuel importers resulting from incomplete passthrough of world fuel prices in 2007-08 through an arrangement under which 50 percent of the fuel tax was remitted back to importers.

\footnotetext{
${ }^{2}$ There was a mini-run on Republic Bank's Carriacou branch after Trinidad and Tobago announced the intervention of CL Financial Group, but the situation quickly stabilized.

${ }^{3}$ See Eastern Caribbean Currency Union-Staff Report for the 2009 Discussion on Common Policies of Member Countries, (IMF Country Report No. 09/175).
} 


\section{B. Outlook}

8. The global economic slowdown has severely weakened Grenada's economic outlook. Real GDP is projected to decline by 0.7 percent in 2009 , lower than the earlier projection of 1.6 percent growth, and the risks are to the downside. ${ }^{4}$ Stayover arrivals are expected to decline further, by about 10 percent, and remittances are likely to slow down sharply. Difficulties in securing financing for major projects will lower FDI inflows markedly, resulting in a further sharp contraction of the construction industry. Annual inflation is projected to fall to 2.1 percent by end-2009 as lower world fuel and food prices are passed through. The current account deficit is expected to narrow sharply, notwithstanding a decline in tourism receipts, reflecting lower fuel and food prices and lower FDI-related imports, while the overall balance is projected to deteriorate significantly. Although the banking sector is expected to remain resilient, there is continuing uncertainty over how developments with CL Financial Group will affect Grenada.

\section{Performance Under the Program}

9. All end-December 2008 quantitative performance criteria were met, reflecting the strong remedial measures put in place in the second half of 2008 . In particular, the authorities met the target on the primary deficit excluding grants, which fell from EC $\$ 88$ million (5.1 percent of GDP) in the first half of 2008 to EC $\$ 41$ million (2.4 percent of GDP) in the second half..$^{5}$ A major effort was made to lower expenditure arrears, which fell from EC\$33 million (1.9 percent of GDP) at end-June 2008, before the change in government, to EC\$9.7 million (0.6 percent of GDP) at end-December 2008. Furthermore, the government was able to roll over late principal payments, which helped to reduce arrears older than 60 days to only EC $\$ 8.4$ million ( 0.5 percent of GDP), meeting this performance criterion by a significant margin.

\section{A major component of the fiscal adjustment was the reduction in capital} expenditure by 3 percentage points of GDP in the second half of the year. This expenditure category accounts for one-third of total spending and has been difficult to control. Despite this adjustment, capital expenditure of EC\$215 million (12.5 percent of GDP) in 2008 was still higher than the programmed EC\$184 million (10.7 percent of GDP). Revenue in the second half of the year exceeded projections by 0.6 percent of GDP, due to the strong performance of income and profits taxes.

\footnotetext{
${ }^{4}$ The sustained growth of the offshore university and the agriculture sector are expected to support output growth, though the weights of those sectors in real GDP are relatively small.

${ }^{5}$ The target is measured below the line; the improvement measured above the line was not as large.
} 


\section{Meeting some} structural benchmarks is taking longer than envisaged. The structural performance criterion on initiating reorganization or liquidation of Capital Bank was met in November 2008, which is a step toward resolving a longstanding financial sector issue. The bank's owner was arrested in March 2009 on charges of fraudulent breach of trust. A Debt Management Unit was established in the Ministry of Finance in January 2009 to improve the effectiveness of and develop a strategy for debt management. The authorities submitted the new VAT Bill to Parliament in April, and plan to submit the Excise Bill in August 2009 rather than in April as previously envisaged. The benchmark on submitting investment incentives legislation ${ }^{6}$ was missed due to the time needed to consult stakeholders, while the benchmark on completing a Country Poverty Assessment (CPA) has been delayed reflecting the time needed to complete the technical work and drafting of the report. These measures are important to improve the business environment and to lay the groundwork for a full PRSP, respectively. A tax holiday was issued to a call center in February $2008 .^{7}$

\section{POLICIES FOR 2009}

\section{The government's economic strategy is focused on coping with the shock to} tourism and FDI while laying the groundwork for broad-based growth. Policy discussions focused on adapting the fiscal framework to reflect the reality of reduced revenue and financing. Staff supported the authorities' planned measures to address the shock, but stressed that fiscal and debt dynamics should be monitored closely to ensure a sustainable trajectory. Other key policy issues discussed were fiscal and institutional reforms, plans to enhance the business environment, and financial sector vulnerabilities.

\section{A. Fiscal Policies and Debt Sustainability}

\section{The outlook for budgetary financing in $\mathbf{2 0 0 9}$ has deteriorated. Divestment} proceeds from a luxury hotel project - the expected main financing source for the 2009 budget — will not materialize this year. The projection for trade-related taxes has been revised downward by 19 percent, which is in line with the shortfall in the first quarter of

\footnotetext{
${ }^{6}$ LEG has provided technical assistance on drafting.

${ }^{7}$ Refraining from issuing further tax holidays was a benchmark for the first review, which was missed.
} 
2009. In addition, PetroCaribe-related grants will also fall sharply reflecting: (i) lower fuel prices; (ii) a lower share of imports financed under the agreement when the diesel price is below US $\$ 100$ per barrel; and (iii) the decision to transfer 35 percent rather than 65 percent of amounts financed to the budget as grants. ${ }^{8}$

\section{The government has adopted a two-pronged strategy to address the shock to tourism and FDI, which emphasizes the need for early intervention and fiscal adjustment:}

- $\quad$ First, within a tighter budget envelope, capital spending programs providing the greatest economic stimulus will be accelerated. The Cabinet has selected a list of priority government and state-owned enterprise projects, taking into account employment generation potential, geographic distribution, and economic visibility. The government will also seek to expedite approvals needed for private sector projects. Reflecting financing constraints, including limited divestment proceeds, the capital spending target has been lowered to EC $\$ 160$ million (8.9 percent of GDP). ${ }^{9}$ The government will also shift from a system of quarterly to monthly allocations to ministries as an expenditure control mechanism to help ensure that the target is met.

- $\quad$ Second, the government will undertake targeted social spending to mitigate the impact of the economic slowdown on the employment and incomes of vulnerable groups. ${ }^{10}$ The public assistance program has been streamlined to remove ineligible individuals, while new qualified persons have been added, and a road maintenance program will provide direct support to poor households. These initiatives are expected to cost EC $\$ 6$ million (0.3 percent of GDP), which is incorporated in the fiscal program.

The mission supported the strategy of accelerating high impact capital spending within a tighter budget envelope, in light of the limited room for maneuver. It also welcomed the

\footnotetext{
${ }^{8}$ Staff had previously recommended that the state-owned company PetroCaribe Grenada Ltd. increase the share of PetroCaribe financing set aside in a special account to pay debt service; the larger share now being set aside will be more than sufficient. The mission noted the need to limit government exposure of the special account.

${ }^{9}$ Based on preliminary data, capital spending in the first quarter slowed sharply to EC $\$ 23$ million or EC\$92 million (5.1 percent of GDP) at an annual rate, reflecting the tight financing situation.

${ }^{10}$ Together with the World Bank, the authorities are developing an aggregate measure of social spending. Preliminary Fund staff calculations indicate that recurrent social spending in 2009 (including transfers to households, education, health, housing, community development, and youth programs) is expected to remain around the same share of GDP as in 2008, while social sector capital project spending will decline, in line with the overall lower capital expenditure as a share of GDP and reflecting the authorities' efforts to improve the efficiency of social spending.
} 
progress in streamlining transfers, and the emphasis on targeted relief to vulnerable groups using the tools available.

\begin{tabular}{|c|c|c|c|c|c|c|c|c|c|}
\hline \multicolumn{10}{|c|}{$\begin{array}{l}\text { Fiscal Developments in 2006-09 } \\
\text { (In percent of GDP) }\end{array}$} \\
\hline & \multirow{3}{*}{$\begin{array}{r}2006 \\
\text { Actual } \\
\end{array}$} & \multirow{3}{*}{$\begin{array}{r}2007 \\
\text { Actual } \\
\end{array}$} & & \multirow{2}{*}{\multicolumn{2}{|c|}{$\begin{array}{r}2008 \\
\text { Second Half }\end{array}$}} & & & & \\
\hline & & & \multirow{2}{*}{$\begin{array}{l}\text { First Half } \\
\text { Prel. }\end{array}$} & & & \multicolumn{2}{|c|}{ Full year } & \multicolumn{2}{|c|}{2009} \\
\hline & & & & Prog. & Prel. & Prog. & Prel. & Prog. & Proj. \\
\hline Total revenue and grants & 33.6 & 27.2 & 15.1 & 15.7 & 16.2 & 30.8 & 31.3 & 30.7 & 26.7 \\
\hline Revenue & 24.9 & 26.1 & 13.5 & 12.8 & 13.4 & 26.3 & 26.9 & 27.0 & 24.2 \\
\hline Grants & 8.7 & 1.1 & 1.6 & 3.0 & 2.7 & 4.5 & 4.3 & 3.6 & 2.5 \\
\hline Total expenditure & 40.0 & 35.3 & 19.8 & 15.3 & 17.2 & 35.1 & 37.0 & 33.0 & 31.6 \\
\hline Current expenditure & 21.2 & 21.7 & 12.0 & 12.4 & 12.5 & 24.4 & 24.5 & 23.0 & 22.7 \\
\hline Of which & & & & & & & & & \\
\hline Interest & 2.1 & 2.3 & 1.2 & 1.5 & 1.4 & 2.7 & 2.6 & 3.1 & 3.0 \\
\hline Capital expenditure & 18.8 & 13.6 & 7.8 & 2.9 & 4.7 & 10.7 & 12.5 & 10.0 & 8.9 \\
\hline Primary balance (excluding grants) $1 /$ & -13.0 & -6.9 & -5.1 & -1.0 & -2.4 & -6.1 & -7.5 & -2.9 & -4.4 \\
\hline Overall balance (including grants) $1 /$ & -6.4 & -8.2 & -4.7 & 0.4 & -1.0 & -4.3 & -5.7 & -2.4 & -4.9 \\
\hline
\end{tabular}

Sources: Ministry of Finance; and Fund staff estimates and projections.

$1 /$ Measured above the line.

\section{In addition, the government is considering providing temporary assistance to} support the tourism sector. ${ }^{11}$ This could include increasing the budget for marketing and airline support by up to EC $\$ 10$ million (0.6 percent of GDP), and the provision of temporary tax relief on hotels' food imports. The mission recommended that the government choose measures that are time bound and have limited fiscal impact. The marketing and airline support, which the authorities classify as capital expenditure, would need to be accommodated by cutting some other projects.

\section{The government recognizes the seriousness of fiscal challenges in 2009 , and is undertaking adjustment measures:}

- $\quad$ On the revenue side: maintaining the specific fuel tax of EC $\$ 3$ per gallon; and enhancing use of tax enforcement measures, including garnishing income and seizing assets, following completion of the tax amnesty at end-April. ${ }^{12}$ The mission noted the potential adverse impact of any further extensions to the tax amnesty on the credibility of the tax system.

\footnotetext{
${ }^{11}$ A recent communique of the Organization of the Eastern Caribbean States (OECS) and the Eastern Caribbean Central Bank (ECCB) Monetary Council supported the introduction of measures to provide short-term relief. Grenada may face pressure to match incentives provided by its regional competitors.

${ }^{12}$ The tax amnesty was extended from end-March to end-April only for taxpayers who had already applied by the end of March. It has yielded EC\$7.5 million, nearly EC\$9 million less than targeted, although the amount collected may increase. The government has also reestablished the Tax Tribunal, a court specialized in hearing tax issues.
} 
- $\quad$ On the expenditure side: freezing wages for public service workers; ${ }^{13}$ targeting capital expenditure as described above; and increasing the efficiency of spending on goods and services, supported by the new Waste Reduction Unit.

\section{These developments have necessitated a revision in the fiscal framework for} 2009. Given the size of the shortfall in revenue, the measures described above will not be sufficient to meet the end-June 2009 target for the primary balance excluding grants. The government has proposed increasing the primary deficit excluding grants target for end-June from EC \$28 million (1.6 percent of GDP) to EC\$53 million (2.9 percent of GDP), while proposing an end-November target of EC $\$ 78$ million (4.3 percent of GDP). ${ }^{14}$ This target would still imply an adjustment in the below-the-line primary deficit of nearly 2 percentage points of GDP from 2008 to 2009. The government plans to maintain domestic arrears older than 60 days close to the low level achieved at the end of 2008.

\section{The government has sought additional budget financing to cover the remaining}

gap after the expenditure reductions. In addition to arranging a syndicated loan from domestic commercial banks, the government has approached the Caribbean Development Bank (CDB) and World Bank for budgetary support loans. ${ }^{15}$ Staff is supporting the authorities' request to raise the concessionality of borrowing from the CDB, and will coordinate with the CDB and World Bank on reform programs attached to the loans.

\section{The debt-to-GDP ratio is not expected to fall below 60 percent by the ECCB's} target date of 2020. Under the revised framework with lower GDP growth and higher debt financing, the debt-to-GDP ratio is not expected to reach 60 percent until 2024. According to the updated Debt Sustainability Analysis (DSA) (Supplement 1), Grenada's debt trajectory has deteriorated since the previous DSA, and Grenada remains at high risk of debt distress. The debt service burden will increase in 2009, reflecting the end of the grace period on obligations to commercial banks restructured in 2005, and the step up in the interest rate from 1 to 2.5 percent on restructured debt to other

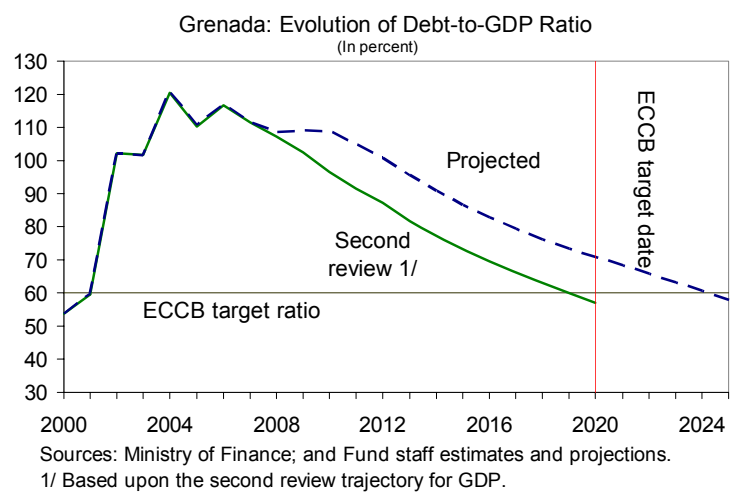
parties.

\footnotetext{
${ }^{13}$ A new public sector wage agreement for 2009-11 had been due for negotiation.

${ }^{14}$ An end-November target for the performance criterion will allow sufficient time to complete the fifth review before the end of the PRGF arrangement on April 16, 2010.

${ }^{15}$ The fiscal framework assumes that during 2009-10, Grenada will receive US\$12 million in budgetary support from the CDB and US\$8 million from the World Bank.
} 


\section{The government is considering a US\$50-80 million (7-12 percent of GDP)} concessional loan from the Export-Import Bank of China to build a luxury hotel in a joint venture, which they may structure as a public-private partnership (PPP). ${ }^{16}$ The authorities believe that government intervention to move this project forward is necessary to stimulate economic activity in the short run and to boost the economy in the long run. The mission noted that such a PPP, which would atypically be primarily government financed, carried operational and commercial risks in an area typically left to the private sector. The mission noted that with an already high debt level (108.6 percent of GDP at end-2008), such a large loan would undermine the authorities' debt reduction objective. The DSA indicates that an US\$80 million loan would increase the present value of the debt-to-GDP ratio by around 5 percentage points in the medium-term (2009-14), even assuming the project led to higher growth. The program's ceiling on bilateral concessional borrowing does not accommodate this loan.

\section{B. Structural Reforms}

21. The government intends to move forward with structural reforms, while sharpening their focus in light of capacity constraints. These reforms are important not only to overcome the economic crisis in the near term but also to lay the basis for long-term sustained growth by improving competitiveness and promoting private sector-led growth. Major areas of structural reforms are tax and customs reform, enhancing the business environment, improving economic management, and laying the groundwork for a comprehensive poverty reduction strategy.

\begin{tabular}{|c|c|c|}
\hline & Target Date & Status \\
\hline $\begin{array}{l}\text { Recruit and begin training staff and adopt } \\
\text { transitional procedures for bonded warehouses }\end{array}$ & May 2009 & Benchmark \\
\hline Complete the Country Poverty Assessment & August 2009 & Proposed Benchmark \\
\hline Submit the new Excise Bill to Parliament & August 2009 & Proposed Benchmark \\
\hline $\begin{array}{l}\text { Develop and begin implementing a customs Fraud } \\
\text { Control Plan }\end{array}$ & August 2009 & Benchmark \\
\hline Establish a Public Procurement Authority & September 2009 & Benchmark \\
\hline Appoint a separate Registrar of Companies & October 2009 & Proposed Benchmark \\
\hline
\end{tabular}

- The mission welcomed the planned introduction of a VAT in February 2010, which is expected to improve the efficiency of the tax system as it has in other ECCU countries. The authorities have finalized the policy issues, and also begun work on public awareness and education. They plan to recruit and begin training staff, and to adopt transitional procedures for bonded warehouses by May 2009. They are drawing on CARTAC technical assistance on implementation. The new VAT and Excise Bills are expected to be passed by Parliament in May and August 2009, respectively. The mission urged the authorities to adhere to the established schedule for VAT implementation.

\footnotetext{
${ }^{16}$ The legal and regulatory framework to facilitate a PPP would need to be enhanced. The government is not actively considering a possible loan to build a marina and port.
} 
- Work toward introducing a market-based property tax now underway will also enhance revenue and the effectiveness of the tax system. The cadastral survey, which will provide the basis for reassessing the value of property, is expected to be completed in the near future. The introduction of new valuations may be delayed until January 2011 to avoid introducing two major tax reforms in the same year.

- The customs Fraud Control Plan, to be developed and implemented by August 2009, is an important part of the customs reform strategy. This plan will lay the basis for risk-based inspections, which together with a new information technology system planned for 2010, will improve customs revenue and efficiency. The plan is part of a broader strategy to enhance the efficiency and effectiveness of the Ports and Customs Authorities, as discussed in a recent national consultation.

- The government is developing an action plan to improve Doing Business Indicators, with technical assistance from the World Bank. Five priority areas have been identified: starting a business, trading across borders, paying taxes, registering property, and enforcement of contracts. Appointment of a separate Registrar of Companies, which is expected to be an important component of a one-stop shop for business start-ups, has been identified as a structural benchmark to be completed by October 2009 for the fourth review.

The Country Poverty Assessment (CPA) will provide essential information to improve targeting of social programs, and to develop a comprehensive poverty reduction strategy. A summary of key findings was submitted to Cabinet in May 2009, and the structural benchmark on completing the CPA has been rephased for August 2009. The authorities will build on this assessment, together with an earlier National Development Strategy, to finalize a full PRSP by November 2009.

- A major reform of the Ministry of Finance is aimed at improving the capacity for economic management. The new Debt Management Unit is already making progress with plans to improve debt monitoring and the capacity to assess debt sustainability. The new Private Sector Development Office has also reinvigorated efforts to improve the business environment. A Waste Reduction Unit has recently been established to reduce wasteful spending. Plans are also underway to create a Division of Economic Management and Planning to sharpen the analytical underpinnings of the policy framework and a Public Procurement Authority to enhance the quality, integrity, and transparency of public procurement.

22. The resolution of Capital Bank will proceed once the High Court has ruled on the legal challenges by the bank's owner. The authorities are appropriately considering options to address the owner's legal challenges. The delay in resolving the bank partly reflects weaknesses in the legal framework and the court's limited experience with such cases. Given the passage of time and the diminution of assets, the mission recommended that the government proceed immediately to liquidate the bank once the legal obstacles are overcome. 
23. The authorities are working to address financial sector uncertainty stemming from the intervention of CL Financial Group. Regional leaders have moved to establish a "Liquidity Support Fund" for British American Insurance, an insurance subsidiary of CL Financial Group, and to coordinate regional information sharing and response planning. ${ }^{17}$ In addition, the Grenada Authority for the Regulation of Financial Institutions (GARFIN) has prohibited the two insurance subsidiaries of CL Financial Group operating in Grenada from offering deposit-like products with high interest rates. Parliament is expected to pass the new Insurance Act by end-June 2009, and its consideration has been expedited in light of the circumstances.

\section{The global and regional financial turmoil have heightened the need to further} strengthen nonbank financial sector regulation and supervision. GARFIN is continuing efforts to develop its capacity, with assistance from CARTAC, and is enhancing legislation, producing regulations, and developing and implementing supervisory practices. It is tightening supervision over credit unions, and has pursued a strategy of consolidation to create a sector with fewer, stronger institutions. The government submitted the Money Services Act to Parliament in March 2009, and plans to submit the Cooperative Societies Act in June 2009.

\section{Program Issues}

\section{A. Program Design}

25. The attached Letter of Intent and accompanying Supplementary Memorandum of Economic Policies outline the authorities' policy objectives for the remainder of 2009. They have proposed relaxing the end-June 2009 performance criterion on the primary balance excluding grants. Quantitative performance criteria for end-June and end-November 2009 and structural measures are shown in Tables 1 and 2 of Attachment II. The authorities expect to complete the benchmark for the third review on submitting investment incentives legislation by end-May. The benchmarks on submitting the new Excise Bill to Parliament and completing the Country Poverty Assessment have been rephased to August 2009.

\footnotetext{
${ }^{17}$ The ECCU members' joint contribution to the Fund would be US $\$ 10$ million. Regional leaders, together with the ECCB, are working out the modalities for this contribution. The program incorporates an adjustor on the primary fiscal balance and net credit targets for potential insurance sector support.
} 
26. The authorities have requested an augmentation (37.5 percent of quota) of the access under the PRGF Arrangement to help mitigate the adverse effects of the tourism and FDI shock. Tourism receipts and FDI are expected to fall by US\$16 million ( 3 percentage points of GDP) and US\$59 million (10 percentage points of GDP), respectively (see text table). Exports and private transfers are also projected to decline. The reduction in imports due to exogenous factors, on account of lower FDI (US\$42 million) and of lower food and fuel prices (US\$16 million), will not fully offset these projected declines, giving rise to a financing gap of US\$11.6 million. To cushion the balance of payments impact, the authorities have requested that an additional SDR 4.39 million (around US\$6.5 million) be disbursed at the conclusion of the third and fourth reviews in equal amounts. ${ }^{18}$ This augmentation would increase access from 102.5 to 140 percent of quota, which would be in line with the new access norm for firsttime PRGF users. ${ }^{19}$
Grenada: Estimated Net Impact of Tourism and FDI Shock

\begin{tabular}{|c|c|c|c|}
\hline & 2008 & 2009 & Difference \\
\hline & \multicolumn{3}{|c|}{ (In millions of U.S. dollars) } \\
\hline Current account & -269.9 & -214.1 & 55.8 \\
\hline \multicolumn{4}{|l|}{ Of which: } \\
\hline (A) Exports & 33.0 & 32.7 & -0.3 \\
\hline (B) Imports & -339.5 & -264.1 & 75.4 \\
\hline \multicolumn{4}{|l|}{ Of which: } \\
\hline (C) FDI-related (both fuel and nonfuel & $\ldots$ & $\ldots$ & 41.8 \\
\hline (D) Fuel and food (price effect) & $\ldots$ & $\ldots$ & 16.1 \\
\hline (E) Endogenous adjustment & $\ldots$ & $\ldots$ & 17.4 \\
\hline (F) Travel (net) & 112.0 & 96.1 & -16.0 \\
\hline Of which: Receipts & 128.6 & 112.1 & -16.5 \\
\hline (G) Private transfers & 19.2 & 17.4 & -1.9 \\
\hline Capital and financial account $1 /$ & 257.9 & 182.7 & -75.3 \\
\hline (H) Of which: Direct investment (net) & 161.2 & 102.3 & -58.9 \\
\hline Overall balance & -12.0 & -31.5 & \\
\hline Financing & 12.0 & 19.8 & \\
\hline \multicolumn{4}{|l|}{ Of which: } \\
\hline Change in imputed reserves & 6.4 & 10.3 & \\
\hline Requested augmentation & $\ldots$ & 6.5 & \\
\hline (I) Gross impact $(A+F+G+H)$ & $\ldots$ & $\ldots$ & -77.0 \\
\hline (J) Net impact $(I+C+D)$ & $\cdots$ & $\cdots$ & -19.1 \\
\hline Memorandum items: & \multicolumn{3}{|c|}{ (In percent of GDP) } \\
\hline \multicolumn{4}{|l|}{ Central government operations } \\
\hline Total revenue and grants & 31.3 & 26.7 & -4.5 \\
\hline Of which: Current revenue & 26.9 & 24.2 & -2.7 \\
\hline Total expenditure & 37.0 & 31.6 & -5.4 \\
\hline Overall balance & -5.7 & -4.9 & 0.9 \\
\hline \multicolumn{4}{|l|}{ Financing } \\
\hline \multicolumn{4}{|l|}{ Of which: } \\
\hline Net external financing & 2.6 & 2.3 & \\
\hline Net domestic financing & -0.2 & 1.2 & \\
\hline Requested augmentation & $\ldots$ & 1.0 & \\
\hline
\end{tabular}

Source: Grenada authorities; and Fund staff estimates. $1 /$ Includes net errors and omissions.

\section{B. Program Risks and Financing Assurances}

\section{The program faces significant risks, notwithstanding the authorities'}

demonstrated policy commitment. A more pronounced and prolonged global slowdown could further reduce tourism demand and FDI inflows, which would deepen the economic downturn and lower revenue; lower donor grants and increased financial sector vulnerability are additional risks. The program incorporates adjustors on grants and on insurance sector support, as well as a more focused structural agenda. The authorities are committed to taking contingent measures if needed to reduce spending, including limiting transfers. The possible US\$50-80 million (7-12 percent of GDP) concessional loan from the Export-Import Bank of China, which is now being considered, has not been accommodated under the program's ceiling on the contracting and guaranteeing of bilateral concessional external debt.

\footnotetext{
${ }^{18}$ The remainder of the gap would be filled by possible reschedulings from the Paris Club and Taiwan Province of China.

${ }^{19}$ See Press Release No. 09/138.
} 
28. The authorities have approached the Paris Club for an extension of the 2006-08 debt rescheduling treatment to include 2009. An extension would be useful in the tight external financing environment. The authorities are continuing to seek a settlement with the Export-Import Bank of Taiwan Province of China on terms comparable to that provided by the Paris Club. The authorities are also continuing to make best efforts to conclude the remaining bilateral Paris Club agreement with the Russian Federation and to offer those nonparticipating creditors in the 2005 commercial debt exchange who come forward the same terms as received by other participants in the exchange. The authorities have cleared all but EC $\$ 1$ million in arrears on unrestructured domestic debt to the nonbank public incurred in June 2007, while there are EC $\$ 1.7$ million arrears on 90-day treasury bills held by domestic commercial banks.

\section{Staff Appraisal}

29. Grenada, like the rest of ECCU and the Caribbean, has been hit hard by negative spillovers from the global economic downturn. Its financial system has come under pressure, primarily through the nonbank sector. The very high debt levels in the context of the regional currency board arrangement greatly constrain policy responses to the downturn.

30. The government is approaching these challenges with a strong resolve. After the strong fiscal adjustment in the second half of 2008 and progress with structural reforms, Grenada is now better placed to weather the difficult period ahead. The government is applying the same determination in 2009 in its response to the shock to tourism and FDI buffeting the country.

31. Staff welcomes the government's steps to address the worsened circumstances.

The government is reducing capital spending, imposing a wage freeze, and increasing the efficiency of spending on goods and services in response to significant revenue shortfalls. At the same time, it is seeking to get the greatest economic stimulus possible within the tighter budget envelope by accelerating a list of priority projects. The fiscal framework accommodates additional targeted transfers to assist vulnerable segments of the population. In light of the risks to these groups, staff has underscored the need to complete the Country Poverty Assessment without further delay.

32. The ambitious program of fiscal reform is moving forward. Introduction of a VAT will enhance the coverage and buoyancy of the tax system. It will be important to continue with the thorough approach being taken with preparations, and passage of new VAT and Excise Bills will be a crucial step. Progress with the cadastral survey is laying the basis for a market-based property tax, which has appropriately been delayed until January 2011 to avoid taking on two major tax reforms in the same year.

\section{Improving economic management through institutional reform at the}

Ministry of Finance is a welcome priority. Notable progress has already been made by the new Debt Management Unit and Private Sector Development Office. The creation of a Waste Reduction Unit, a Division of Economic Management and Planning, and the planned Public Procurement Authority are also welcome innovations. 


\section{Caution is warranted regarding the possible loan from the Export-Import} Bank of China to build a luxury hotel. While acknowledging the authorities' desire to stimulate the economy, staff has cautioned that a loan of this magnitude would undermine progress toward debt sustainability and entails significant implementation risks.

\section{Improving tax enforcement and detection powers is essential following} completion of the tax amnesty. The government decided to extend the tax amnesty to end-April only for those who had already approached the government by end-March but needed more time to complete the administrative process. Staff welcomes the tax administration's plans to employ their capacity to garnish income and to seize assets.

36. The authorities need to carefully monitor financial sector vulnerabilities. The banking sector has remained resilient. However, concerns regarding CL Financial Group, which follow upon past difficulties of Capital Bank and an unregulated investment scheme, could pose a risk to confidence in the financial system. Staff welcomes the close cooperation among regional governments to address the troubled CL Financial Group. The government's decisions to revoke Capital Bank's license and to petition the High Court for liquidation are laudable, and the government should proceed directly to liquidation once the court permits. GARFIN needs to continue improving its capacity for nonbank financial supervision and regulation.

37. The deteriorating environment has heightened program risks. Key risks include the potential of a deeper and more prolonged global downturn, uncertainty regarding CL Financial Group, and the possible large loan from the Export-Import Bank of China. The government has responded prudently thus far to the first risk by prioritizing spending plans, enhancing efforts to protect vulnerable groups, and identifying contingent measures. The program's design seeks to address risks, through the identification of contingent fiscal measures, the ceiling on bilateral concessional debt, and adjustors on grants and on insurance sector support.

38. Notwithstanding these risks, staff supports the authorities' requests for completion of the third review and financing assurances review, for modification of a quantitative performance criterion, and for augmentation. The government has shown an impressive commitment to its program. Staff supports the government's efforts, while underscoring the need for perseverance and caution as pressures intensify. 
Figure 1. Grenada: Inflation Developments

Inflationary pressures are receding...

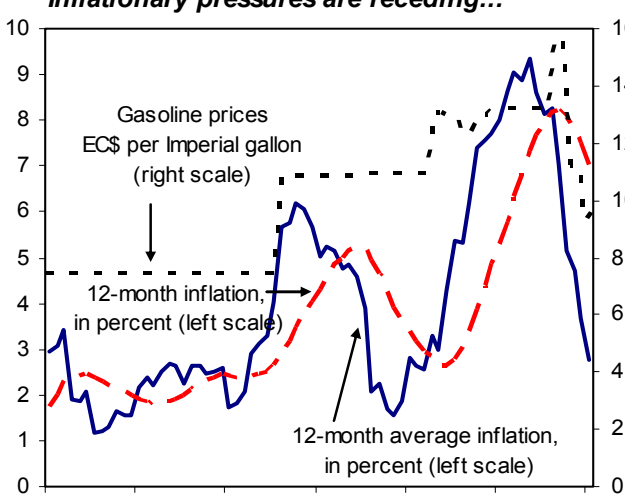

Mar-03 Mar-04 Mar-05 Mar-06 Mar-07 Mar-08 Mar-09

... reducing imported inflation.

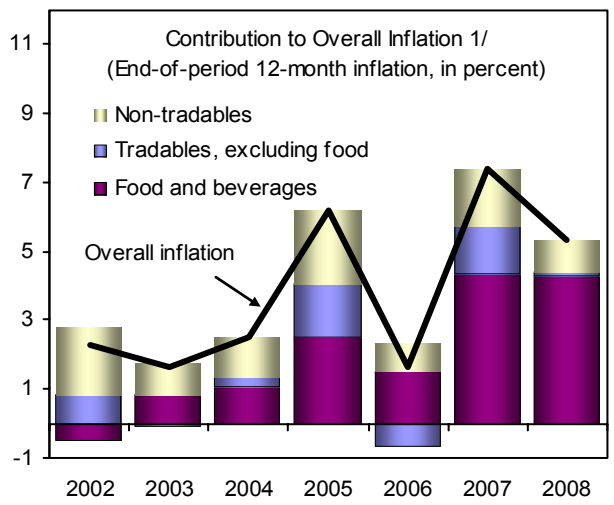

...and declining prices of flour products have contributed to lower inflation.

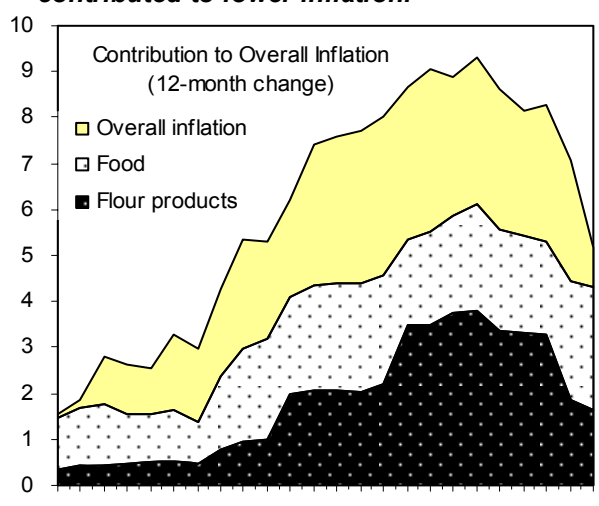

Jan-07 May-07 Sep-07 Jan-08 May-08 Sep-08
... as energy and food prices have fallen back...

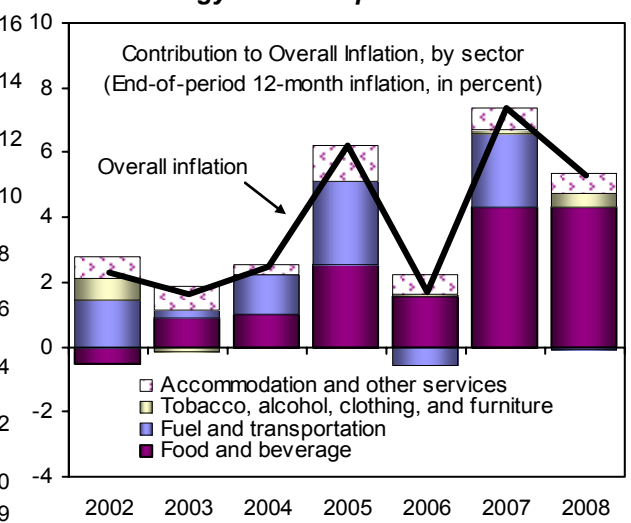

Declining world fuel prices have been passed through...

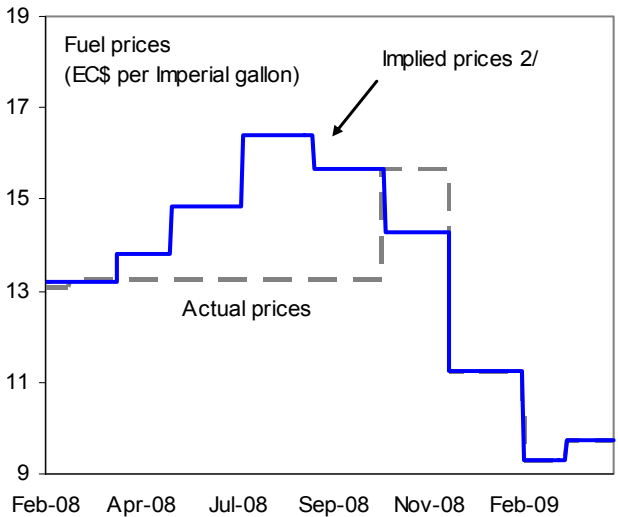

Declining world prices will exert further downward pressure in 2009.

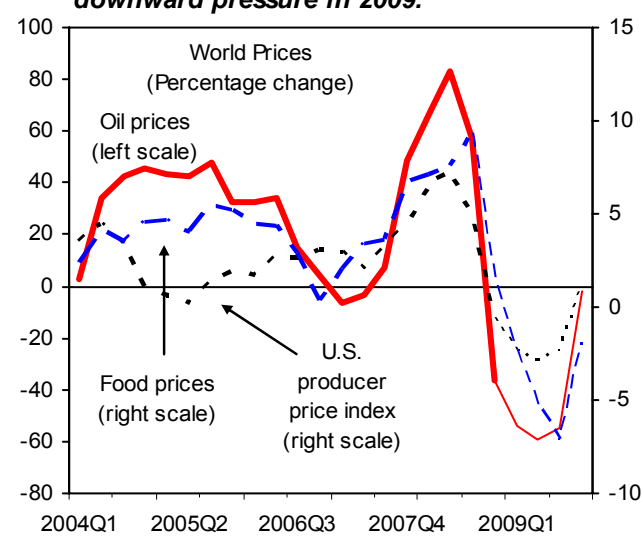

Sources: Grenada authorities; Bloomberg; ECCU; IMF, International Financial Statistics; IMF, World Economic Outlook; and Fund staff calculations.

$1 /$ Tradables comprise food, alcoholic drinks and tobacco, fuel and light, clothing and footwear, household and furniture equipment. Nontradables include medical care and expenses, education, personal services, housing and utilities, and transportation and communication.

2/ Prices implied by the automatic pricing mechanism and assuming the EC $\$ 3$ per gallon specific fuel tax was in place. 
Figure 2. Grenada: Banking System Vulnerabilities

Though liquidity conditions have tightened...

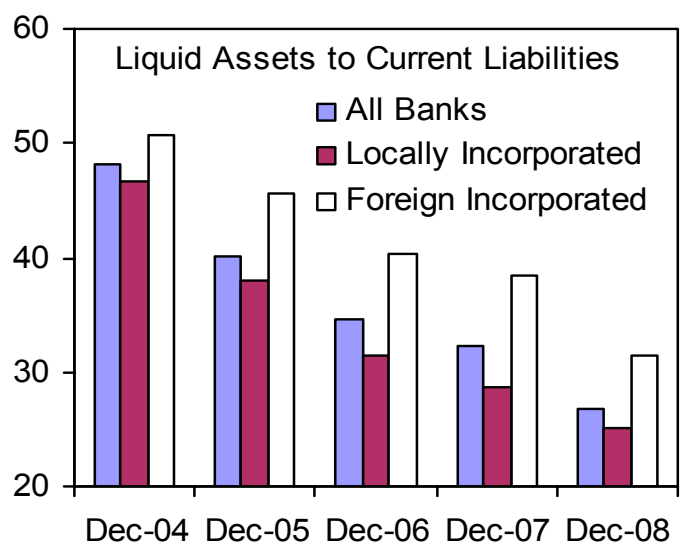

... and NPL ratios have remained at moderate levels...

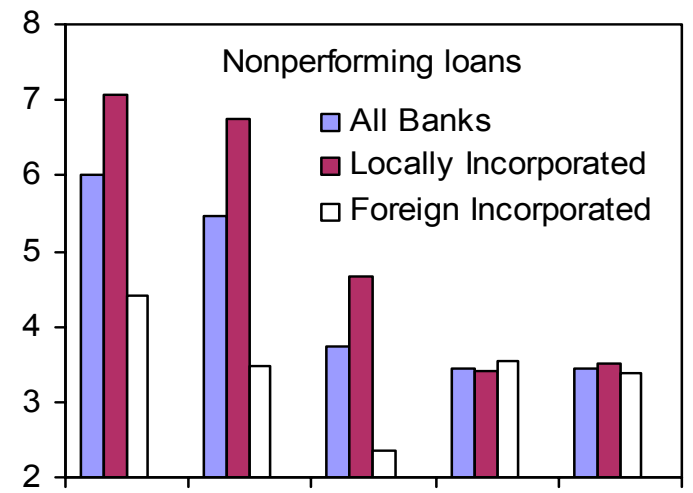

Dec-04 Dec-05 Dec-06 Dec-07 Dec-08

Capital cushions in local banks remained solid, despite their recent decline...

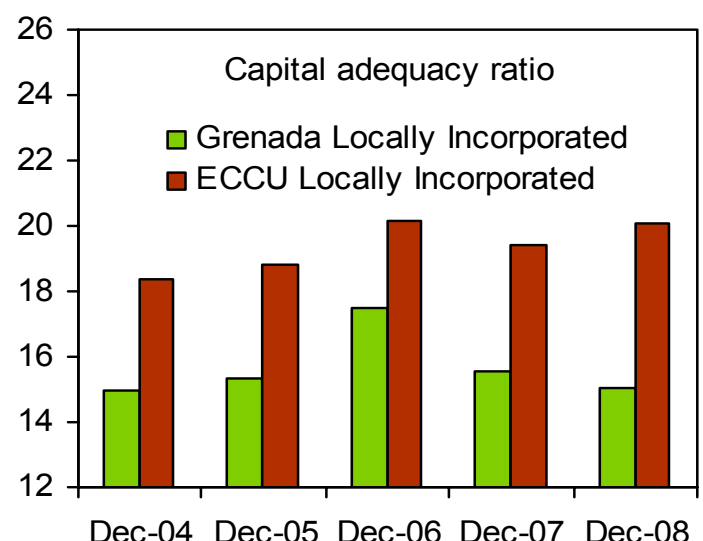

...banks have continued shifting their assets toward loans...

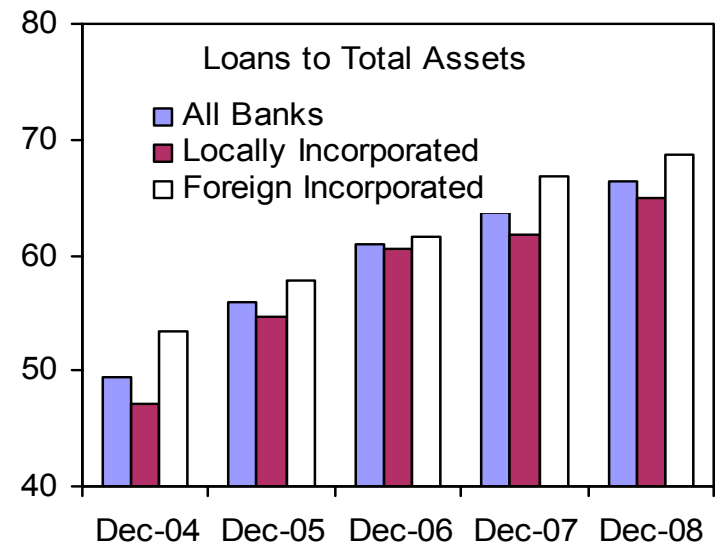

...but the declining trend in provisioning points to the need for vigilance in the banking sector.

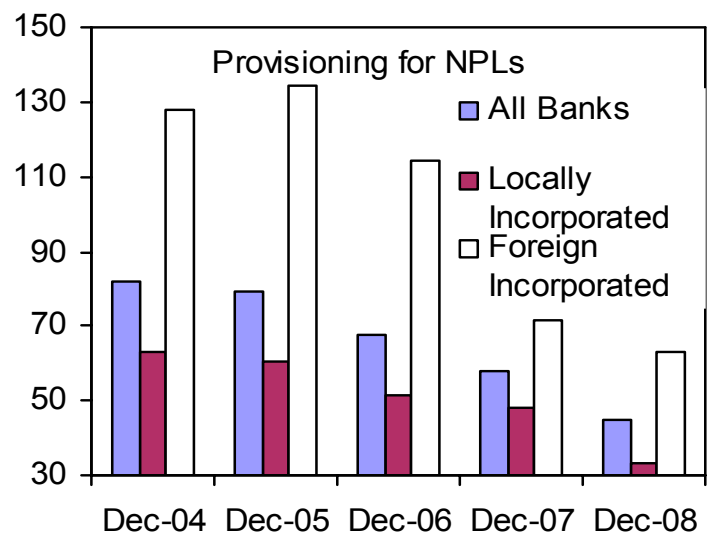

...while bank profitability has remained stable.

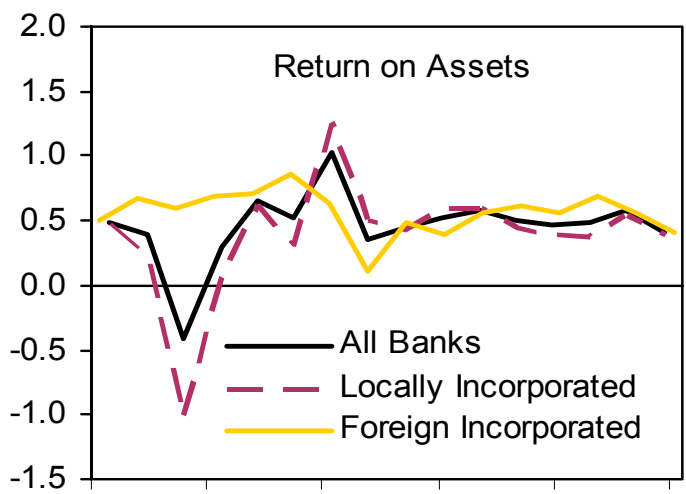

Mar-05 Dec-05 Sep-06 Jun-07 Mar-08 Dec-08

Sources: ECCB; and Fund staff calculations. 
Figure 3. Grenada: Fiscal Sector Indicators (In percent of GDP)

The end-2008 target for the primary balance excluding grants was met...

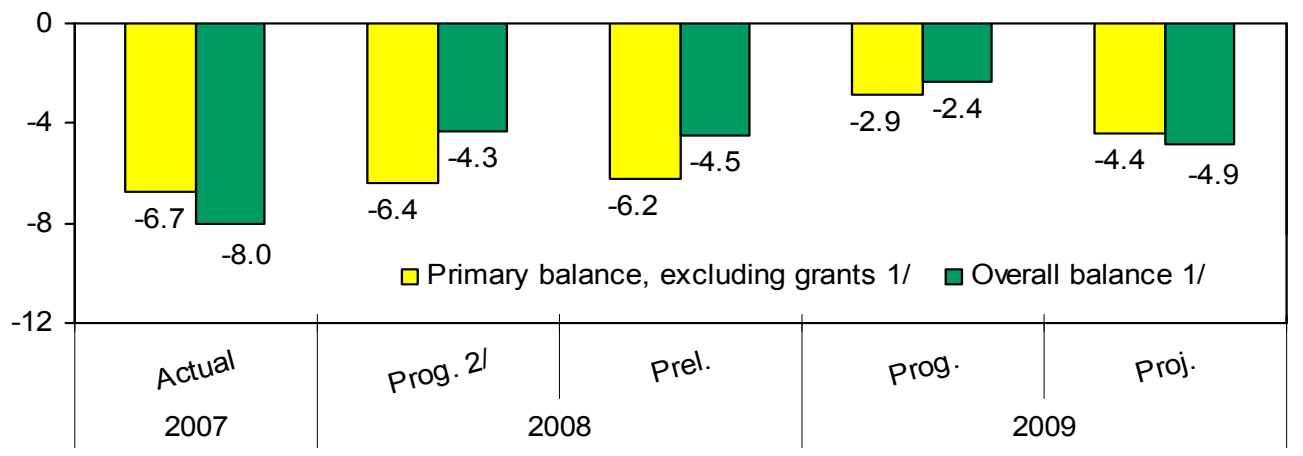

... reflecting better control of capital expenditure in the second half ...

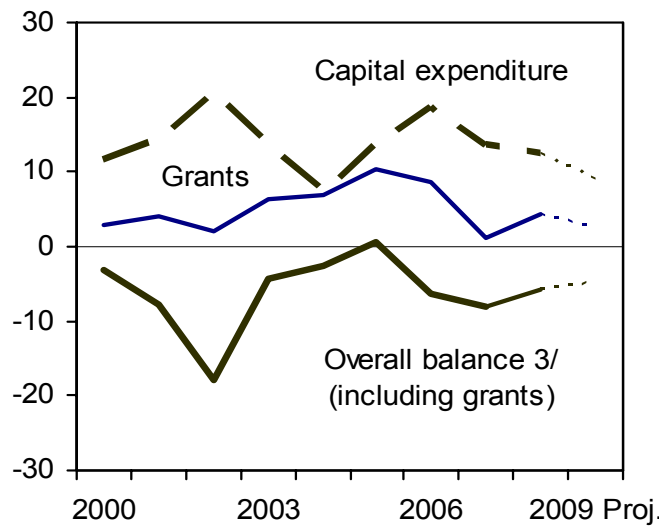

Although the 2006-08 wage agreement implied large unbudgeted retroactive wage increases during 2008...

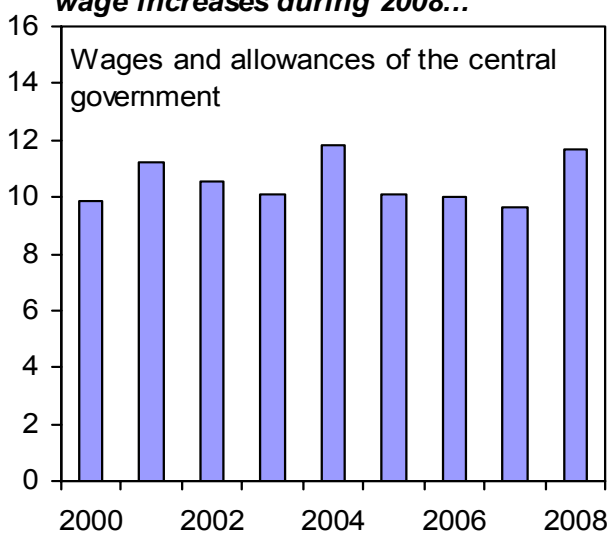

Sources: Grenada authorities; ECCU country authorities; and Fund staff estimates.

1/ Measured below the line.

2/ The target for the primary balance excluding grants is adjusted based on the outturn for grants, concessional loans, and bank restructuring costs.

3/ Includes 11.4 percent of GDP paid in 2002 to extinguish lease arrangements.
... and a strong revenue outturn.

However, the outlook has deteriorated.

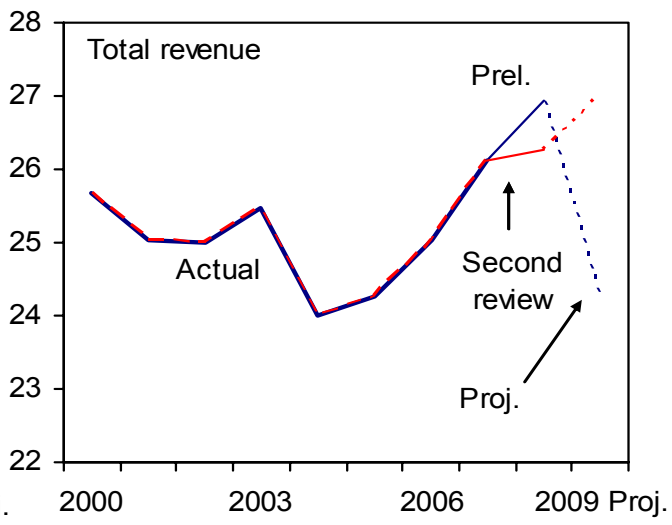

...Grenada's wage bill remains low by regional standards.
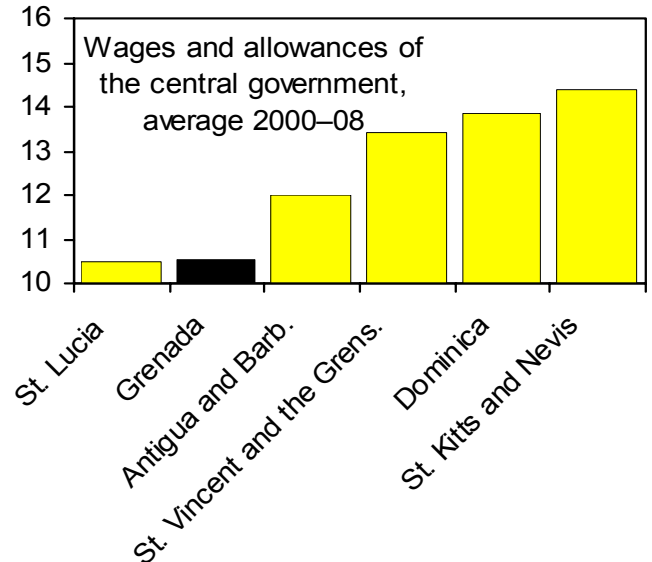
Figure 4. Grenada: Competitiveness Indicators

Declining tourism receipts and exports in 2008, which were partially offset by increasing grants...

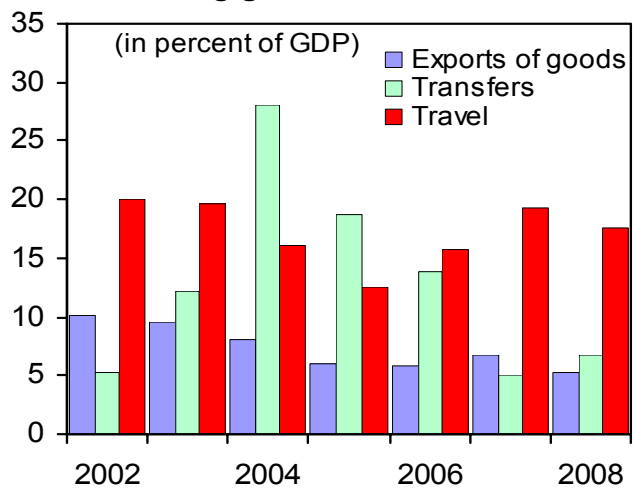

Competitiveness measured by the CPIbased REER appears not to have been a problem...

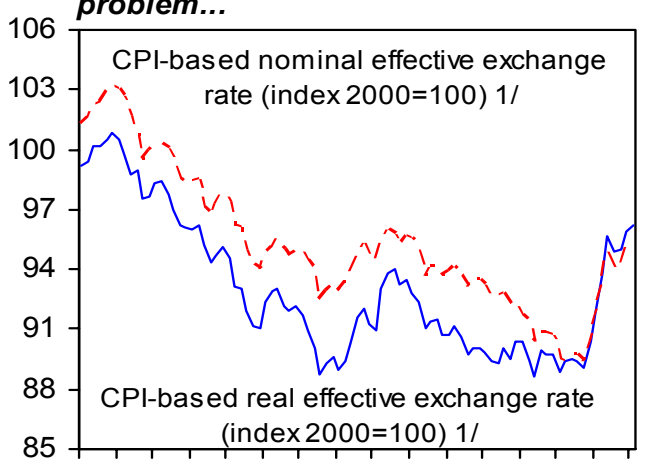

Sep-01 Mar-03 Sep-04 Mar-06 Sep-07 Mar-09 ...led to a slight increase in the current account deficit.

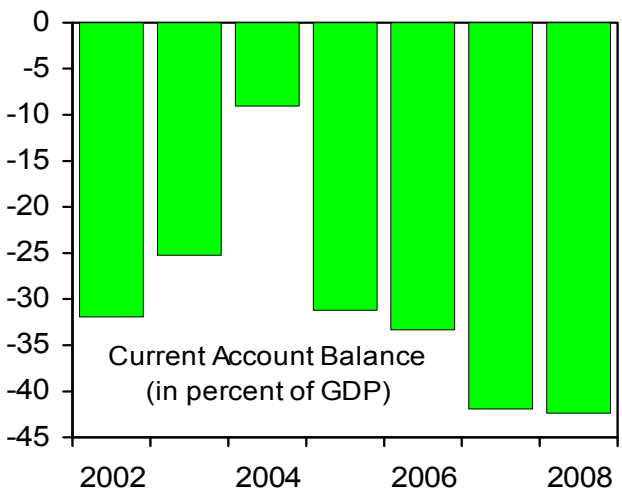

...and competitor- and customer-based measures have been relatively stable.

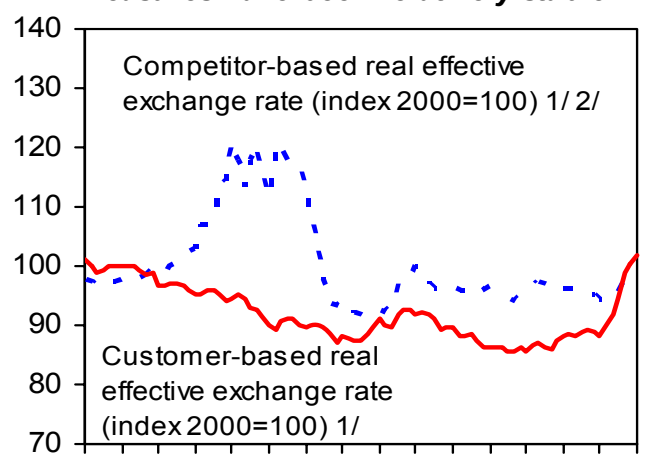

\section{Grenada's tourism share recovered} after the hurricanes but began to decline in 2008...
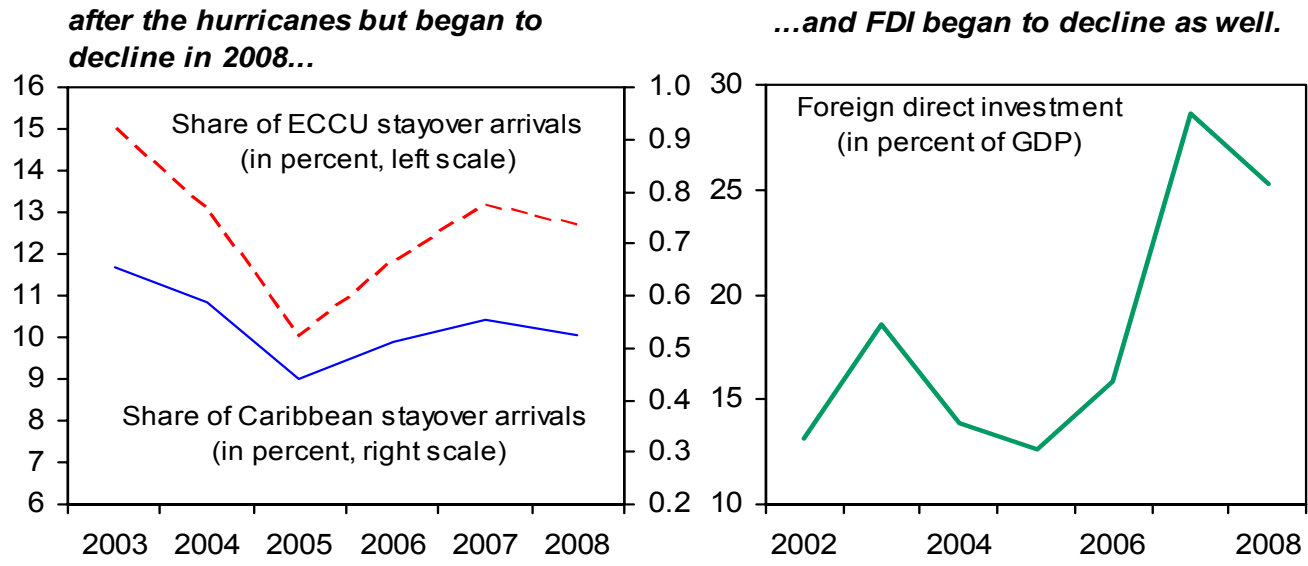

Sources: Grenada authorities; ECCB; Caribbean Tourist Organization; and Fund staff calculations. $1 /$ An increase (decrease) indicates an appreciation (depreciation).

2/ The sharp movements in the competitor-based real exchange rate in 2002-04 were largely driven by the Dominican Republic's peso. 
Figure 5: Grenada: Doing Business Indicators, 2008 1/

The authorities are seeking to improve

doing business indicators...

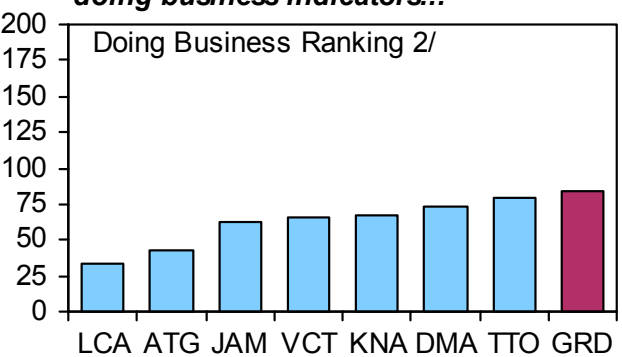

...facilitating payment of taxes..

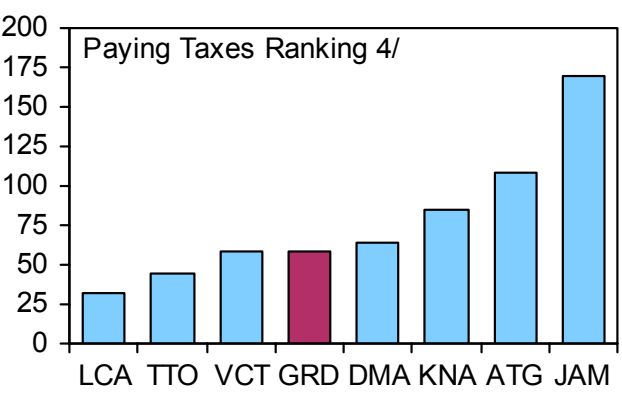

...reducing the obstacles to trade...

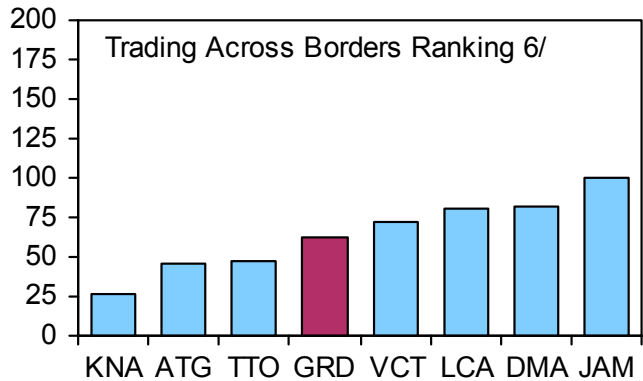

...by making the process of property registration more efficient...

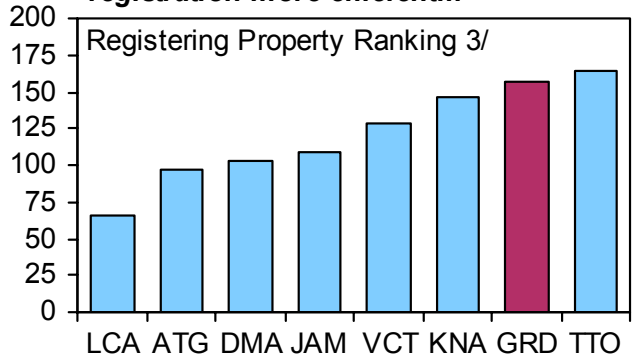

...facilitating commercial contract enforcement.

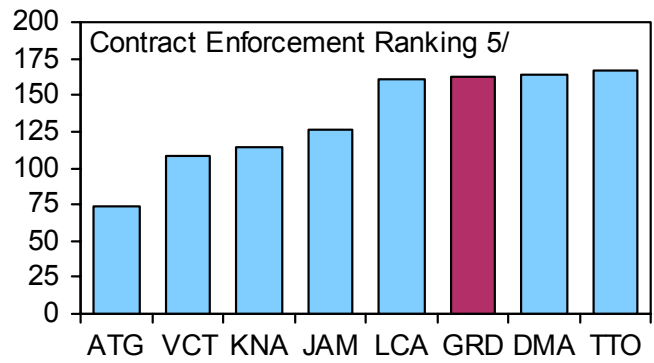

... and removing cumbersome procedures in starting a business.

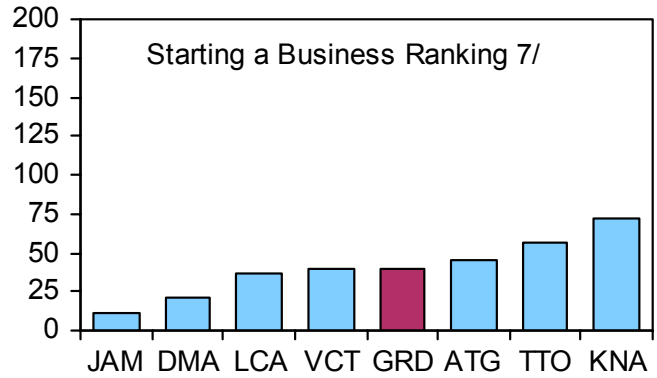

Source: World Bank, Doing Business Indicators (2009).

Note: Antigua and Barbuda (ATG), Dominica (DMA), Grenada (GRD), Jamaica (JAM), St. Kitts and Nevis (KNA), St. Lucia (LCA), Trinidad and Tobago (TTO) and St. Vincent and the Grenadines (VCT).

$1 /$ Smaller numbers represent greater ease in doing business. The indicators are comparable across 181 countries covering the period June 2007 to June 2008.

2/ An overall indicator that captures the regulatory costs of doing business.

$3 /$ Measures the steps, time, and cost involved in registering property.

4/ Measures the tax payments for a manufacturing company in a year, time required to comply with profit taxes, consumption taxes and labor taxes, and total tax rate.

$5 /$ Measures the efficiency of contract enforcement by following the evolution of a sale of goods dispute and tracking the time, cost, and number of procedures involved from the moment the plaintiff files the lawsuit until actual payment.

6/ Measures the procedural requirements, time, and cost involved for trading a standard shipment of goods by ocean transport for a medium-sized domestically-owned private firm with 60 employees located in the economy's most populous city.

7/ Measures the procedures, time, and cost involved in launching a commercial or industrial firm with up to 50 employees and start-up capital of 10 times the economy's per capita gross national income. 
Table 1. Grenada: Selected Economic and Financial Indicators, 2006-10

Rank in UNDP Human Development Index out of 179 countries (2008)

Life expectancy at birth in years (2006) GDP per capita in US\$ (2007)
86

68 5,737
Infant mortality rate per '000 births (2006)

Adult illiteracy rate in percent (2004)

Poverty headcount index (2000)
16

\begin{tabular}{|c|c|c|c|c|c|c|c|}
\hline & & Est. & Prog. 1/ & Prel. & Prog. 1/ & Proj. & Proj. \\
\hline & 2006 & 2007 & \multicolumn{2}{|c|}{2008} & \multicolumn{2}{|c|}{2009} & 2010 \\
\hline & \multicolumn{7}{|c|}{ (Annual percentage change; unless otherwise specified) } \\
\hline \multicolumn{8}{|l|}{ National income and prices } \\
\hline Real GDP & -2.3 & 4.5 & 1.6 & 0.3 & 1.6 & -0.7 & 1.0 \\
\hline GDP deflator & 3.9 & 3.4 & 6.2 & 4.7 & 7.3 & 5.5 & 2.8 \\
\hline \multicolumn{8}{|l|}{ Consumer prices } \\
\hline End-of-year & 1.7 & 7.4 & 6.2 & 5.2 & 3.2 & 2.1 & 2.2 \\
\hline Period average & 4.2 & 3.9 & 7.8 & 8.0 & 4.0 & 2.3 & 2.9 \\
\hline \multicolumn{8}{|l|}{ External sector } \\
\hline Exports of goods & -1.7 & 26.0 & -18.4 & -19.0 & 2.6 & -0.8 & 9.5 \\
\hline Imports of goods & -0.8 & 10.6 & 3.0 & 3.5 & -0.3 & -22.2 & 4.2 \\
\hline Merchandise export volume 2/ & -14.7 & 25.7 & -16.7 & -6.6 & -1.4 & -13.4 & 1.7 \\
\hline Merchandise import volume 2/ & -10.7 & 3.1 & -11.8 & 0.0 & 11.7 & -9.3 & 4.6 \\
\hline Current account balance (including grants; in percent of GDP) & -33.4 & -41.9 & -31.8 & -42.3 & -30.5 & -32.0 & -30.3 \\
\hline FDI (net, in percent of GDP) & 15.9 & 28.7 & 19.7 & 25.2 & 23.1 & 15.3 & 16.7 \\
\hline Real effective exchange rate (end of period, depreciation -) & -4.6 & 0.2 & $\ldots$ & 6.6 & $\ldots$ & $\ldots$ & $\ldots$ \\
\hline \multicolumn{8}{|l|}{ Banking system } \\
\hline Net foreign assets $3 /$ & -7.5 & -1.1 & -1.1 & -9.5 & 1.3 & -3.6 & -3.3 \\
\hline $\begin{array}{l}\text { Net domestic assets } 3 / \\
\text { Of which }\end{array}$ & 8.4 & 12.0 & 7.2 & 13.2 & 7.7 & 4.6 & 5.5 \\
\hline Credit to public sector (net) $3 /$ & -0.3 & 1.6 & -2.0 & 1.0 & -0.1 & 1.9 & 0.0 \\
\hline Credit to private sector $3 /$ & 9.2 & 12.5 & 5.5 & 9.3 & 7.8 & 2.7 & 5.4 \\
\hline Money and quasi-money (M2) & 0.9 & 11.0 & 6.1 & 3.7 & 9.0 & 1.0 & 2.2 \\
\hline Weighted average deposit rate (in percent) & 3.0 & 3.0 & $\ldots$ & 3.2 & $\ldots$ & $\ldots$ & $\ldots$ \\
\hline \multirow[t]{2}{*}{ Weighted average lending rate (in percent) } & 9.7 & 9.6 & $\ldots$ & 9.2 & $\ldots$ & $\ldots$ & $\ldots$ \\
\hline & \multicolumn{7}{|c|}{ (In percent of GDP) } \\
\hline \multicolumn{8}{|l|}{ Central government finances 4 / } \\
\hline $\begin{array}{l}\text { Total revenue and grants } \\
\text { Of which }\end{array}$ & 33.6 & 27.2 & 30.8 & 31.3 & 30.7 & 26.7 & 28.4 \\
\hline Grants & 8.7 & 1.1 & 4.5 & 4.3 & 3.6 & 2.5 & 2.9 \\
\hline Total expenditure & 40.0 & 35.3 & 35.1 & 37.0 & 33.0 & 31.6 & 31.2 \\
\hline Current expenditure & 21.2 & 21.7 & 24.4 & 24.5 & 23.0 & 22.7 & 21.5 \\
\hline \multicolumn{8}{|l|}{ Of which } \\
\hline Salaries and allowances & 10.0 & 9.7 & 11.6 & 11.6 & 10.7 & 10.5 & 10.4 \\
\hline Capital expenditure & 18.8 & 13.6 & 10.7 & 12.5 & 10.0 & 8.9 & 9.7 \\
\hline Primary balance (excluding grants) & -13.0 & -6.9 & -6.1 & -7.5 & -2.9 & -4.4 & -2.8 \\
\hline Primary balance (including grants) & -4.3 & -5.8 & -1.6 & -3.2 & 0.8 & -1.9 & 0.2 \\
\hline Current balance & 3.7 & 4.4 & 1.8 & 2.4 & 4.0 & 1.5 & 4.0 \\
\hline Overall balance (including grants) & -6.4 & -8.2 & -4.3 & -5.7 & -2.4 & -4.9 & -2.7 \\
\hline \multirow[t]{2}{*}{ Public and publicly guaranteed debt (end-period) } & 116.7 & 111.5 & 110.1 & 108.6 & 105.1 & 109.4 & 108.9 \\
\hline & \multicolumn{7}{|c|}{ (In millions of U.S. dollars) } \\
\hline Nominal GDP & 564.4 & 607.9 & 638.5 & 638.5 & 668.8 & 668.8 & 694.4 \\
\hline
\end{tabular}

Sources: Ministry of Finance; Eastern Caribbean Central Bank; United Nations, Human Development Report 2008; World Bank, WDI 2007; and Fund staff estimates and projections.

1/ Corresponds to the staff supplement in IMF Country Report No. 09/95 with all ratios expressed relative to the revised GDP.

2/ Does not include goods procured in ports by carriers.

3/ As a percent of broad money at the beginning of the year.

4/ Measured using above-the-line information. 
Table 2. Grenada: Central Government Finances, 2006-09 (In millions of Eastern Caribbean dollars)

\begin{tabular}{|c|c|c|c|c|c|c|c|c|c|}
\hline \multirow{4}{*}{ Total revenue and grants } & \multirow{4}{*}{$\begin{array}{r}\text { Actual } \\
2006 \\
\mathbf{5 1 1 . 9}\end{array}$} & \multirow{4}{*}{$\begin{array}{c}\text { Actual } \\
2007 \\
445.8\end{array}$} & \multicolumn{5}{|c|}{2008} & \multirow[b]{2}{*}{ Prog. 1/ } & \multirow{3}{*}{ Proj. } \\
\hline & & & \multirow{3}{*}{$\begin{array}{r}\begin{array}{r}\text { Prel. } \\
\text { First Half }\end{array} \\
260.0\end{array}$} & Prog. $1 /$ & Prel. & Prog. 1/ & Prel. & & \\
\hline & & & & \multicolumn{2}{|c|}{ Second Half } & \multicolumn{2}{|c|}{ Full Year } & 2009 & \\
\hline & & & & 271.2 & 278.8 & 531.2 & 538.8 & 553.6 & 482.6 \\
\hline Total revenue & 379.7 & 428.5 & 233.0 & 220.0 & 231.6 & 453.0 & 464.5 & 488.1 & 437.1 \\
\hline Current revenue & 379.6 & 428.4 & 232.9 & 220.0 & 231.5 & 452.9 & 464.4 & 488.0 & 437.0 \\
\hline Tax revenue 2/ & 353.5 & 402.8 & 212.6 & 203.2 & 221.1 & 415.9 & 433.8 & 455.1 & 405.6 \\
\hline Taxes on income and profits & 56.0 & 74.8 & 40.5 & 41.4 & 54.1 & 81.8 & 94.6 & 81.8 & 81.7 \\
\hline Taxes on property & 22.6 & 29.0 & 17.8 & 7.8 & 8.1 & 25.7 & 26.0 & 27.8 & 30.3 \\
\hline Taxes on domestic goods and services & 69.0 & 71.3 & 42.4 & 32.7 & 36.3 & 75.0 & 78.7 & 85.8 & 82.4 \\
\hline Taxes on international transactions & 205.9 & 227.7 & 112.0 & 121.4 & 122.6 & 233.3 & 234.6 & 259.7 & 211.2 \\
\hline Nontax revenue & 26.0 & 25.6 & 20.3 & 16.8 & 10.4 & 37.0 & 30.6 & 32.9 & 31.4 \\
\hline Capital revenue & 0.1 & 0.1 & 0.1 & 0.0 & 0.0 & 0.1 & 0.1 & 0.1 & 0.1 \\
\hline Grants 3/ & 132.2 & 17.3 & 27.0 & 51.2 & 47.3 & 78.2 & 74.3 & 65.5 & 45.5 \\
\hline Total expenditure & 608.9 & 579.7 & 341.1 & 264.0 & 296.7 & 605.0 & 637.7 & 596.1 & 570.5 \\
\hline Current expenditure & 323.1 & 356.6 & 206.8 & 214.3 & 216.1 & 421.0 & 422.8 & 416.1 & 410.3 \\
\hline Current primary expenditure & 291.6 & 318.5 & 186.7 & 188.0 & 192.0 & 374.7 & 378.7 & 360.0 & 355.9 \\
\hline Salaries and allowances & 152.5 & 158.4 & 97.4 & 101.9 & 103.4 & 199.3 & 200.8 & 193.6 & 189.8 \\
\hline Wages and salaries & 135.3 & 140.7 & 86.9 & 90.7 & 93.0 & 177.6 & 180.0 & 166.6 & 162.8 \\
\hline Personnel allowances & 17.1 & 17.7 & 10.5 & 11.2 & 10.3 & 21.7 & 20.8 & 27.0 & 27.0 \\
\hline Goods and services & 71.1 & 79.1 & 37.4 & 37.6 & 47.2 & 75.0 & 84.7 & 77.2 & 73.5 \\
\hline Interest 4/ & 31.6 & 38.1 & 20.1 & 26.3 & 24.1 & 46.3 & 44.2 & 56.1 & 54.4 \\
\hline Domestic & 9.9 & 13.0 & 5.1 & 13.3 & 12.0 & 18.4 & 17.1 & 20.4 & 18.7 \\
\hline Foreign & 21.6 & 25.1 & 15.0 & 13.0 & 12.1 & 27.9 & 27.1 & 35.7 & 35.7 \\
\hline Transfers and subsidies & 68.0 & 81.0 & 51.9 & 48.5 & 41.3 & 100.4 & 93.2 & 89.2 & 92.6 \\
\hline Capital expenditure & 285.8 & 223.1 & 134.3 & 49.7 & 80.6 & 184.0 & 214.9 & 180.0 & 160.2 \\
\hline Current balance & 56.5 & 71.8 & 26.1 & 5.8 & 15.5 & 31.9 & 41.6 & 71.9 & 26.6 \\
\hline Primary balance (excluding grants) & -197.6 & -113.1 & -88.1 & -17.6 & -41.0 & -105.7 & -129.1 & -51.9 & -79.0 \\
\hline Primary balance (including grants) & -65.4 & -95.9 & -61.0 & 33.5 & 6.3 & -27.5 & -54.8 & 13.6 & -33.5 \\
\hline Overall balance (excluding grants) & -229.2 & -151.2 & -108.1 & -43.9 & -65.1 & -152.0 & -173.2 & -108.0 & -133.5 \\
\hline Overall balance (including grants) & -97.0 & -133.9 & -81.1 & 7.3 & -17.8 & -73.8 & -98.9 & -42.5 & -87.9 \\
\hline Statistical discrepancy & -20.8 & 2.6 & 24.8 & -24.8 & -3.2 & 0.0 & 21.6 & 0.0 & 0.0 \\
\hline Financing & 124.7 & 131.3 & 56.2 & 17.6 & 21.0 & 73.8 & 77.3 & 42.5 & 87.9 \\
\hline Net external financing & 54.5 & 40.2 & 3.5 & 42.1 & 40.6 & 45.6 & 44.1 & 11.3 & 58.4 \\
\hline Net amortization & 54.8 & 40.5 & 2.3 & 42.1 & 37.8 & 44.4 & 40.1 & 11.3 & 58.4 \\
\hline Disbursements & 73.8 & 62.7 & 18.1 & 52.7 & 47.5 & 70.9 & 65.7 & 38.7 & 85.8 \\
\hline Amortization & -19.0 & -22.2 & -15.9 & -10.6 & -9.7 & -26.5 & -25.6 & -27.4 & -27.4 \\
\hline Change in government assets & -0.3 & -0.3 & 1.2 & 0.0 & 2.8 & 1.2 & 4.0 & 0.0 & 0.0 \\
\hline Net domestic financing & 23.2 & 69.7 & -2.0 & -40.3 & -1.5 & -42.3 & -3.5 & -10.9 & 22.5 \\
\hline ECCB (net) & -5.8 & 12.6 & -0.4 & 0.4 & 2.6 & 0.0 & 2.3 & 0.0 & 0.0 \\
\hline Commercial banks (net) 5/ & 26.5 & 54.4 & 5.5 & -36.8 & 3.7 & -31.3 & 9.2 & -11.7 & 17.1 \\
\hline Domestic debt & 2.5 & 2.7 & -7.2 & -3.9 & -7.8 & -11.0 & -14.9 & 0.8 & 5.4 \\
\hline Of which: Banking system & 1.5 & 0.0 & -0.4 & 1.3 & 3.3 & 0.9 & 2.9 & 0.8 & 4.7 \\
\hline Divestment/privatization proceeds & 8.8 & 36.0 & 47.7 & 2.7 & 2.6 & 50.4 & 50.3 & 44.6 & 2.4 \\
\hline Expenditure arrears $6 /$ & 31.2 & -14.6 & 7.1 & 13.0 & -20.8 & 20.1 & -13.7 & -2.4 & 4.6 \\
\hline Of which: Excluding arrears from debt exchange & 26.6 & -19.2 & 4.8 & 10.7 & -23.1 & 15.5 & -18.3 & -7.0 & 0.0 \\
\hline \multicolumn{10}{|l|}{ Memorandum items: } \\
\hline Nominal GDP (market prices) & 1,524 & 1,641 & 1,724 & 1,724 & 1,724 & 1,724 & 1,724 & 1,806 & 1,806 \\
\hline Stock of expenditure arrears $6 /$ & 51.8 & 37.2 & 44.3 & 27.8 & 23.5 & 57.3 & 23.5 & 54.9 & 28.1 \\
\hline Of which: Excluding arrears from debt exchange & 47.2 & 28.0 & 32.8 & 14.0 & 9.7 & 43.5 & 9.7 & 36.5 & 9.7 \\
\hline
\end{tabular}

Sources: Ministry of Finance; and Fund staff estimates and projections.

$1 /$ Corresponds to the staff supplement in IMF Country Report No. 09/95 with all ratios expressed relative to the revised GDP.

2/ VAT will be introduced on February 1, 2010.

$3 /$ Includes the transfer to the budget of PetroCaribe financing beyond that needed to repay related debt.

4/ The 2008 budget figures are adjusted to include interest arrears to nonparticipating creditors in the debt exchange.

5/ Excludes commercial bank holdings of government paper, which are shown in the following two lines.

6/ Includes EC \$4.6 million arrears each year from 2005 onwards on interest to nonparticipating creditors in the debt exchange. 
Table 2a. Grenada: Central Government Finances, 2006-09

(In percent of GDP, unless noted otherwise)

\begin{tabular}{|c|c|c|c|c|c|c|c|c|c|}
\hline & \multirow{3}{*}{$\begin{array}{r}\text { Actual } \\
2006\end{array}$} & \multirow{3}{*}{$\begin{array}{r}\text { Actual } \\
2007\end{array}$} & \multicolumn{5}{|c|}{2008} & \multirow[b]{2}{*}{ Prog. 1/ } & \multirow[b]{2}{*}{ Proj. } \\
\hline & & & Prel. & Prog. 1/ & Prel. & Prog. 1/ & Prel. & & \\
\hline & & & First Half & \multicolumn{2}{|c|}{ Second Half } & \multicolumn{2}{|c|}{ Full Year } & \multicolumn{2}{|c|}{2009} \\
\hline Total revenue and grants & 33.6 & 27.2 & 15.1 & 15.7 & 16.2 & 30.8 & 31.3 & 30.7 & 26.7 \\
\hline Total revenue & 24.9 & 26.1 & 13.5 & 12.8 & 13.4 & 26.3 & 26.9 & 27.0 & 24.2 \\
\hline Current revenue & 24.9 & 26.1 & 13.5 & 12.8 & 13.4 & 26.3 & 26.9 & 27.0 & 24.2 \\
\hline Tax revenue $2 /$ & 23.2 & 24.5 & 12.3 & 11.8 & 12.8 & 24.1 & 25.2 & 25.2 & 22.5 \\
\hline Taxes on income and profits & 3.7 & 4.6 & 2.3 & 2.4 & 3.1 & 4.7 & 5.5 & 4.5 & 4.5 \\
\hline Taxes on property & 1.5 & 1.8 & 1.0 & 0.5 & 0.5 & 1.5 & 1.5 & 1.5 & 1.7 \\
\hline Taxes on domestic goods and services & 4.5 & 4.3 & 2.5 & 1.9 & 2.1 & 4.4 & 4.6 & 4.8 & 4.6 \\
\hline Taxes on international transactions & 13.5 & 13.9 & 6.5 & 7.0 & 7.1 & 13.5 & 13.6 & 14.4 & 11.7 \\
\hline Nontax revenue & 1.7 & 1.6 & 1.2 & 1.0 & 0.6 & 2.1 & 1.8 & 1.8 & 1.7 \\
\hline Capital revenue & 0.0 & 0.0 & 0.0 & 0.0 & 0.0 & 0.0 & 0.0 & 0.0 & 0.0 \\
\hline Grants 3/ & 8.7 & 1.1 & 1.6 & 3.0 & 2.7 & 4.5 & 4.3 & 3.6 & 2.5 \\
\hline Total expenditure & 40.0 & 35.3 & 19.8 & 15.3 & 17.2 & 35.1 & 37.0 & 33.0 & 31.6 \\
\hline Current expenditure & 21.2 & 21.7 & 12.0 & 12.4 & 12.5 & 24.4 & 24.5 & 23.0 & 22.7 \\
\hline Current primary expenditure & 19.1 & 19.4 & 10.8 & 10.9 & 11.1 & 21.7 & 22.0 & 19.9 & 19.7 \\
\hline Salaries and allowances & 10.0 & 9.7 & 5.6 & 5.9 & 6.0 & 11.6 & 11.6 & 10.7 & 10.5 \\
\hline Wages and salaries & 8.9 & 8.6 & 5.0 & 5.3 & 5.4 & 10.3 & 10.4 & 9.2 & 9.0 \\
\hline Personnel allowances & 1.1 & 1.1 & 0.6 & 0.7 & 0.6 & 1.3 & 1.2 & 1.5 & 1.5 \\
\hline Goods and services & 4.7 & 4.8 & 2.2 & 2.2 & 2.7 & 4.4 & 4.9 & 4.3 & 4.1 \\
\hline Interest 4/ & 2.1 & 2.3 & 1.2 & 1.5 & 1.4 & 2.7 & 2.6 & 3.1 & 3.0 \\
\hline Domestic & 0.7 & 0.8 & 0.3 & 0.8 & 0.7 & 1.1 & 1.0 & 1.1 & 1.0 \\
\hline Foreign & 1.4 & 1.5 & 0.9 & 0.8 & 0.7 & 1.6 & 1.6 & 2.0 & 2.0 \\
\hline Transfers and subsidies & 4.5 & 4.9 & 3.0 & 2.8 & 2.4 & 5.8 & 5.4 & 4.9 & 5.1 \\
\hline Capital expenditure & 18.8 & 13.6 & 7.8 & 2.9 & 4.7 & 10.7 & 12.5 & 10.0 & 8.9 \\
\hline Current balance & 3.7 & 4.4 & 1.5 & 0.3 & 0.9 & 1.8 & 2.4 & 4.0 & 1.5 \\
\hline Primary balance (excluding grants) & -13.0 & -6.9 & -5.1 & -1.0 & -2.4 & -6.1 & -7.5 & -2.9 & -4.4 \\
\hline Primary balance (including grants) & -4.3 & -5.8 & -3.5 & 1.9 & 0.4 & -1.6 & -3.2 & 0.8 & -1.9 \\
\hline Overall balance (excluding grants) & -15.0 & -9.2 & -6.3 & -2.5 & -3.8 & -8.8 & -10.0 & -6.0 & -7.4 \\
\hline Overall balance (including grants) & -6.4 & -8.2 & -4.7 & 0.4 & -1.0 & -4.3 & -5.7 & -2.4 & -4.9 \\
\hline Statistical discrepancy & -1.4 & 0.2 & 1.4 & -1.4 & -0.2 & 0.0 & 1.3 & 0.0 & 0.0 \\
\hline Financing & 8.2 & 8.0 & 3.3 & 1.0 & 1.2 & 4.3 & 4.5 & 2.4 & 4.9 \\
\hline Net external financing & 3.6 & 2.4 & 0.2 & 2.4 & 2.4 & 2.6 & 2.6 & 0.6 & 3.2 \\
\hline Net amortization & 3.6 & 2.5 & 0.1 & 2.4 & 2.2 & 2.6 & 2.3 & 0.6 & 3.2 \\
\hline Disbursements & 4.8 & 3.8 & 1.1 & 3.1 & 2.8 & 4.1 & 3.8 & 2.1 & 4.7 \\
\hline Amortization & -1.2 & -1.4 & -0.9 & -0.6 & -0.6 & -1.5 & -1.5 & -1.5 & -1.5 \\
\hline Change in government assets & 0.0 & 0.0 & 0.1 & 0.0 & 0.2 & 0.1 & 0.2 & 0.0 & 0.0 \\
\hline Net domestic financing & 1.5 & 4.2 & -0.1 & -2.3 & -0.1 & -2.5 & -0.2 & -0.6 & 1.2 \\
\hline ECCB (net) & -0.4 & 0.8 & 0.0 & 0.0 & 0.2 & 0.0 & 0.1 & 0.0 & 0.0 \\
\hline Commercial banks (net) $5 /$ & 1.7 & 3.3 & 0.3 & -2.1 & 0.2 & -1.8 & 0.5 & -0.6 & 0.9 \\
\hline Domestic debt & 0.2 & 0.2 & -0.4 & -0.2 & -0.5 & -0.6 & -0.9 & 0.0 & 0.3 \\
\hline Of which: Banking system & 0.1 & 0.0 & 0.0 & 0.1 & 0.2 & 0.0 & 0.2 & 0.0 & 0.3 \\
\hline Divestment/privatization proceeds & 0.6 & 2.2 & 2.8 & 0.2 & 0.2 & 2.9 & 2.9 & 2.5 & 0.1 \\
\hline Expenditure arrears $6 /$ & 2.0 & -0.9 & 0.4 & 0.8 & -1.2 & 1.2 & -0.8 & -0.1 & 0.3 \\
\hline Of which: Excluding arrears from debt exchange & 1.7 & -1.2 & 0.3 & 0.6 & -1.3 & 0.9 & -1.1 & -0.4 & 0.0 \\
\hline \multicolumn{10}{|l|}{ Memorandum items: } \\
\hline Nominal GDP (market prices, EC\$ millions) & 1,524 & 1,641 & 1,724 & 1,724 & 1,724 & 1,724 & 1,724 & 1,806 & 1,806 \\
\hline Stock of expenditure arrears $6 /$ & 3.4 & 2.3 & 2.6 & 3.3 & 1.4 & 3.3 & 1.4 & 3.0 & 1.6 \\
\hline Of which: Excluding arrears from debt exchange & 3.1 & 1.7 & 1.9 & 2.5 & 0.6 & 2.5 & 0.6 & 2.0 & 0.5 \\
\hline
\end{tabular}

Sources: Ministry of Finance; and Fund staff estimates and projections.

$1 /$ Corresponds to the staff supplement in IMF Country Report No. 09/95 with all ratios expressed relative to the revised GDP

2/ VAT will be introduced on February 1, 2010.

$3 /$ Includes the transfer to the budget of PetroCaribe financing beyond that needed to repay related debt.

4/ The 2008 budget figures are adjusted to include interest arrears to nonparticipating creditors in the debt exchange.

5/ Excludes commercial bank holdings of government paper, which are shown in the following two lines.

$6 /$ Includes EC \$4.6 million arrears each year from 2005 onwards on interest to nonparticipating creditors in the debt exchange. 
Table 3. Grenada: Summary Accounts of the Banking System, 2006-10

\begin{tabular}{|c|c|c|c|c|c|c|}
\hline & \multirow[b]{2}{*}{2006} & \multirow[b]{2}{*}{2007} & \multirow{2}{*}{ June } & \multirow{2}{*}{ Dec. } & \multicolumn{2}{|c|}{ Proj. } \\
\hline & & & & & 2009 & 2010 \\
\hline & \multicolumn{6}{|c|}{ (In millions of Eastern Caribbean dollars, end of period) } \\
\hline Net foreign assets & 421.5 & 405.6 & 344.6 & 248.8 & 187.5 & 130.2 \\
\hline ECCB (imputed reserves) & 269.4 & 298.2 & 306.3 & 281.0 & 253.2 & 241.4 \\
\hline Commercial banks & 152.0 & 107.4 & 38.3 & -32.2 & -65.7 & -111.2 \\
\hline Net domestic assets & $1,060.9$ & $1,239.1$ & $1,349.1$ & $1,456.9$ & $1,534.9$ & $1,629.2$ \\
\hline Public sector credit (net) & -36.0 & -12.5 & -2.5 & 4.4 & 36.3 & 36.8 \\
\hline Central government & 37.3 & 104.3 & 109.1 & 118.7 & 140.6 & 141.1 \\
\hline $\mathrm{ECCB}$ & -7.5 & 5.0 & 4.7 & 6.4 & 6.4 & 6.4 \\
\hline Commercial banks & 44.9 & 99.3 & 104.4 & 112.3 & 134.2 & 134.7 \\
\hline Net credit to rest of public sector & -73.3 & -116.8 & -111.6 & -114.3 & -104.3 & -104.3 \\
\hline National Insurance Scheme & -86.6 & -112.5 & -119.5 & -135.1 & -130.1 & -130.1 \\
\hline Other & 13.2 & -4.3 & 7.9 & 20.7 & 25.7 & 25.7 \\
\hline Credit to private sector & $1,223.9$ & $1,409.2$ & $1,440.1$ & $1,562.8$ & $1,608.9$ & $1,702.7$ \\
\hline Net credit to nonbank financial institutions & -23.3 & -27.2 & 3.2 & -14.0 & -14.1 & -14.0 \\
\hline Other items (net) & -103.7 & -130.4 & -91.6 & -96.2 & -96.2 & -96.2 \\
\hline Broad money & $1,482.4$ & $1,644.7$ & $1,693.8$ & $1,705.7$ & $1,722.4$ & $1,759.4$ \\
\hline Money & 309.5 & 355.8 & 358.0 & 338.6 & 336.8 & 335.4 \\
\hline Currency in circulation & 104.5 & 107.8 & 106.0 & 103.4 & 102.8 & 102.4 \\
\hline Demand deposits & 205.0 & 248.0 & 252.0 & 235.2 & 234.0 & 233.0 \\
\hline Quasi-money & $1,172.9$ & $1,288.9$ & $1,335.8$ & $1,367.1$ & $1,385.6$ & $1,424.0$ \\
\hline Time deposits & 201.2 & 254.1 & 281.6 & 281.3 & 283.1 & 295.7 \\
\hline Savings deposits & 894.9 & 914.2 & 967.3 & 977.3 & $1,002.7$ & $1,025.0$ \\
\hline \multirow[t]{2}{*}{ Foreign currency deposits } & 76.9 & 120.7 & 86.9 & 108.6 & 99.7 & 103.3 \\
\hline & \multicolumn{6}{|c|}{ (Annual percentage change) } \\
\hline Net foreign assets & -20.7 & -3.8 & -19.4 & -38.7 & -24.6 & -30.6 \\
\hline Net domestic assets & 13.2 & 16.8 & 19.0 & 17.6 & 5.3 & 6.1 \\
\hline Credit to private sector & 12.5 & 15.1 & 14.8 & 10.9 & 3.0 & 5.8 \\
\hline Broad money & 0.9 & 11.0 & 8.5 & 3.7 & 1.0 & 2.2 \\
\hline Money & -1.9 & 15.0 & 7.2 & -4.8 & -0.5 & -0.4 \\
\hline \multirow[t]{2}{*}{ Quasi-money 1/ } & 1.7 & 9.9 & 8.8 & 6.1 & 1.3 & 2.8 \\
\hline & \multicolumn{6}{|c|}{ (Percent contribution compared to $\mathrm{M} 2$ at the beginning of the year) } \\
\hline Net foreign assets & -7.5 & -1.1 & -3.7 & -9.5 & -3.6 & -3.3 \\
\hline Net domestic assets & 8.4 & 12.0 & 6.7 & 13.2 & 4.6 & 5.5 \\
\hline Public sector credit (net) & -0.3 & 1.6 & 0.6 & 1.0 & 1.9 & 0.0 \\
\hline Of which: Central government & 1.5 & 4.5 & 0.3 & 0.9 & 1.3 & 0.0 \\
\hline Credit to private sector & 9.2 & 12.5 & 1.9 & 9.3 & 2.7 & 5.4 \\
\hline Net credit to nonbank financial inst. & 0.4 & -0.3 & 1.9 & 0.8 & 0.0 & 0.0 \\
\hline Other items (net) & -0.9 & -1.8 & 2.4 & 2.1 & 0.0 & 0.0 \\
\hline \multicolumn{7}{|l|}{ Memorandum item: } \\
\hline Income velocity $2 /$ & 1.2 & 1.2 & 1.2 & 1.1 & 1.1 & 1.1 \\
\hline
\end{tabular}

Sources: ECCB; Ministry of Finance and Planning; and Fund staff estimates.

$1 /$ Including resident foreign currency deposits.

2/ Nominal GDP at market prices divided by liabilities to the private sector. 
Table 4. Grenada: Summary Balance of Payments, 2006-14

\begin{tabular}{|c|c|c|c|c|c|c|c|c|c|}
\hline & \multirow[b]{2}{*}{$20061 /$} & \multirow[b]{2}{*}{$20071 /$} & \multirow{2}{*}{ Est. } & \multicolumn{6}{|c|}{ Projections } \\
\hline & & & & 2009 & 2010 & 2011 & 2012 & 2013 & 2014 \\
\hline & \multicolumn{9}{|c|}{ (In millions of U.S. dollars) } \\
\hline Current account & -188.4 & -254.6 & -269.9 & -214.1 & -210.7 & -219.9 & -228.8 & -229.7 & -239.6 \\
\hline Trade balance & -264.1 & -287.2 & -306.5 & -231.4 & -239.5 & -257.0 & -272.2 & -288.8 & -315.4 \\
\hline Exports (f.o.b.) 2/ & 32.3 & 40.7 & 33.0 & 32.7 & 35.8 & 40.5 & 45.4 & 50.8 & 56.7 \\
\hline Imports (f.o.b.) & -296.4 & -327.9 & -339.5 & -264.1 & -275.3 & -297.5 & -317.6 & -339.6 & -372.1 \\
\hline Of which: Mineral fuels & -43.6 & -53.6 & -73.3 & -33.0 & -39.4 & -44.8 & -49.6 & -53.4 & -55.5 \\
\hline Travel (net) & 88.2 & 116.7 & 112.0 & 96.1 & 104.2 & 115.7 & 130.5 & 150.7 & 177.0 \\
\hline Other services (net) & -59.4 & -80.2 & -81.5 & -74.8 & -73.4 & -73.9 & -78.6 & -83.5 & -90.4 \\
\hline Income (net) & -31.4 & -34.7 & -37.5 & -34.9 & -35.9 & -38.7 & -42.8 & -42.9 & -46.1 \\
\hline Transfers (net) & 78.3 & 30.7 & 43.6 & 30.9 & 33.9 & 34.0 & 34.3 & 34.9 & 35.4 \\
\hline Capital and financial account & 198.4 & 248.4 & 273.8 & 182.7 & 203.7 & 221.5 & 232.2 & 233.0 & 245.8 \\
\hline Capital account (transfers) & 23.5 & 24.1 & 19.8 & 19.8 & 20.1 & 20.7 & 21.6 & 22.7 & 22.0 \\
\hline Financial account & 175.0 & 224.2 & 254.0 & 162.9 & 183.6 & 200.7 & 210.6 & 210.3 & 223.8 \\
\hline Public sector borrowing & 26.1 & 27.4 & 35.2 & 30.2 & 33.9 & 23.2 & 24.2 & 15.5 & 16.0 \\
\hline Public sector amortization & -9.1 & -9.7 & -10.3 & -11.6 & -13.8 & -11.1 & -13.9 & -17.1 & -13.4 \\
\hline Direct investment (net) & 89.8 & 174.2 & 161.2 & 102.3 & 115.8 & 137.2 & 148.8 & 157.2 & 166.5 \\
\hline Portfolio investment (net) & -0.7 & -1.0 & 9.3 & 9.8 & 10.0 & 10.4 & 10.9 & 11.4 & 11.9 \\
\hline Change in NFA of commercial banks & 46.3 & 16.5 & 51.7 & 12.4 & 16.9 & 19.7 & 18.9 & 20.9 & 22.2 \\
\hline Other investments (net) & 22.6 & 16.8 & 6.9 & 19.8 & 20.9 & 21.2 & 21.7 & 22.4 & 20.6 \\
\hline Net errors and omissions & -5.1 & 19.3 & -15.9 & 0.0 & 0.0 & 0.0 & 0.0 & 0.0 & 0.0 \\
\hline Overall balance & 5.0 & 13.0 & -12.0 & -31.5 & -6.9 & 1.5 & 3.4 & 3.3 & 6.3 \\
\hline Available financing & -5.0 & -13.0 & 12.0 & 19.8 & 6.9 & -1.5 & -3.4 & -3.3 & -6.3 \\
\hline Change in imputed reserves & -5.6 & -10.7 & 6.4 & 10.3 & 4.4 & -1.3 & -2.9 & -2.9 & -3.3 \\
\hline IMF purchases and disbursements & 2.3 & 0.0 & 8.5 & 11.8 & 2.6 & 0.0 & 0.0 & 0.0 & 0.0 \\
\hline IMF repurchases and repayments & -1.6 & -2.2 & -2.9 & -2.2 & 0.0 & -0.2 & -0.5 & -0.5 & -3.0 \\
\hline Other & -0.1 & -0.1 & 0.0 & 0.0 & 0.0 & 0.0 & 0.0 & 0.0 & 0.0 \\
\hline \multirow[t]{2}{*}{ Financing gap $3 /$} & \multicolumn{9}{|c|}{11.6} \\
\hline & \multicolumn{9}{|c|}{ (In percent of GDP) } \\
\hline Current account & -33.4 & -41.9 & -42.3 & -32.0 & -30.3 & -29.9 & -29.4 & -27.8 & -27.4 \\
\hline Trade balance & -46.8 & -47.2 & -48.0 & -34.6 & -34.5 & -35.0 & -35.0 & -35.0 & -36.0 \\
\hline Exports of goods & 5.7 & 6.7 & 5.2 & 4.9 & 5.2 & 5.5 & 5.8 & 6.2 & 6.5 \\
\hline Imports of goods & -52.5 & -53.9 & -53.2 & -39.5 & -39.6 & -40.5 & -40.8 & -41.2 & -42.5 \\
\hline Travel (net) & 15.6 & 19.2 & 17.5 & 14.4 & 15.0 & 15.8 & 16.8 & 18.3 & 20.2 \\
\hline Other services (net) & -10.5 & -13.2 & -12.8 & -11.2 & -10.6 & -10.1 & -10.1 & -10.1 & -10.3 \\
\hline Income and current transfers (net) & 8.3 & -0.7 & 1.0 & -0.6 & -0.3 & -0.6 & -1.1 & -1.0 & -1.2 \\
\hline Capital and financial account & 35.2 & 40.9 & 42.9 & 27.3 & 29.3 & 30.1 & 29.8 & 28.2 & 28.1 \\
\hline Public sector & 3.0 & 2.9 & 3.9 & 2.8 & 2.9 & 1.6 & 1.3 & -0.2 & 0.3 \\
\hline Private sector & 32.1 & 37.9 & 39.0 & 24.5 & 26.4 & 28.5 & 28.5 & 28.4 & 27.8 \\
\hline Of which: Direct investment (net) & 15.9 & 28.7 & 25.2 & 15.3 & 16.7 & 18.7 & 19.1 & 19.0 & 19.0 \\
\hline Overall balance & 0.9 & 2.1 & -1.9 & -4.7 & -1.0 & 0.2 & 0.4 & 0.4 & 0.7 \\
\hline \multirow[t]{2}{*}{ External public and publicly guaranteed debt } & 81.0 & 78.7 & 79.2 & 79.9 & 80.2 & 77.4 & 74.3 & 69.9 & 65.8 \\
\hline & \multicolumn{9}{|c|}{ (Annual percentage change) } \\
\hline Exports of goods & -1.7 & 26.0 & -19.0 & -0.8 & 9.5 & 13.0 & 12.1 & 12.1 & 11.5 \\
\hline Imports of goods & -0.8 & 10.6 & 3.5 & -22.2 & 4.2 & 8.1 & 6.7 & 6.9 & 9.6 \\
\hline Travel (net) & 27.4 & 32.3 & -4.0 & -14.3 & 8.5 & 11.0 & 12.8 & 15.5 & 17.4 \\
\hline Memorandum items: & \multicolumn{9}{|c|}{ (In millions of U.S. dollars) } \\
\hline External public and publicly guaranteed debt & 457.2 & 478.7 & 506.0 & 534.1 & 556.8 & 568.6 & 578.4 & 576.4 & 576.0 \\
\hline
\end{tabular}

Sources: Eastern Caribbean Central Bank (ECCB); Ministry of Finance; and Fund staff estimates and projections.

$1 /$ Incorporates revised historical data from the ECCB.

2/ Re-exports increased sharply in 2007 upon completion of construction related to the 2007 Cricket World Cup.

$3 /$ Includes proposed augmentation (US $\$ 6.5$ million) and possible reschedulings from the Paris Club and Taiwan POC (US\$5.1 million). 
Table 5. Grenada: Public Sector Debt, 2008

(Year end, in millions of U.S. dollars)

\begin{tabular}{|c|c|c|c|}
\hline & \multirow[b]{2}{*}{ Stock } & \multicolumn{2}{|c|}{ Percent of } \\
\hline & & Total Debt & GDP \\
\hline Public sector debt $1 /$ & 693.3 & 100.0 & 108.6 \\
\hline Central government debt & 600.3 & 86.6 & 94.0 \\
\hline Central-government guaranteed debt & 54.8 & 7.9 & 8.6 \\
\hline Other public sector debt & 38.2 & 5.5 & 6.0 \\
\hline External debt & 506.0 & 73.0 & 79.2 \\
\hline Central government & 445.8 & 64.3 & 69.8 \\
\hline Multilateral & 167.1 & 24.1 & 26.2 \\
\hline CDB & 100.2 & 14.4 & 15.7 \\
\hline IDA & 36.0 & 5.2 & 5.6 \\
\hline IBRD & 11.5 & 1.7 & 1.8 \\
\hline IMF & 13.0 & 1.9 & 2.0 \\
\hline Other multilateral & 6.5 & 0.9 & 1.0 \\
\hline Official bilateral & 79.3 & 11.4 & 12.4 \\
\hline Paris Club & 17.9 & 2.6 & 2.8 \\
\hline Belgium & 7.3 & 1.0 & 1.1 \\
\hline France & 4.3 & 0.6 & 0.7 \\
\hline Russian Federation & 0.2 & 0.0 & 0.0 \\
\hline United Kingdom & 3.3 & 0.5 & 0.5 \\
\hline United States & 2.9 & 0.4 & 0.5 \\
\hline Non-Paris Club & 61.4 & 8.9 & 9.6 \\
\hline Kuwait & 18.5 & 2.7 & 2.9 \\
\hline Taiwan Province of China & 20.3 & 2.9 & 3.2 \\
\hline Trinidad and Tobago & 17.1 & 2.5 & 2.7 \\
\hline Other bilateral & 5.6 & 0.8 & 0.9 \\
\hline Commercial, total & 199.4 & 28.8 & 31.2 \\
\hline Restructured bonds & 193.5 & 27.9 & 30.3 \\
\hline Unrestructured bonds & 5.8 & 0.8 & 0.9 \\
\hline $\begin{array}{l}\text { Central government guaranteed } \\
\text { Of which }\end{array}$ & 22.0 & 3.2 & 3.4 \\
\hline Paris Club & 6.0 & 0.9 & 0.9 \\
\hline Other public sector $2 /$ & 38.2 & 5.5 & 6.0 \\
\hline Domestic debt & 187.3 & 27.0 & 29.3 \\
\hline Central government & 154.5 & 22.3 & 24.2 \\
\hline Restructured Bonds & 68.1 & 9.8 & 10.7 \\
\hline Unrestructured bonds & 2.8 & 0.4 & 0.4 \\
\hline Treasury bills & 29.1 & 4.2 & 4.6 \\
\hline Commercial bank loans & 21.8 & 3.1 & 3.4 \\
\hline Overdraft & 13.7 & 2.0 & 2.1 \\
\hline Domestic arrears & 3.6 & 0.5 & 0.6 \\
\hline Compensation claims & 14.8 & 2.1 & 2.3 \\
\hline Other & 0.6 & 0.1 & 0.1 \\
\hline Central-government guaranteed & 32.8 & 4.7 & 5.1 \\
\hline $\begin{array}{l}\text { Memorandum item: } \\
\text { Nominal GDP }\end{array}$ & 638.5 & & \\
\hline
\end{tabular}

Sources: Grenada authorities; and Fund staff estimates.

$1 /$ Includes central government liabilities to the National Insurance Scheme.

2/ Includes PetroCaribe-related borrowing. 
Table 6. Grenada: Reviews and Disbursements Under the PRGF Arrangement, 2006-10

\begin{tabular}{|c|c|c|c|c|}
\hline Date & Conditions & Disbursement & Percent of Quota & Availability date \\
\hline April 2006 & Board approval of PRGF arrangement & SDR $1,560,000$ & 13.3 & April 15, 2006 \\
\hline July 2008 & $\begin{array}{l}\text { Observance of end-June } 2006 \text { performance criteria, completion of first review } \\
\text { and adopt conditions for second year of the arrangement }\end{array}$ & SDR $2,980,000$ & 25.5 & July 15, 2008 \\
\hline December 2008 & $\begin{array}{l}\text { Observance of end-June } 2008 \text { performance criteria and completion of } \\
\text { second review }\end{array}$ & SDR $2,410,000$ & 20.6 & October 15, 2008 \\
\hline June 2009 & $\begin{array}{l}\text { Observance of end-December } 2008 \text { performance criteria, completion of third review, } \\
\text { and adopt conditions for third year of the arrangement }\end{array}$ & SDR $3,875,0001 /$ & 33.1 & April 15, 2009 \\
\hline October 2009 & Observance of end-June 2009 performance criteria and completion of fourth review & SDR $3,875,0001 /$ & 33.1 & October 15, 2009 \\
\hline April 2010 & Observance of end-November 2009 performance criteria and completion of fifth review & SDR $1,680,000$ & 14.4 & February 28, 2010 \\
\hline Total & & SDR $16,380,000$ & 140.0 & \\
\hline
\end{tabular}

Source: Fund staff.

1/ Includes proposed augmentation of 37.5 percent of quota (SDR 4,390,000) phased equally between these two disbursements. 
Table 7. Grenada: Indicators of Capacity to Repay the Fund, 2007-16 1/

(In millions of SDRs, unless otherwise indicated)

\begin{tabular}{|c|c|c|c|c|c|c|c|c|c|c|}
\hline & \multirow[b]{2}{*}{2007} & \multirow[b]{2}{*}{2008} & \multicolumn{6}{|c|}{ Projections } & \multirow[b]{2}{*}{2015} & \multirow[b]{2}{*}{2016} \\
\hline & & & 2009 & 2010 & 2011 & 2012 & 2013 & 2014 & & \\
\hline Fund obligations based on existing credit & 1.7 & 1.9 & 1.2 & 0.0 & 0.2 & 0.4 & 0.3 & 1.4 & 1.4 & 1.3 \\
\hline Repurchases and repayments & 1.5 & 1.8 & 1.1 & 0.0 & 0.2 & 0.3 & 0.3 & 1.4 & 1.4 & 1.2 \\
\hline Charges and interest & 0.3 & 0.1 & 0.1 & 0.0 & 0.0 & 0.0 & 0.0 & 0.0 & 0.0 & 0.0 \\
\hline Fund obligations based on existing and prospective credit & 1.7 & 1.9 & 1.2 & 0.1 & 0.2 & 0.4 & 0.4 & 2.0 & 3.3 & 3.2 \\
\hline Repurchases and repayments & 1.5 & 1.8 & 1.1 & 0.0 & 0.2 & 0.3 & 0.3 & 2.0 & 3.3 & 3.1 \\
\hline Charges and interest & 0.3 & 0.1 & 0.1 & 0.1 & 0.1 & 0.1 & 0.1 & 0.1 & 0.1 & 0.1 \\
\hline \multicolumn{11}{|l|}{ Fund obligations based on existing and prospective credit } \\
\hline In millions of U.S. dollars & 2.7 & 3.1 & 1.8 & 0.1 & 0.4 & 0.6 & 0.6 & 3.2 & 5.2 & 4.9 \\
\hline In percent of exports of goods and services & 1.4 & 1.7 & 1.1 & 0.1 & 0.2 & 0.3 & 0.3 & 1.2 & 1.9 & 1.7 \\
\hline In percent of debt service 2 / & 10.5 & 10.1 & 5.0 & 0.5 & 1.3 & 1.7 & 1.2 & 5.6 & 8.2 & 6.6 \\
\hline In percent of GDP & 0.4 & 0.5 & 0.3 & 0.0 & 0.1 & 0.1 & 0.1 & 0.4 & 0.6 & 0.5 \\
\hline In percent of Imputed Net International Reserves & 2.4 & 3.0 & 1.9 & 0.1 & 0.4 & 0.6 & 0.6 & 3.2 & 5.1 & 4.8 \\
\hline In percent of quota & 14.8 & 16.5 & 10.0 & 0.7 & 2.1 & 3.3 & 3.3 & 17.4 & 28.5 & 27.1 \\
\hline \multicolumn{11}{|l|}{ Outstanding Fund credit (end of period) } \\
\hline In millions of SDRs & 4.9 & 8.4 & 15.1 & 16.7 & 16.6 & 16.3 & 16.0 & 14.0 & 10.7 & 7.6 \\
\hline In millions of U.S. dollars & 7.7 & 13.1 & 23.5 & 26.1 & 25.8 & 25.3 & 24.9 & 21.8 & 16.7 & 11.9 \\
\hline In percent of exports of goods and services & 4.1 & 7.4 & 14.6 & 15.0 & 13.5 & 11.9 & 10.3 & 8.0 & 6.1 & 4.0 \\
\hline In percent of debt service $2 /$ & 30.4 & 43.1 & 64.7 & 100.8 & 88.7 & 72.3 & 50.0 & 38.4 & 26.2 & 15.9 \\
\hline In percent of GDP & 1.3 & 2.1 & 3.5 & 3.8 & 3.5 & 3.3 & 3.0 & 2.5 & 1.8 & 1.2 \\
\hline In percent of Imputed Net International Reserves & 6.9 & 12.6 & 25.0 & 29.2 & 28.5 & 27.1 & 25.8 & 22.0 & 16.5 & 11.5 \\
\hline In percent of quota & 41.5 & 71.9 & 128.8 & 143.1 & 141.8 & 139.1 & 136.5 & 119.8 & 91.8 & 65.1 \\
\hline Net use of Fund credit & -1.5 & 3.6 & 6.7 & 1.7 & -0.2 & -0.3 & -0.3 & -2.0 & -3.3 & -3.1 \\
\hline Disbursements & 0.0 & 5.4 & 7.8 & 1.7 & 0.0 & 0.0 & 0.0 & 0.0 & 0.0 & 0.0 \\
\hline Repayments and Repurchases & 1.5 & 1.8 & 1.1 & 0.0 & 0.2 & 0.3 & 0.3 & 2.0 & 3.3 & 3.1 \\
\hline \multicolumn{11}{|l|}{ Memorandum items: } \\
\hline Exports of goods and services (in millions of US\$) & 188.1 & 176.8 & 160.2 & 173.8 & 191.6 & 213.1 & 240.8 & 273.7 & 275.9 & 293.7 \\
\hline Debt service (in millions of US\$) 2/ & 25.2 & 30.4 & 36.3 & 25.9 & 29.2 & 35.1 & 49.8 & 57.5 & 65.6 & 76.3 \\
\hline GDP (in millions of US\$) & 607.9 & 638.5 & 668.8 & 694.4 & 734.6 & 778.6 & 825.1 & 875.3 & 928.5 & 985.0 \\
\hline Imputed Net International Reserves (in millions of US\$) & 110.4 & 104.1 & 93.8 & 89.4 & 90.7 & 93.6 & 96.5 & 99.3 & 101.1 & 102.9 \\
\hline Quota & 11.7 & 11.7 & 11.7 & 11.7 & 11.7 & 11.7 & 11.7 & 11.7 & 11.7 & 11.7 \\
\hline
\end{tabular}

Source: Fund staff estimates and projections.

$1 /$ Incorporates proposed augmentation of 37.5 percent of quota.

2/ Total debt service including the Fund. 
Table 8. Grenada: Vulnerability Indicators, 2004-08

\begin{tabular}{|c|c|c|c|c|c|}
\hline & 2004 & 2005 & 2006 & 2007 & $\begin{array}{r}\text { Est. } \\
2008 \\
\end{array}$ \\
\hline \multicolumn{6}{|l|}{ Real sector indicators } \\
\hline Real GDP growth (percent) & -5.7 & 11.0 & -2.3 & 4.5 & 0.3 \\
\hline $\mathrm{CPI}$ inflation (period average, in percent) & 2.3 & 3.5 & 4.2 & 3.9 & 8.0 \\
\hline \multicolumn{6}{|l|}{ Financial sector indicators } \\
\hline Total capital asset ratio of banks (locally incorporated) & 14.9 & 15.3 & 17.5 & 15.6 & 15.1 \\
\hline Of which: Tier 1 capital & 10.5 & 12.7 & 13.2 & 13.3 & 13.0 \\
\hline Liquid assets/total assets & 44.3 & 37.3 & 31.5 & 29.2 & 24.1 \\
\hline Liquid assets/current liabilities & 48.2 & 40.0 & 34.6 & 32.3 & 26.7 \\
\hline Total loans/total deposits & 57.6 & 66.0 & 73.2 & 75.9 & 80.0 \\
\hline Net liquid assets/total deposits & 44.2 & 35.4 & 28.6 & 26.4 & 19.8 \\
\hline Nonperforming loans/total loans & 6.0 & 5.5 & 3.7 & 3.5 & 3.5 \\
\hline Locally incorporated banks & 7.1 & 6.8 & 4.7 & 3.4 & 3.5 \\
\hline Foreign banks & 4.4 & 3.5 & 2.4 & 3.5 & 3.4 \\
\hline Provisions for loan losses /nonperforming assets & 81.9 & 79.4 & 67.4 & 57.7 & 44.9 \\
\hline Locally incorporated banks & 63.0 & 60.7 & 51.5 & 48.3 & 33.3 \\
\hline Foreign banks & 127.8 & 134.6 & 114.6 & 71.2 & 63.0 \\
\hline Gross government claims/total assets & 11.3 & 11.2 & 11.6 & 11.9 & 12.7 \\
\hline FX deposits/total deposits & 5.6 & 7.8 & 5.8 & 7.7 & 6.9 \\
\hline Net foreign currency exposure/capital (locally incorporated banks) & 221.4 & 138.4 & 101.4 & 65.7 & 47.1 \\
\hline Contingent liabilities/capital (locally incorporated banks) & 67.7 & 78.7 & 72.2 & 85.8 & 68.2 \\
\hline Ratio of bank's before-tax profits to average assets (percent) & 0.5 & 0.7 & 2.5 & 2.1 & 1.9 \\
\hline Broad money (percent change, 12-month basis) & 17.8 & -1.0 & 0.9 & 11.0 & 8.5 \\
\hline Private sector credit (percent change, 12-month basis) & 6.8 & 9.2 & 12.5 & 15.1 & 10.9 \\
\hline U.S. treasury bill rate (percent per annum) & 1.4 & 3.2 & 4.8 & 4.5 & 1.5 \\
\hline Treasury bill rate (percent per annum) $1 /$ & 6.0 & 6.0 & 6.5 & 6.5 & 6.3 \\
\hline \multicolumn{6}{|l|}{ External sector indicators } \\
\hline Exchange rate (per US\$, end of period) & 2.7 & 2.7 & 2.7 & 2.7 & 2.7 \\
\hline REER appreciation (percent change on 12-month basis, end of period) & -3.5 & 5.9 & -4.6 & 0.2 & 6.6 \\
\hline Exports of goods (percent change, 12-month basis) & -17.6 & -12.4 & -1.7 & 26.0 & -19.0 \\
\hline Imports of goods (percent change, 12-month basis) & -0.1 & 32.1 & -0.8 & 10.6 & 3.5 \\
\hline Travel receipts (gross, percent change, 12-month basis) & -18.7 & -5.4 & 30.8 & 27.7 & -2.9 \\
\hline Current account balance (percent of GDP) & -9.0 & -31.3 & -33.4 & -41.9 & -42.3 \\
\hline Capital and financial account balance (percent of GDP) & 24.0 & 35.3 & 35.2 & 40.9 & 42.9 \\
\hline Net FDI inflows (percent of GDP) & 13.9 & 12.7 & 15.9 & 28.7 & 25.2 \\
\hline Gross international reserves of the ECCB (in US\$ millions) & 632.4 & 600.8 & 696.0 & 764.5 & 759.0 \\
\hline Gross international reserves in months of current year imports in ECCU countries & 4.8 & 3.9 & 3.8 & 3.7 & 3.5 \\
\hline Gross international reserves to broad money in ECCU countries (percent) & 20.4 & 17.9 & 18.6 & 18.6 & 17.9 \\
\hline Public gross external debt (in US\$ million) & 415.6 & 437.0 & 457.2 & 478.7 & 506.0 \\
\hline Public gross external debt to exports of goods and services (percent) & 44.8 & 53.0 & 49.9 & 41.9 & 44.8 \\
\hline Public gross external interest payments to exports of goods and services (percent) & 13.7 & 6.5 & 7.5 & 7.2 & 8.9 \\
\hline Public gross external amortization payments to exports of goods and services (percent) & 8.6 & 6.0 & 4.9 & 8.4 & 7.4 \\
\hline Public gross external interest payments to fiscal revenue (percent) & 17.5 & 5.1 & 6.0 & 5.8 & 6.2 \\
\hline Public gross external amortization payments to fiscal revenue (percent) & 11.0 & 4.7 & 3.9 & 6.8 & 5.2 \\
\hline \multirow[t]{2}{*}{ Gross external financing requirement (in percent of GDP) } & 12.6 & 32.9 & 35.0 & 43.5 & 43.9 \\
\hline & \multicolumn{5}{|c|}{ (In percent of GDP) } \\
\hline \multicolumn{6}{|l|}{ Public sector indicators } \\
\hline Central government overall balance (after grants) & -2.6 & 0.4 & -6.4 & -8.2 & -5.7 \\
\hline Public and publicly-guaranteed gross external debt & 88.6 & 78.9 & 81.0 & 78.7 & 79.2 \\
\hline
\end{tabular}

Sources: Ministry of Finance; Eastern Caribbean Central Bank; and Fund staff estimates and projections.

1/ Rate on one-year treasury bills. 


\section{Annex: Summary of Appendices}

\section{Fund Relations}

Grenada's outstanding purchases as of end-April 2009 amounted to SDR 8.05 million (68.78 percent of quota). Grenada is a member of the ECCB, which manages monetary policy and the exchange system for its eight members. The common currency, the Eastern Caribbean dollar, has been pegged to the U.S. dollar at the rate of EC\$2.70 per U.S. dollar since July 1976. The last Article IV consultation was concluded by the Executive Board on September 26, 2007 (IMF Country Report No. 08/351). The next Article IV consultation is expected to take place in September 2009. CARTAC, MCM, FAD, and LEG have provided extensive technical assistance. A safeguards assessment was completed in 2007, and concluded that the ECCB continues to have appropriate control mechanisms in place.

\section{Relations with the World Bank Group ${ }^{20}$}

In September 2005, the Eastern Caribbean Sub-Region Country Assistance Strategy (CAS) for FY 2006-09 was presented to the Board of the World Bank. The strategy supports the sub-region's development agenda through two main pillars: (1) stimulating growth and improving competitiveness; and (2) reducing vulnerability, by promoting greater social inclusion and strengthening disaster risk management. There are nine active World Bank projects in Grenada for a net commitment of approximately US\$48.5 million.

\section{Relations with the Caribbean Development Bank ${ }^{21}$}

Grenada, since Hurricane Ivan in 2004, continues to benefit from special financing from the Caribbean Development Bank (CDB), designed to yield a concessionary grant element of 35 percent. These loans offer longer maturities and grace periods, as well as lower interest rates than those applied in the Bank's ordinary operations. Recently approved activities include the rockfall and landslip project, institutional strengthening, the school rehabilitation and reconstruction project, and project management training.

\section{Statistical Issues}

Grenada participates in the Fund's General Data Dissemination System (GDDS). Although data provision is sufficient for program monitoring, significant improvement is needed to facilitate effective surveillance by addressing weaknesses in coverage, timeliness and frequency of data.

\footnotetext{
${ }^{20}$ Adapted from text prepared by World Bank staff in April 2009.

${ }^{21}$ Adapted from text prepared by Caribbean Development Bank staff in April 2009.
} 


\title{
Attachment I: Letter of Intent
}

St. George's, Grenada

May 19, 2009

\author{
Mr. Dominique Strauss-Kahn \\ Managing Director \\ International Monetary Fund \\ Washington, D.C. 20431
}

Dear Mr. Strauss-Kahn:

We have made significant progress with our economic program since the second review of our arrangement under the PRGF in December 2008. Implementing the large fiscal adjustment in the second half of 2008 was challenging, but we are as a result better placed to deal with global turbulence. In light of the sharp slowdown in global growth and the continuing global financial crisis, we have decided to review our policy framework for 2009.

The attached Supplementary Memorandum of Economic Policies (SMEP) discusses progress in implementing our PRGF-supported program, and outlines policies, objectives, and macroeconomic framework for 2009 and beyond. It gives priority to mitigating the impact of the global economic slowdown, putting our debt on a sustainable trajectory, undertaking reforms to improve the business environment, and strengthening our capacity for economic and fiscal management.

Reflecting the strong measures taken after our government took office in July 2008, we have met all of our end-December 2008 quantitative performance criteria, and in particular met our target on domestic arrears older than 60 days by a large margin. We were able to reduce our primary deficit excluding grants from 5.1 percent of GDP in the first half of the year to 2.4 percent in the second half. Given the significant downward revision in projected revenues for 2009, we are proposing to revise the end-June 2009 target for the primary balance excluding grants.

Regarding the resolution of Capital Bank, the High Court has not yet acted on our petition for liquidation on November 13, 2008, pending several legal challenges by the bank's owner. Once the court has ruled on these challenges and on the liquidation petition, we will move rapidly to resolve the bank through liquidation.

We have also made significant progress with other structural reforms. We have announced an introduction date for the VAT of February 1, 2010, which will allow sufficient time for businesses, consumers, and the government to prepare, and we will soon begin our public education campaign. In addition, in January 2009 we established a Debt Management Unit in the Ministry of Finance, which aims at improving the effectiveness of and developing a strategy for our debt management. 
We are considering seeking a US\$50-80 million (7-12 percent of GDP) concessional loan from the Export-Import Bank of China to build a luxury hotel in a joint venture, although this loan is not accommodated under our program ceiling on bilateral concessional debt. We have given no further consideration to a loan for a marina and port project. We have asked the Paris Club to grant an extension of debt relief for 2009 in line with the debt relief that the Paris Club extended in 2006-08. We have also maintained our best efforts to complete the restructuring of our bilateral debt. We will continue good faith efforts to reach collaborative agreements with the few remaining nonparticipating commercial creditors.

Even with our ongoing efforts explained in detail in the attached SMEP, the global downturn is expected to have large adverse effects on economic activity and the external position of Grenada in 2009. To support our adjustment, we request an augmentation of access under the PRGF-supported program of 37.5 percent of quota, corresponding to SDR 4.39 million, to be disbursed at the conclusion of the third and fourth reviews.

The attached SMEP presents our policies for 2009 and beyond. Proposed quantitative performance criteria and indicative targets are indicated in Table 1 of the SMEP, and proposed structural benchmarks are indicated in Table 2 . We are committed to working to achieve these program targets, including the fiscal targets. On this basis, the Government of Grenada hereby requests the completion of the third review under the PRGF arrangement and financing assurances review and the release of the associated disbursement in an amount equivalent to SDR 3.875 million.

The government will continue to provide the Fund with such information as the Fund may request in connection with progress in implementing the economic and financial policies. The government believes that the policies and measures set forth in our previous MEP and the attached SMEP will achieve the program's objectives. The government also stands ready to take additional policy measures as appropriate to ensure the attainment of these objectives. We will consult with the Fund on the adoption of these measures, and in advance of revisions to the policies contained in the MEP and SMEP, in accordance with the Fund's policies on such consultations.

The government authorizes the Fund to make public the contents of this letter and the attached SMEP, as well as the accompanying staff report, to facilitate wider access to our policies and to signal the seriousness of our commitment to the program to civil society and the international community.

Yours faithfully, $/ \mathrm{s} /$

Honorable V. Nazim Burke

Minister for Finance 


\section{Attachment II: Supplementary Memorandum of Economic Policies}

1. Grenada's economic strategy is focused on coping with external shocks while laying the groundwork for broad-based economic growth. Achieving these goals will require implementing policies to address the impact of the global economic slowdown and financial spillovers; putting public finances and debt on a sustainable trajectory; improving the climate for private investment; reducing vulnerabilities; and alleviating poverty through more effective social safety nets. This supplement to our Memorandum of Economic Policies dated March 21, 2006 sets forth these economic objectives in greater detail and our plan for achieving them.

\section{RECENT DEVELOPMENTS AND OUTLOOK}

2. Economic growth has decelerated sharply reflecting the global slowdown. Real GDP growth slowed to 0.3 percent in 2008, from 4.5 percent in 2007, reflecting weakening tourism receipts and FDI. Inflation fell to 2.8 percent by March 2009, after peaking at 9.3 percent in July 2008, reflecting the passthrough of declining world fuel and food prices.

3. External conditions are placing pressure on the balance of payments. Stayover arrivals declined by 5 percent in 2008 after strong Cricket World Cup-related performance in 2007 , and the decline is expected to be more pronounced in 2009. Weaker tourism receipts and FDI turned the overall balance of payments into deficit in 2008.

4. Looking forward, real GDP is now expected to decline by 0.7 percent in 2009 , as a result of a deeper and more prolonged global slowdown. Stayover arrivals are expected to decline further, by about 10 percent, and private remittances are also expected to decline. The current account balance will narrow sharply as tourism receipts and FDI continue to fall, while the overall balance is projected to deteriorate further.

5. The banking sector, which is dominated by subsidiaries of international banks, has continued to remain resilient notwithstanding the global financial turmoil. Private sector credit growth slowed to 9.3 percent at end-February, reflecting the weaker economic activity. As at end-December 2008, the ratio of nonperforming loans to total loans remained low at 3.5 percent. However, banks report increases in the ratio in 2009, as some nonresident and resident borrowers are experiencing repayment difficulties.

6. The financial difficulties of the Trinidad and Tobago-based CL Financial Group are creating significant uncertainties. The group has a number of linkages to the Grenadian economy, significantly through the presence of insurance subsidiaries which have been offering deposit-like products. Available information suggests that Grenada does not have any direct exposure to the Stanford Financial Group.

\section{Program Performance}

7. We met all of our end-2008 quantitative program targets. We were able to reduce our primary deficit excluding grants (measured above-the-line) from EC $\$ 88$ million (5.1 percent of GDP) in the first half of the year to EC $\$ 41$ million (2.4 percent of GDP) in 
the second half. The improvement was a reflection of the strong measures we put in place in the second half of 2008, including a finance circular issued in September 2008 to limit capital expenditure, bringing forward the date after which expenditure commitments for nonessential expenditures could not be made, and reinstating the EC $\$ 3$ per gallon fuel tax and the automatic fuel price mechanism in October 2008.

- Capital expenditure of EC\$215 million (12.5 percent of GDP) in 2008 was higher than the programmed EC\$184 million (10.7 percent of GDP). Nevertheless, we were able to reduce capital expenditure by 3 percentage points of GDP in the second half of the year.

- We achieved a current surplus of 2.4 percent of GDP (above the line) for 2008, higher than the program target of 1.8 percent of GDP. The second half-year revenue outturn exceeded projections by 0.6 percent of GDP.

- The overall balance narrowed sharply (below the line) in the second half of the year. We lowered expenditure arrears from EC\$33 million (1.9 percent of GDP) at end-June 2008 to EC\$9.7 million (0.6 percent of GDP) at end-2008. We also rolled over late principal payments on treasury bills. Together, this reduced arrears older than 60 days to only EC $\$ 8.4$ million ( 0.5 percent of GDP), meeting this performance criterion by a significant margin.

8. We have made significant progress with structural reforms, but the implementation of some of our structural benchmarks for the third review will be delayed.

- We petitioned the High Court for liquidation of Capital Bank on November 13, 2008 (performance criterion), acting on the recommendation of the receiver and following consultation with the ECCB. However, the High Court has not yet acted on our petition pending several legal challenges by the bank's owner.

- $\quad$ We established a Debt Management Unit in the Ministry of Finance in January 2009 (structural benchmark), which we expect will improve the quality of our debt management and our capacity to critically assess proposals for further borrowing.

- We have announced an introduction date for the VAT of February 1, 2010. We have set up all implementation committees and finalized policy issues, and are beginning the public education campaign, and working on updating the tax administration information system. We submitted the VAT Bill to Parliament in April 2009, and expect to submit the new Excise Bill in August 2009 (proposed structural benchmark).

- $\quad$ The Office of Private Sector Development has spearheaded the work of a multisectoral Investment Policy Review Committee on the new draft Investment Act. The IMF Legal Department and the International Finance Corporation (IFC) provided technical assistance on the legislation, which will provide a transparent and level regime for domestic and foreign investors and replace tax holidays with investment 
allowances. We plan to submit the new Investment Act, the amended Income Tax Act, and repeal of tax incentives to Parliament by end-May 2009 (structural benchmark).

As a result of delays in completing the technical work and report compilation, we are proposing to push back the expected completion date for the Country Poverty Assessment until August 2009 (proposed structural benchmark).

\section{POLICIES FOR 2009}

9. We remain committed to strong, sound economic and fiscal management. Our policy framework is aimed at reducing our high public debt levels, primarily through fiscal consolidation. Addressing the adverse effects of the global economic slowdown is a near-term priority. Enhancing the business and investment environment, in partnership with the private sector, is a key objective. A major restructuring in the Ministry of Finance to support these objectives is underway, which has included establishment of a Waste Reduction Unit, a Division of Economic Planning and Management, and, as noted above a Debt Management Unit and an Office of Private Sector Development.

\section{To address the impact of the global slowdown on our economy, we will} accelerate capital spending programs that provide the greatest stimulus to economic activity and will undertake well-targeted spending to protect vulnerable groups. A set of priority projects, approved by Cabinet, has been selected, based on the criteria of highest employment generation potential, equitable geographic distribution, economic visibility to boost confidence and availability of funding. However, given the more constrained financing outlook, our revised target for capital expenditure will be EC $\$ 160$ million (8.9 percent of GDP). The public assistance program will be expanded, and a new road maintenance and debushing program (EC \$4 million), which will provide a direct safety net for poor households, will be implemented in August and December 2009. Other recurrent expenditure would remain within budgeted levels.

\section{We are considering special temporary measures to help the tourism sector} weather the adverse external shock. This could include an additional allocation of EC\$10 million to the Ministry of Tourism for marketing and airline support, and would be partly offset by expenditure savings elsewhere. We are also considering some temporary reductions in taxes on hotels' food imports, which would have a limited fiscal impact.

\section{Total revenue and grants for $\mathbf{2 0 0 9}$ are now projected to be lower than budgeted} as a result of the changed global environment. The sharp decline in imports has already reduced revenue from import-related taxes. In addition, PetroCaribe grants are projected to fall sharply in 2009, reflecting lower world fuel prices, the lower share of the fuel import bill which is financed concessionally, and our decision to transfer 35 percent rather than 65 percent of the amounts financed to the budget as grants. 
13. To achieve our fiscal objectives for 2009 , we plan to undertake the following fiscal measures:

- Maintaining the specific fuel tax at EC\$3 per gallon and continuing application of the automatic fuel price adjustment mechanism.

- Intensifying the successful program of collecting tax and nontax arrears and enhancing use of tax enforcement measures including garnishing income and seizing assets, following the completion of the tax amnesty, which yielded EC \$7.5 million ( 0.4 percent of GDP). The tax amnesty was extended from end-March to end-April only for taxpayers who had already applied by the end of March. We have also reestablished the Tax Tribunal, a specialized court to hear tax cases.

- $\quad$ Freezing wages for public service workers in 2009, except for the police, for whom an agreement had been completed in 2008 .

- $\quad$ Targeting capital expenditure of EC $\$ 160$ million (8.9 percent of GDP), and minimizing use of special warrants. Capital spending will, however, be slightly frontloaded in the first half of the year, in order to accelerate the stimulus to economic activity.

- $\quad$ Reducing spending on goods and services to EC\$74 million (4.1 percent of GDP), through bulk procurement internally and in cooperation with other ECCU members. The new Waste Reduction Unit in the Ministry of Finance is expected to achieve savings on fuel for government vehicles, fleet insurance and electricity costs.

- $\quad$ Reviewing eligibility for public assistance, while increasing the monthly transfer from EC $\$ 150$ to EC $\$ 200$ per month and accommodating increased demand for assistance in the difficult economic environment. This would entail a net increase in transfers and subsidies of EC $\$ 2$ million ( 0.1 percent of GDP).

14. Nevertheless, given the significant downward revision in projected revenues, it will not be possible to meet our target for end-June 2009 through deeper cuts in capital expenditure. Further cuts would jeopardize our medium-term growth objectives and the social consensus for reform. Therefore, we propose to revise the target for the primary deficit (excluding grants) for end-June 2009. The targeted deficit would be increased from EC\$28 million (1.6 percent of GDP) to EC\$53 million (2.9 percent of GDP). We are committed to meeting our revised program target and will take offsetting measures as needed in case of further shortfalls in revenue. We are also proposing an end-November 2009 target of EC\$78 million (4.3 percent of GDP) in line with this framework.

15. We plan to keep the stock of domestic arrears older than 60 days close to the low level achieved at end-2008. These arrears declined from EC $\$ 29$ million (1.7 percent of GDP) at end-September 2008 to EC $\$ 8.4$ million (0.5 percent of GDP) at end-2008. 
16. We have decided to increase the frequency of adjustments in retail fuel prices from an eight-week to a four-week cycle to reduce the magnitude of each adjustment. It will also limit periods of margin losses or gains by fuel importers which are ultimately borne by the budget. We have cleared arrears to fuel importers resulting from incomplete passthrough of world fuel prices in 2007-08 through an agreement with these importers under which we remitted back 50 percent of the fuel tax to reduce these claims. We do not expect to accumulate any further arrears to importers through consistent use of the automatic fuel price mechanism.

17. Our fiscal program for $\mathbf{2 0 0 9}$ is fully financed. Divestment proceeds from the Four Seasons hotel project, which would have been the main source of financing projected for 2009, will not be received this year in the adverse global environment for financing and tourism. We have requested that the Paris Club extend the debt relief that it extended in 2006-08 through 2009. We have also approached the World Bank and Caribbean Development Bank for budget support during 2009-10 seeking a high level of concessionality to support our adjustment to the external shock to tourism and foreign direct investment. In addition, we are negotiating an EC\$20 million (1.1 percent of GDP) loan from a consortium of commercial banks. We also intend to limit recourse to expensive sources of financing, such as the overdraft facility. We now expect to reach the 60 percent debt-to-GDP target by 2024, four years after the ECCU's 2020 benchmark for the region.

18. The government believes that the policies and measures set forth above will be sufficient to achieve our fiscal targets. We would undertake contingent measures if needed to reduce spending, including limiting grants and subventions while protecting vulnerable groups, and further rationalizing capital spending.

19. We are laying the foundation for a broadening of the tax base beginning in 2010. The VAT, which will be introduced in February 2010, is a broader and more efficient tax and, as in other ECCU countries, we would expect to see revenue gains of 0.5 to 1 percent of GDP. The VAT will have a 15 percent rate for goods and services, with a limited list of basic food items and chronic disease drugs zero-rated, and a 10 percent rate for hotel accommodation. We are also moving toward introduction of a market-based property tax. We completed the cadastral survey in December 2008, except in Carriacou, as a basis for reassessing the value of property. The new valuations would be applied in January 2011.

20. We intend to accelerate structural reforms focusing on tax and customs reform, economic management, and laying the groundwork for a comprehensive poverty reduction strategy.

- We will develop and begin implementing a customs Fraud Control Plan by August 2009. This will provide for risk profiles as a basis for risk-based inspections, in conjunction with the introduction of ASYCUDA World.

- $\quad$ A summary of key findings of the Country Poverty Assessment (CPA) was submitted to Cabinet in May 2009, and we expect to complete the assessment in August 2009. The CPA will provide information to help improve targeting of social programs. We 
will build on this assessment, together with our earlier National Development Strategy, to finalize a full PRSP by November 2009.

- $\quad$ Preparations to introduce a VAT are proceeding well. We have already begun efforts on public awareness and education. We plan to recruit and begin training staff and to adopt transitional procedures for bonded warehouses by May 2009. CARTAC is providing technical assistance on implementation, drawing on the successful experiences in other ECCU countries.

- We are developing an action plan to improve Doing Business Indicators, with technical assistance from the World Bank, to improve the business and investment environment. Five priority areas have been identified, and objectives determined: starting a business, trading across borders, paying taxes, registering property and enforcement of contracts. Appointment of a separate Registrar of Companies, an important component of a one-stop shop for business start-up, has been identified from the Action Plan as a structural benchmark for the fourth review (October 2009).

- We plan to establish a Public Procurement Authority by September 2009 as mandated by Parliament, in order to realize gains in efficiency, uniformity of procedures, and savings for government purchases. We intend to implement several pieces of public financial management legislation enacted in 2007-08, including the Integrity in Public Life, Prevention of Corruption, Public Finance Management, Audit, and Public Procurement and Contract Administration Acts. We will also work with the World Bank to establish bulk procurement procedures with another OECS country, extending the existing successful procedure with pharmaceuticals to other products such as textbooks, agricultural inputs, fleet insurance and tires.

21. The resolution of Capital Bank will proceed once the High Court has ruled on the legal challenges by the bank's owner. We petitioned the High Court for liquidation on November 13, 2008, following consultation with the ECCB. We have stated publicly that we do not intend to compensate depositors. We intend to proceed directly to liquidation as soon as the court permits.

\section{We are considering seeking a US\$50-80 million (7-12 percent of GDP)} concessional loan from the Export-Import Bank of China to build a luxury hotel in a joint venture with private investors. Currently this loan is not accommodated under our program ceiling on bilateral concessional debt through the end of 2009 . We may propose to revise upward our program's ceiling on bilateral concessional borrowing for a project that does not jeopardize debt sustainability and has significant net positive benefits. We have not given further consideration to a loan for a marina and port project.

\section{We are prudently managing the concessional financing from PetroCaribe. In} January 2009, we increased the share of PetroCaribe financing set aside in the special account to ensure sufficient funds for repayment from 35 to 65 percent, with the remainder transferred to the budget as grants. PetroCaribe Grenada Ltd. will begin repaying the longterm debt obligations to PDVSA in October 2009 when the two-year grace period on the first borrowing expires. 
24. We are continuing best efforts to conclude bilateral agreements with Paris Club (the Russian Federation) and non-Paris Club creditors. We will continue to seek an outof-court settlement with the Export-Import Bank of Taiwan Province of China, and pursue good faith efforts to reach a collaborative agreement with Grenada's external commercial creditors that did not participate in the 2005 debt exchange. We have cleared or restructured all but EC $\$ 1$ million arrears on unrestructured domestic debt to the nonbank public incurred in June 2007 and there are EC $\$ 1.7$ million arrears on 90-day treasury bills held by domestic commercial banks.

\section{Program Monitoring}

25. We are improving our capability to monitor economic developments and program performance. We have designated a Ministry of Finance official as the PRGF program coordinator, with responsibility for overseeing the monitoring of macroeconomic and structural program targets. We have put in place a system of monthly monitoring of domestic arrears and below-the-line financing of the central government overall balance, and will update this on a monthly basis.

\section{OTHER ISSUES}

\section{Reducing Financial Sector and Natural Disaster Vulnerabilities}

26. We intend to continue monitoring the impact of developments with the troubled Trinidad and Tobago-based CL Financial Group. We are determining the economy's exposure to the subsidiaries Clico Life Insurance and British American Insurance. An appropriate regional approach is being taken to assessing the liquidity and solvency positions of the companies, and to developing a resolution plan that will contribute to maintaining confidence in the financial system while limiting the costs for the governments.

\section{We are continuing with efforts to strengthen nonbank financial sector} supervision by the Grenada Authority for the Regulation of Financial Institutions (GARFIN). We are building its capacity while pursuing an ambitious agenda of enhancing legislation, producing regulations, and developing and implementing supervisory practices (reporting, offsite and onsite monitoring) for each type of institution. We submitted the Money Services and Insurance Acts to Parliament in March 2009, and will submit the Cooperative Societies Act in June 2009.

\section{We will continue to participate in the World Bank's Caribbean Catastrophic}

Risk Insurance Facility. In June 2007, we began purchasing parametric insurance that pays the government a predetermined amount in case of hurricane or earthquake. An IDA credit has financed Grenada's insurance premium in the first two years and half the premium for the next year. The CDB has agreed to provide support for the remaining half of the premium payments for the year beginning June 2009. 


\section{Fiscal transparency}

29. We are continuing our efforts to improve fiscal transparency. We will begin disseminating to the public quarterly information with a lag of one quarter on the overall fiscal situation and gross financing needs starting May 2009 and publishing information on public enterprise finances. In line with the new Public Finance Management Act of 2007, we will require public enterprises to submit audited financial statements within four months after the close of the financial year. We will continue to publish newly granted or extended tax concessions on a quarterly basis. 
Table 1. Grenada: Quantitative Performance Criteria and Indicative Targets, 2008-09

\begin{tabular}{|c|c|c|c|c|c|c|c|c|}
\hline & & & & \multicolumn{5}{|c|}{2009} \\
\hline & \multicolumn{3}{|c|}{ End-Dec. 2008} & \multirow{2}{*}{$\begin{array}{l}\text { End-March } \\
\text { Prog. 1/ }\end{array}$} & \multicolumn{2}{|c|}{ End-June } & \multirow{2}{*}{$\begin{array}{l}\text { End-Sept. } \\
\text { Proposed 1/ }\end{array}$} & \multirow{2}{*}{$\begin{array}{l}\text { End-Nov. } \\
\text { Proposed }\end{array}$} \\
\hline & Prog. & Adjusted & Actual & & Prog. & Prop. & & \\
\hline Performance Criteria: & \multicolumn{8}{|c|}{ (In millions of Eastern Caribbean dollars) } \\
\hline Central government primary balance excluding grants (floor) $2 / 3 /$ & -110.0 & -110.0 & -107.5 & -14.0 & -28.0 & -52.5 & -67.8 & -77.9 \\
\hline \multirow[t]{2}{*}{ Stock of central government domestic arrears (ceiling) } & 25.0 & 25.0 & 8.4 & 20.0 & 15.0 & 15.0 & 13.5 & 12.5 \\
\hline & \multicolumn{8}{|c|}{ (In millions of U.S. dollars) } \\
\hline $\begin{array}{l}\text { Contracting and guaranteeing of nonconcessional external debt by the central } \\
\text { government with maturity of at least one year (ceiling) } 2 /\end{array}$ & 4.0 & 4.0 & 0.0 & 4.0 & 4.0 & 4.0 & 4.0 & 4.0 \\
\hline Stock of external short term debt (ceiling) 4/ & 0.0 & 0.0 & 0.0 & 0.0 & 0.0 & 0.0 & 0.0 & 0.0 \\
\hline $\begin{array}{l}\text { Contracting and guaranteeing of bilateral concessional external debt by the } \\
\text { central government with maturity of at least one year (ceiling) } 2 / 5 /\end{array}$ & 0.0 & 0.0 & 0.0 & 0.0 & 0.0 & 0.0 & 0.0 & 0.0 \\
\hline Central government or guaranteed external arrears accumulation (ceiling) 4/ & 0.0 & 0.0 & 0.0 & 0.0 & 0.0 & 0.0 & 0.0 & 0.0 \\
\hline Indicative Target: & \multicolumn{8}{|c|}{ (In millions of Eastern Caribbean dollars) } \\
\hline Change in net credit of the banking system to the public sector (ceiling) $2 / 3 /$ & 24.0 & 45.0 & 16.9 & 0.0 & 0.0 & 26.9 & 31.9 & 35.2 \\
\hline $\begin{array}{l}\text { 1/ Indicative target. } \\
\text { 2/ Cumulative within each calendar year. } \\
\text { 3/ See the TMU and supplementary TMU for a description of adjustors. } \\
\text { 4/ To be monitored on a continuous basis. } \\
\text { 5/ Excludes PetroCaribe. }\end{array}$ & & & & & & & & \\
\hline
\end{tabular}


Table 2. Grenada: Structural Measures

\begin{tabular}{|c|c|c|c|}
\hline & Target Date & Comment & Macroeconomic Criticality \\
\hline \multicolumn{4}{|l|}{ Third Review } \\
\hline Reorganize or initiate liquidation of Capital Bank & November 2008 & Performance criterion. Met & To increase confidence in the financial system by having a clear bank resolution strategy \\
\hline $\begin{array}{l}\text { Submit to Parliament the Investment Act, the amended Income Tax Act, } \\
\text { and repeal of tax incentives }\end{array}$ & December 2008 & Benchmark. Not met & To improve the investment climate and reform the tax concessions regime \\
\hline Complete the Country Poverty Assessment & December 2008 & Benchmark. Not met & $\begin{array}{l}\text { To strengthen the ability of the government to develop effective and well-targeted poverty } \\
\text { reduction measures }\end{array}$ \\
\hline Establish a Debt Management Unit at the Ministry of Finance & February 2009 & Benchmark. Met & $\begin{array}{l}\text { To enable more effective debt management including better monitoring of payment } \\
\text { obligations and effective debt sustainability analysis }\end{array}$ \\
\hline Submit new VAT and Excise Bills to Parliament & April 2009 & Benchmark. Partially met & To increase the efficiency and effectiveness of revenue collection \\
\hline \multicolumn{4}{|l|}{ Fourth Review } \\
\hline $\begin{array}{l}\text { Recruit and begin training staff and adopt transitional procedures for } \\
\text { bonded warehouses }\end{array}$ & May 2009 & Benchmark & To ensure smooth implementation of the VAT \\
\hline Submit new Excise Bill to Parliament & August 2009 & Proposed benchmark & To increase the efficiency and effectiveness of revenue collection \\
\hline Complete the Country Poverty Assessment & August 2009 & Proposed benchmark & $\begin{array}{l}\text { To strengthen the ability of the government to develop effective and well-targeted poverty } \\
\text { reduction measures }\end{array}$ \\
\hline Develop and begin implementing a customs Fraud Control Plan & August 2009 & Benchmark & $\begin{array}{l}\text { To enhance customs administration and revenue collection for sustainable financing of } \\
\text { the budget }\end{array}$ \\
\hline Establish a Public Procurement Authority & September 2009 & Benchmark & To enhance transparency and governance in procurement and facilitate donor monitoring \\
\hline Appoint a separate Registrar of Companies & October 2009 & Proposed benchmark & To further improve the investment climate \\
\hline
\end{tabular}




\section{Attachment III: Supplementary Technical Memorandum of Understanding}

The Technical Memorandum of Understanding (TMU) associated with the LOI and MEP of March 21, 2006, as modified by the Supplemental TMUs of June 20, November 26, and December 9,2008, remains the operative document for monitoring and reporting requirements and for defining how the quantitative performance criteria and indicative targets, specified in Table 1 of the SMEP, will be interpreted, except as follows:

The end-June and end-November 2009 targets for the primary balance excluding grants and for net credit to the public sector will be revised downward and upward respectively by the amount of support to the insurance sector, with a maximum adjustment of EC $\$ 5$ million.

Substitute Table 1 in the TMU with the following table:

Table 1. Programmed Disbursements of Concessional Loans and Grants, 2009 (In millions of Eastern Caribbean dollars, cumulative)

\begin{tabular}{lrrrr}
\hline & \multicolumn{4}{c}{2009} \\
& $\begin{array}{r}\text { End-Mar. } \\
\text { Prel. }\end{array}$ & End-June & End-Sept. & End-Nov. \\
& 2.0 & 9.1 & 13.6 & 32.8 \\
\hline Concessional loans & 3.4 & 16.9 & 25.3 & 42.7 \\
Grants disbursements & 0.0 & 1.2 & 1.8 & 2.2 \\
Divestment proceeds & & &
\end{tabular}




\section{INTERNATIONAL MONETARY FUND}

\section{GRENADA}

\section{Debt Sustainability Analysis}

Prepared by the Staff of the International Monetary Fund

In consultation with the World Bank Staff

May 20, 2009

Grenada's outlook has deteriorated somewhat relative to the previous debt sustainability analysis (DSA), driven by the current economic downturn and higher debt financing. . Grenada will not achieve the regional benchmark of reducing the debt-to-GDP ratio to 60 percent until 2024, which is four years after the ECCB's target date of 2020 and a deterioration of six years relative to the previous DSA. A possible large loan from the Export-Import Bank of China to build a luxury hotel could undermine efforts to secure debt sustainability. Grenada exceeds thresholds for the ratios of the present value (PV) of external debt to GDP and to exports, and the ratio of the PV of external debt to GDP will fall below the 40 percent indicative threshold only in 2024. Thus, Grenada remains at high risk of debt distress. $^{2}$

\section{Context}

1. The global economic slowdown and financial turmoil have weakened Grenada's outlook significantly through their impact on tourism receipts, FDI, and remittances. Growth slowed from 4.5 percent in 2007 to 0.3 percent in 2008, and real GDP is projected to decline by 0.7 percent in 2009. Inflation, which stood at 2.8 percent as of March 2009, is expected to decline to 2.1 percent by the end of 2009, as lower world food and fuel prices are passed through.

2. Grenada obtained substantial debt relief through a commercial debt exchange in November 2005 and under a Paris Club agreement reached in May 2006. ${ }^{3}$ Nevertheless,

\footnotetext{
${ }^{1}$ See IMF Country Report No. 08/357.

2 Standard \& Poor's notes that Grenada's ratings are constrained by its high general government debt burden, debt management problems, and vulnerabilities inherent in the country's small size. It has not rated the sovereign in 2009.

${ }^{3}$ Bilateral agreements have been reached with all creditors except the Russian Federation.
} 
the debt service burden is now increasing reflecting the end of the grace period on restructured debt to domestic commercial banks, and the step up in the interest rate on exchanged debt from 1 percent during 2006-08 to 2.5 percent in 2009.

3. The government established a Debt Management Unit in the Ministry of Finance in January 2009 to improve the effectiveness of and to develop a strategy for debt management. The Unit is already making progress with plans to improve debt monitoring and the capacity to assess debt sustainability. It is also working to improve procedures to ensure timely debt service payments. ${ }^{4}$

\section{UNDERLYING DSA ASSUMPTIONS}

4. The baseline scenario is based upon the government's current fiscal policies, as set forth in their May 2009 Supplementary Memorandum of Economic Policies. ${ }^{5}$ The main assumptions under this scenario are described in the following table and in Box 1 .

Grenada: Key Assumptions and Indicators in the DSA, 2008-29

(In percent of GDP, unless otherwise indicated)

\begin{tabular}{|c|c|c|c|c|c|c|c|}
\hline & \multirow{2}{*}{$\begin{array}{r}\text { Prelim. } \\
2008 \\
\end{array}$} & \multicolumn{6}{|c|}{ Projections } \\
\hline & & 2009 & 2010 & 2011 & 2019 & 2025 & 2029 \\
\hline Total revenue and grants $1 /$ & 44.3 & 39.7 & 41.4 & 41.7 & 40.2 & 39.7 & 39.5 \\
\hline Primary (noninterest) expenditure $1 /$ & 46.5 & 41.3 & 41.1 & 39.2 & 38.0 & 37.3 & 36.8 \\
\hline Primary balance (including grants) $1 /$ & -2.3 & -1.6 & 0.3 & 2.5 & 2.2 & 2.5 & 2.7 \\
\hline Overall balance $1 /$ & -5.7 & -4.9 & -2.7 & -0.7 & -1.6 & -0.6 & 0.3 \\
\hline Public debt & 108.6 & 109.4 & 108.9 & 104.6 & 72.0 & 55.3 & 43.0 \\
\hline External current account & -42.3 & -32.0 & -30.3 & -29.9 & -27.6 & -24.2 & -21.9 \\
\hline Exports of goods and services & 27.7 & 24.0 & 25.0 & 26.1 & 30.2 & 31.0 & 31.4 \\
\hline Real GDP growth (in percent) & 0.3 & -0.7 & 1.0 & 2.9 & 4.0 & 4.0 & 4.0 \\
\hline Inflation rate (average; in percent) & 8.0 & 2.3 & 2.9 & 2.6 & 2.0 & 2.0 & 2.0 \\
\hline
\end{tabular}

Sources: Ministry of Finance of Grenada; and Fund staff estimates and projections.

1/ Public sector.

\footnotetext{
${ }^{4}$ The authorities made several small external debt service payments in 2008 with a delay. There have also been several cases in which there were delays in rolling over treasury bills. In addition, the government incurred EC \$20.3 million arrears in June 2007 on unrestructured debt to the nonbank public; all but EC\$1 million has been cleared.

${ }^{5}$ The baseline program scenario assesses the implications of the authorities' program under the PRGF arrangement, which focuses on securing debt sustainability through fiscal consolidation and on implementing structural reforms. The accompanying staff report includes an extensive description of the authorities' program.
} 


\section{Box 1. Baseline Macroeconomic Assumptions (2009-29)}

- Real GDP is projected to decline by 0.7 percent in 2009 due to the global economic slowdown and financial turmoil and to recover slightly to 1 percent in 2010 . Growth is projected to average 3.5 percent during 2011-14, and return to its potential of 4 percent subsequently. The growth projection for 2009 reflects a 37 percent decline in FDI, a 14 percent drop in travel receipts, and a 10 percent drop in net remittances for 2009 , all of which start to recover in 2010. In the longer run, the projection assumes that the authorities undertake the structural reforms needed to sustain growth.

- After rising to 8.0 percent in 2008 due to increases in food and fuel prices, annual average inflation is projected to fall gradually to 2 percent by 2014, and remain at this level thereafter.

- The primary balance (including grants) of the public sector improves from an average deficit of 2.1 percent of GDP during 1999-2008 to a surplus of 2.7 percent by 2014. This primary surplus reflects wage restraint in the medium term and annual central government capital expenditure of 8.2 percent of GDP.

- Grants gradually decline from high post-hurricane levels to 1 percent of GDP by 2029, and the interest rate on new borrowing increases from 2.6 percent in 2010 to 5 percent for 2011-2029. As concessional financing tapers off, and with a commercial borrowing rate above the discount rate of 5 percent, the grant element of new borrowing turns negative in later years.

- The projection assumes that Grenada receives concessional financing under the PetroCaribe initiative during 2009-12 (paragraph 6 below), and that the grant element is used to finance programs already in the budget, replacing more expensive forms of financing.

- The external current account deficit is assumed to decline gradually from 42.3 percent of GDP in 2008 to about 27.4 percent of GDP by 2014. Ongoing adjustment of the economy (reflected in a growing share of tourism receipts beginning in 2010) would allow the current account deficit to average around 23 percent of GDP in the outer years. ${ }^{1}$ Exports of goods and services are assumed to average 27.2 percent of GDP in 2009-14and are projected to average 30.6 percent for the rest of the period. Most of the financing would be provided by FDI inflows, averaging 17.9 percent of GDP in 2009-14 and 15.1 percent of GDP in the outer years, as well as by official transfers and portfolio investment inflows, averaging close to 3.5 percent of GDP over the projection period.

\footnotetext{
${ }^{1}$ This current account deficit is consistent with estimates of current account norms for ECCU countries (see Emilio Pineda, Paul Cashin, and Yan Sun, Assessing Exchange Rate Competitiveness in the Eastern Caribbean Currency Union, WP/09/78).
} 


\section{Evaluation of Public Sector Debt Sustainability}

\section{Grenada's public debt as of end-2008}

5. Public sector debt fell to $\mathbf{1 0 8 . 6}$ percent of GDP during 2008 (Tables 1 and A1a). The debt-to-GDP ratio declined by 3 percentage points of GDP, reflecting nominal GDP growth of 5 percent and the use of divestment proceeds to finance most of the overall deficit. Nevertheless, the end-2008 ratio was higher than the previous DSA's projection of 106.5 percent of GDP, as a result of the higher than expected overall deficit. External debt was 73.0 percent of total debt at end 2008. The central government accounted for almost all of external debt (88.1 percent). Public sector debt in PV terms at 103.9 percent of GDP was only slightly lower than in nominal terms, notwithstanding the concessional element of multilateral and restructured official debt, reflecting the stepping up of the interest rate on restructured debt to 9 percent by 2019 .

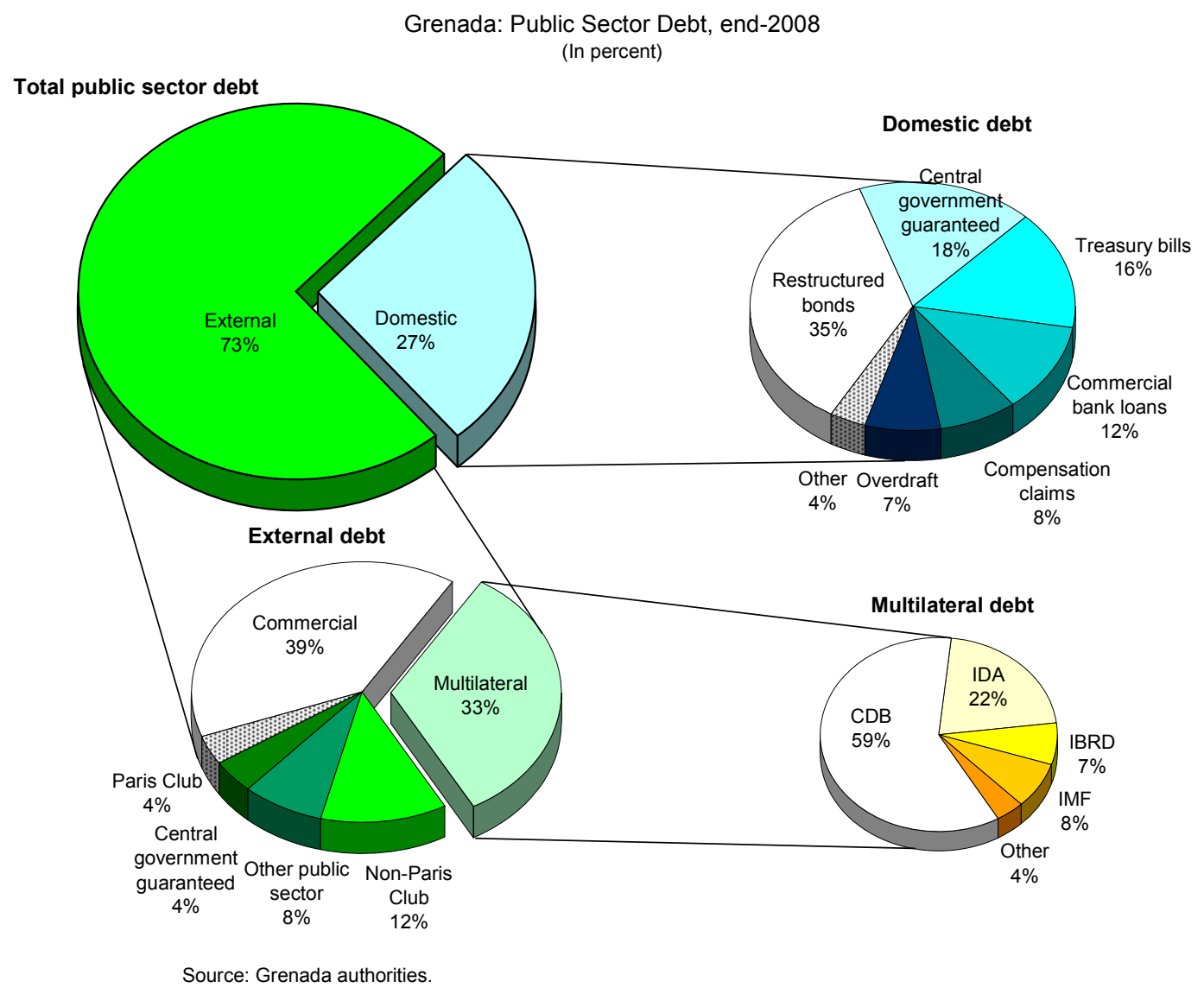




\section{The baseline (program) scenario}

\section{Grenada's debt sustainability outlook has deteriorated somewhat relative to the} previous DSA, notwithstanding expectations of greater concessional borrowing in the near term. ${ }^{6}$ The deterioration reflects the higher-than-expected debt at the end of 2008, the downward revision in the growth trajectory in the adverse external environment, and a higher overall deficit in 2009-10. Another important factor is that divestment proceeds from a luxury hotel project - the expected main financing source for the 2009 budget - will not materialize this year. Nevertheless, some of this deterioration is countered by lower

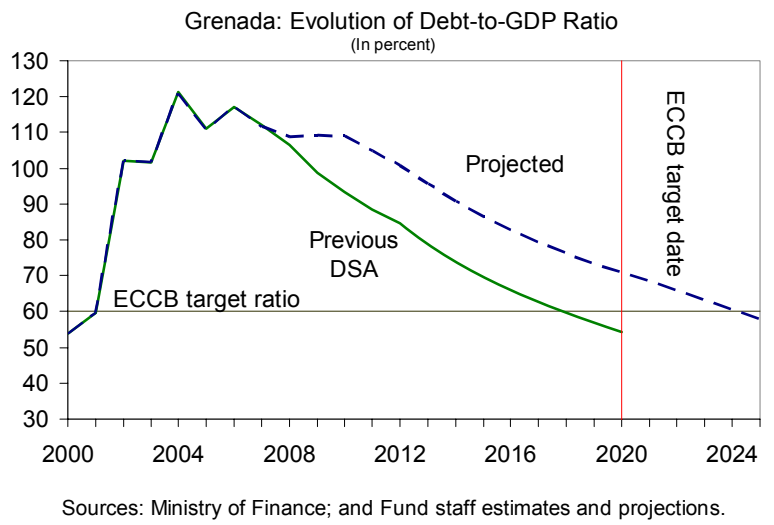
PetroCaribe borrowing, due to a lower expected path for oil prices in the medium term and higher primary surpluses in the long run (2015-29). ${ }^{7}$ The baseline does not include a proposed loan from the Export-Import Bank of China which is discussed below. ${ }^{8}$

\section{On the worsened debt trajectory, the authorities will not achieve the ECCU's} nominal debt target of 60 percent of GDP by 2020 until 2024, six years later than in the previous DSA. The PV of debt falls to 42.4 percent of GDP by 2029 , compared with 43.0 percent of GDP in nominal terms, reflecting new borrowing at a rate close to the discount rate of 5 percent (Figure 1). The PV of public sector debt-to-revenue ratio falls from 234.7 percent in 2008 to 107.4 percent in $2029 .^{9}$ The debt service-to-revenue ratio would increase from 12.5 percent in 2008 to 19.6 percent in 2029 , with the ratio rising to 27-28percent during 2021-25 as restructured debt amortizes.

\footnotetext{
${ }^{6}$ The projections assume an augmentation of the PRGF program (37.5 percent of quota or US\$6.5 million), and budgetary support from the Caribbean Development Bank (US\$12 million) and the World Bank (US\$8 million). In the long term, most of Grenada's new borrowing is assumed to be on commercial terms with an interest rate above the discount rate.

${ }^{7}$ The financing terms for PetroCaribe are highly concessional, with an interest rate of 1 percent and a maturity of 25 years (including a two-year grace period), implying a grant element of 46 percent. The agreement extends for a 14-year period, but is assumed to operate during 2008-12 reflecting the possibility that committed concessional financing might not be delivered.

${ }^{8}$ The program ceiling on bilateral concessional debt does not accommodate this loan.

${ }^{9}$ These ratios are stated with revenue inclusive of grants, consistent with Table A1a.
} 


\section{Alternative scenarios and stress tests}

\section{Policy scenarios}

8. The debt trajectory is highly sensitive to shocks and policy slippages. The following scenarios explore the potential impact of a delayed economic recovery, a lack of fiscal adjustment, or proposed borrowing from the Export-Import Bank of China.

\section{A scenario in which economic recovery is delayed due to a deeper and more prolonged global recession would be a setback to debt sustainability. This scenario} assumes a more protracted global economic downturn, with an 0.7 percent decline in real GDP in 2010-11 due to weak tourism sector performance. It also assumes a delayed recovery in FDI flows as a share of GDP as a result of continued difficulties in financing (Table A1b, Alternative Scenario A4). ${ }^{10}$ The PV of debt to GDP would fall to 45.6 percent by 2029 (46.2 percent in nominal terms), rather than 42.4 percent in the baseline scenario (Figure 1a). The 60 percent of GDP target for nominal debt is reached only in 2025 rather than 2024 in the baseline. The PV of debt to revenue would fall to 114.7 percent in 2029 , rather than 107.4 percent in the baseline scenario.

10. Fiscal adjustment is essential to reducing the level of debt. Under a scenario in which the primary fiscal balance remains unchanged at the 2009 level throughout the projection period, the nominal debt to GDP remains nearly constant (Table A1b, Alternative Scenario A2). With an unchanged primary balance, the PV of debt-to-GDP ratio reaches 103.6 percent by 2029 (109.3 percent in nominal terms).

\section{The authorities are considering a large proposed loan from the Export-Import} Bank of China in 2009-11 to finance a luxury hotel. Such a large loan could undermine the objective of reducing debt. Although the size and terms of the loan have yet to be fully finalized, this scenario assumes a loan amount of US\$80 million disbursed over 2009-11 with a 39 percent grant element, no private co-financing, and no impact on growth. ${ }^{11}$ With these assumptions, the average PV of debt-to-GDP ratio in 2009-14 is 104.6 percent (as opposed to 98.1 percent in the baseline scenario). In 2029, the PV of debt-to-GDP ratio is

\footnotetext{
${ }^{10}$ This is a similar scenario to the one with a natural disaster in the previous DSA which assumed that real GDP growth was zero for three years (2009-11) and then rose above the baseline during the recovery (2012-13), while the primary deficit was 3 percentage points of GDP higher during 2009-11. In that scenario, the PV of debt was 14 percentage points of GDP higher than in the baseline in 2028. A recent study has shown that Grenada's high vulnerability to natural disasters could potentially reverse the direction of a declining debt trajectory and has estimated that the projected baseline central government debt ratio without hurricanes could be between 29 to 129 percent of GDP by 2020 while with the impact of hurricanes this range would widen to 32-145 percent of GDP. See "Chapter VI. Insurance Against Natural Disasters in the Caribbean" in ECCUSelected Issues (IMF Country Report No. 09/176).

${ }^{11}$ This reflects the most recent information available to staff.
} 
46.0 percent (47.8 percent in nominal terms; Table A1b, Alternative Scenario A5). The 60 percent of GDP target for nominal debt is reached only in 2026 rather than 2024 in the baseline. If the project raises GDP growth by 0.5 percentage points per year during 2009-13, the PV of debt-to-GDP ratio in 2029 is 44.5 percent (46.1 percent in nominal terms), and the 60 percent of GDP target is reached in 2025 .

\section{Other scenarios}

12. It would be essential to contain expenditure if the downturn is prolonged. In Bound Test B1, real GDP contracts by 2.7 percent in 2010 and 2011, the fiscal revenue-toGDP ratio and nominal expenditure follow the same trajectories as in the baseline. In this scenario, the PV of debt rises sharply to 103.1 percent of GDP (108.4 percent in nominal terms) in 2029. In Bound Test B4, a 30 percent real depreciation in 2010 implies that the PV of debt falls only to 73.4 percent of GDP by 2029. In neither case would the 60 percent of GDP target for nominal debt be achieved in the projection period

\section{Evaluation of External Debt Sustainability}

13. Grenada continues to exceed thresholds for the ratio of the PV of external debt to GDP and to exports of goods and services. ${ }^{12}$ Based on Grenada's 2005-07 average CPIA rating of "medium," the thresholds are 40 percent and 150 percent for the ratios to GDP and exports, respectively, while for 2008, Grenada's ratios were 74.5 percent and 269.2 percent, respectively. ${ }^{13}$

14. However, Grenada is expected to remain above the threshold for the ratio for the PV of external debt to GDP for an extended period (Table A2a). The ratio of the PV of external public debt to GDP would not fall below the 40 percent threshold until 2023, while the PV of debt to exports would not fall below the 150 percent threshold until 2021 (Figure 2). The debt-service-to-exports ratio during 2009-14 remains well below the threshold for all but one year (2012), but breaches the 20 percent threshold in 2016 and remains above it for the remainder of the projection period..$^{14}$

15. A large possible loan from the Export-Import Bank of China would worsen the debt outlook. The PV of external public debt to GDP would not fall below the 40 percent

\footnotetext{
${ }^{12}$ Goods exports for Grenada are relatively small, reflecting hurricane damage during 2004-05 to the nutmeg industry.

${ }^{13}$ See Staff Guidance Note on the Application of the Joint Fund-Bank Debt Sustainability Framework for Low-Income Countries (Public Information Notice (PIN) No. 05/59 (May 4, 2005)).

${ }^{14}$ The analysis excludes flows of private sector external debt, which are believed to be small, due to lack of data. Given this exclusion, the size of the residuals in the baseline scenario is determined by developments in Grenada's BOP, e.g., the financing gap, the change in imputed reserves, and other investments (net).
} 
threshold until 2025, while the PV of debt to exports would fall below the 150 percent threshold in 2023, two years later than under the baseline.

16. Sensitivity analysis shows greater variation relative to the baseline in the short and medium term, but less variation in outer years (Table A2b). Under an alternative scenario with key variables (GDP growth, the current account deficit, and FDI) at historical averages, the PV of external public debt-to-GDP ratio would fall to 22.6 percent by 2029 (Scenario A1), slightly lower than in the baseline scenario (see Figure 2) ${ }^{15}$ However if new public sector loans are made on less favorable terms during 2009-2029 (Scenario A2, assuming 2 percentage points higher interest rate than in the baseline), the PV of external debt to GDP would not fall below the 40 percent threshold until 2028, and both the PV of debt-to-exports ratio, and debt-service-to-exports ratio will remain above the thresholds during most of this period.

\section{Conclusions}

17. Grenada's debt sustainability has deteriorated owing to lower economic growth, worsened primary deficits, and lower FDI flows due to the global economic slowdown. The authorities have responded prudently by prioritizing spending plans and enhancing efforts to protect vulnerable groups. The debt outlook is most sensitive to a protracted delay in fiscal adjustment, which would prevent the authorities from reaching the 60 percent of GDP debt target during the projection period. The outlook would also be adversely affected by the possible large loan from the Export-Import Bank of China and to any delays in economic recovery due to a deeper and more prolonged global downturn. Either of these shocks would significantly set back the goal of putting debt on a sustainable trajectory.

18. Grenada's risk of external debt distress remains high. External debt, although on a sustainable path, remains above the indicative thresholds for most of the period of the DSA analysis. In addition, the external debt outlook could deteriorate substantially if public sector loans were to be contracted on less favorable terms, thus calling for the authorities' careful consideration of the terms of debt contracted in the future.

\footnotetext{
${ }^{15}$ The historical average real GDP growth rate, which includes post-hurricane recovery years, is higher than average growth rate projected for the medium term in the baseline, which is driven by the effect of the global economic slowdown.
} 
Table 1. Grenada: Public Sector Debt, 2008

(Year end, in millions of U.S. dollars)

\begin{tabular}{|c|c|c|c|}
\hline & & Per & \\
\hline & Stock & Total Debt & GDP \\
\hline Public sector debt $1 /$ & 693.3 & 100.0 & 108.6 \\
\hline Central government debt & 600.3 & 86.6 & 94.0 \\
\hline Central-government guaranteed debt & 54.8 & 7.9 & 8.6 \\
\hline Other public sector debt & 38.2 & 5.5 & 6.0 \\
\hline External debt & 506.0 & 73.0 & 79.2 \\
\hline A. Central government & 445.8 & 64.3 & 69.8 \\
\hline 1. Multilateral & 167.1 & 24.1 & 26.2 \\
\hline CDB & 100.2 & 14.4 & 15.7 \\
\hline IDA & 36.0 & 5.2 & 5.6 \\
\hline IBRD & 11.5 & 1.7 & 1.8 \\
\hline IMF & 13.0 & 1.9 & 2.0 \\
\hline Other multilateral & 6.5 & 0.9 & 1.0 \\
\hline 2. Official bilateral & 79.3 & 11.4 & 12.4 \\
\hline Paris Club & 17.9 & 2.6 & 2.8 \\
\hline Belgium & 7.3 & 1.0 & 1.1 \\
\hline France & 4.3 & 0.6 & 0.7 \\
\hline Russian Federation & 0.2 & 0.0 & 0.0 \\
\hline United Kingdom & 3.3 & 0.5 & 0.5 \\
\hline United States & 2.9 & 0.4 & 0.5 \\
\hline Non-Paris Club & 61.4 & 8.9 & 9.6 \\
\hline Kuwait & 18.5 & 2.7 & 2.9 \\
\hline Taiwan Province of China & 20.3 & 2.9 & 3.2 \\
\hline Trinidad and Tobago & 17.1 & 2.5 & 2.7 \\
\hline Other bilateral & 5.6 & 0.8 & 0.9 \\
\hline 3. Commercial, total & 199.4 & 28.8 & 31.2 \\
\hline Restructured bonds & 193.5 & 27.9 & 30.3 \\
\hline Unrestructured bonds & 5.8 & 0.8 & 0.9 \\
\hline B. Central government guaranteed & 22.0 & 3.2 & 3.4 \\
\hline Of which: & & & \\
\hline Paris Club & 6.0 & 0.9 & 0.9 \\
\hline C. Other public sector $2 /$ & 38.2 & 5.5 & 6.0 \\
\hline Domestic debt & 187.3 & 27.0 & 29.3 \\
\hline A. Central government & 154.5 & 22.3 & 24.2 \\
\hline Restructured Bonds & 68.1 & 9.8 & 10.7 \\
\hline Unrestructured bonds & 2.8 & 0.4 & 0.4 \\
\hline Treasury bills & 29.1 & 4.2 & 4.6 \\
\hline Commercial bank loans & 21.8 & 3.1 & 3.4 \\
\hline Overdraft & 13.7 & 2.0 & 2.1 \\
\hline Domestic arrears & 3.6 & 0.5 & 0.6 \\
\hline Compensation claims & 14.8 & 2.1 & 2.3 \\
\hline Other & 0.6 & 0.1 & 0.1 \\
\hline B. Central-government guaranteed & 32.8 & 4.7 & 5.1 \\
\hline Memorandum item: & & & \\
\hline Nominal GDP & 638.5 & & \\
\hline
\end{tabular}

Sources: Grenada authorities; and IMF staff estimates.

1/ Includes central government liabilities to the National Insurance Scheme.

2/ Includes PetroCaribe-related borrowing. 
Table A1a. Grenada: Public Sector Debt Sustainability Framework, Baseline Scenario, 2006-29 1/ (In percent of GDP, unless otherwise indicated)

\begin{tabular}{|c|c|c|c|c|c|c|c|c|c|c|c|c|c|c|c|c|}
\hline & \multicolumn{3}{|c|}{ Actual } & \multirow[b]{2}{*}{$\begin{array}{c}1999-08 \\
\text { Average } \\
21 \\
\end{array}$} & \multirow[b]{2}{*}{$\begin{array}{c}\text { Standard } \\
\text { Deviation } \\
2 / \\
\end{array}$} & \multicolumn{11}{|c|}{ Projections } \\
\hline & 2006 & 2007 & 2008 & & & 2009 & 2010 & 2011 & 2012 & 2013 & 2014 & $\begin{array}{l}2009-14 \\
\text { Average }\end{array}$ & 2019 & 2025 & 2029 & $\begin{array}{l}2015-29 \\
\text { Average }\end{array}$ \\
\hline $\begin{array}{l}\text { Public sector debt } 1 / \\
\text { o/w foreign-currency denominated }\end{array}$ & $\begin{array}{r}117.3 \\
81.6\end{array}$ & $\begin{array}{r}111.5 \\
78.7\end{array}$ & $\begin{array}{r}108.6 \\
79.2\end{array}$ & & & $\begin{array}{r}109.4 \\
79.9\end{array}$ & $\begin{array}{r}108.9 \\
80.2\end{array}$ & $\begin{array}{r}104.6 \\
77.4\end{array}$ & $\begin{array}{r}100.5 \\
74.3\end{array}$ & $\begin{array}{l}95.1 \\
69.9\end{array}$ & $\begin{array}{l}90.2 \\
65.8\end{array}$ & & $\begin{array}{l}72.0 \\
50.6\end{array}$ & $\begin{array}{l}55.3 \\
36.0\end{array}$ & $\begin{array}{l}43.0 \\
24.9\end{array}$ & \\
\hline Change in public sector debt & 6.6 & -5.8 & -2.9 & & & 0.8 & -0.5 & -4.3 & -4.1 & -5.4 & -4.9 & & -2.9 & -2.9 & -3.1 & \\
\hline Identified debt-creating flows & 3.7 & -2.4 & -2.5 & & & -0.2 & -1.6 & -5.5 & -5.2 & -5.3 & -4.8 & & -2.9 & -2.9 & -3.1 & \\
\hline Primary deficit & 3.6 & 5.1 & 2.3 & 2.1 & 4.7 & 1.6 & -0.3 & -2.5 & -2.5 & -2.8 & -2.7 & -1.5 & -2.2 & -2.5 & -2.7 & 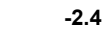 \\
\hline Revenue and grants & 44.6 & 39.2 & 44.3 & & & 39.7 & 41.4 & 41.7 & 41.5 & 41.3 & 41.2 & & 40.2 & 39.7 & 39.5 & \\
\hline of which: grants & 8.7 & 1.1 & 4.3 & & & 2.5 & 2.9 & 2.8 & 2.6 & 2.5 & 2.3 & & 1.7 & 1.2 & 1.0 & \\
\hline Primary (noninterest) expenditure & 48.1 & 44.3 & 46.5 & & & 41.3 & 41.1 & 39.2 & 39.0 & 38.6 & 38.5 & & 38.0 & 37.3 & 36.8 & \\
\hline Automatic debt dynamics & 0.7 & -5.4 & -1.9 & & & -1.6 & -1.0 & -2.8 & -2.5 & -2.4 & -1.9 & & -0.5 & -0.3 & -0.2 & \\
\hline Contribution from interest rate/growth differential & 1.6 & -5.1 & 0.0 & & & 1.4 & 0.0 & -2.0 & -2.0 & -2.1 & -1.8 & & -0.5 & -0.3 & -0.2 & \\
\hline of which: contribution from average real interest rate & -1.0 & -0.1 & 0.3 & & & 0.6 & 1.1 & 1.0 & 1.4 & 1.4 & 1.7 & & 2.4 & 2.0 & 1.6 & \\
\hline of which: contribution from real GDP growth & 2.7 & -5.0 & -0.3 & & & 0.8 & -1.1 & -3.1 & -3.4 & -3.5 & -3.6 & & -2.9 & -2.2 & -1.8 & \\
\hline Contribution from real exchange rate depreciation & -0.9 & -0.3 & -1.9 & & & -3.0 & -1.0 & -0.7 & -0.4 & -0.3 & -0.1 & & & & & \\
\hline Other identified debt-creating flows & -0.6 & -2.2 & -2.9 & & & -0.1 & -0.3 & -0.3 & -0.2 & -0.2 & -0.2 & & -0.2 & -0.1 & -0.1 & \\
\hline Privatization receipts (negative) & -0.6 & -2.2 & -2.9 & & & -0.1 & -0.3 & -0.3 & -0.2 & -0.2 & -0.2 & & -0.2 & -0.1 & -0.1 & \\
\hline Recognition of implicit or contingent liabilities & 0.0 & 0.0 & 0.0 & & & 0.0 & 0.0 & 0.0 & 0.0 & 0.0 & 0.0 & & 0.0 & 0.0 & 0.0 & \\
\hline Debt relief (HIPC and other) & 0.0 & 0.0 & 0.0 & & & 0.0 & 0.0 & 0.0 & 0.0 & 0.0 & 0.0 & & 0.0 & 0.0 & 0.0 & \\
\hline Other (specify, e.g. bank recapitalization) & 0.0 & 0.0 & 0.0 & & & 0.0 & 0.0 & 0.0 & 0.0 & 0.0 & 0.0 & & 0.0 & 0.0 & 0.0 & \\
\hline Residual, including asset changes & 2.9 & -3.4 & -0.4 & & & 1.0 & 1.1 & 1.2 & 1.1 & -0.1 & -0.1 & & -0.1 & -0.1 & 0.0 & \\
\hline \multicolumn{17}{|l|}{ Other Sustainability Indicators } \\
\hline PV of public sector debt & $\ldots$ & $\ldots$ & 103.9 & & & 105.1 & 105.0 & 101.0 & 97.2 & 92.4 & 87.9 & & 70.1 & 54.1 & 42.4 & \\
\hline $\mathrm{o} / \mathrm{w}$ foreign-currency denominated & $\ldots$ & $\ldots$ & 74.5 & & & 75.6 & 76.3 & 73.8 & 70.9 & 67.1 & 63.5 & & 48.7 & 34.8 & 24.3 & \\
\hline o/w external & $\ldots$ & $\ldots$ & 74.5 & & & 75.6 & 76.3 & 73.8 & 70.9 & 67.1 & 63.5 & & 48.7 & 34.8 & 24.3 & \\
\hline PV of contingent liabilities (not included in public sector debt) & & & & & & & & & & & & & & & & \\
\hline Gross financing need $3 /$ & 7.8 & 10.8 & 7.8 & & & 6.4 & 4.2 & 2.7 & 4.4 & 3.3 & 3.8 & & 5.3 & 8.1 & 5.0 & \\
\hline PV of public sector debt-to-revenue and grants ratio (in percent & $\ldots$ & $\ldots$ & 234.7 & & & 264.7 & 253.3 & 242.5 & 234.1 & 223.4 & 213.3 & & 174.2 & 136.1 & 107.4 & \\
\hline PV of public sector debt-to-revenue ratio (in percent) & $\ldots$ & $\ldots$ & 260.0 & & & 282.6 & 272.6 & 259.8 & 249.9 & 237.6 & 226.1 & & 182.1 & 140.3 & 110.1 & \\
\hline Of which: external $4 /$ & & & 186.6 & & & 203.3 & 198.0 & 189.9 & 182.5 & 172.7 & 163.4 & & 126.5 & 90.3 & 63.1 & \\
\hline Debt service-to-revenue and grants ratio (in percent) 5 / & 9.5 & 14.3 & 12.5 & & & 12.1 & 10.8 & 12.5 & 16.7 & 14.8 & 15.8 & & 18.8 & 26.7 & 19.6 & \\
\hline Debt service-to-revenue ratio (in percent) $5 /$ & 11.8 & 14.7 & 13.8 & & & 12.9 & 11.6 & 13.4 & 17.8 & 15.7 & 16.7 & & 19.6 & 27.5 & 20.1 & \\
\hline Primary deficit that stabilizes the debt-to-GDP ratio & -3.0 & 10.9 & 5.2 & & & 0.8 & 0.2 & 1.8 & 1.6 & 2.6 & 2.2 & & 0.7 & 0.4 & 0.4 & \\
\hline \multicolumn{17}{|l|}{ Key macroeconomic and fiscal assumptions } \\
\hline Real GDP growth (in percent) & -2.3 & 4.5 & 0.3 & 2.8 & 5.5 & -0.7 & 1.0 & 2.9 & 3.4 & 3.6 & 3.9 & 2.3 & 4.0 & 4.0 & 4.0 & 4.0 \\
\hline Average nominal interest rate on forex debt (in percent) & 2.8 & 2.9 & 3.3 & 5.3 & 2.9 & 3.0 & 2.9 & 3.3 & 3.5 & 3.5 & 3.9 & 3.3 & 5.4 & 5.7 & 5.7 & 5.4 \\
\hline Average real interest rate on domestic debt (in percent) & -2.1 & -0.7 & -1.5 & 1.3 & 2.8 & -1.7 & -0.1 & -0.1 & 0.9 & 1.2 & 2.0 & 0.4 & 3.3 & 3.3 & 3.3 & 3.2 \\
\hline Real exchange rate depreciation (in percent, + indicates depreciatior & -1.1 & -0.4 & -2.4 & -0.9 & 1.3 & & & & 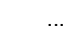 & & 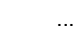 & 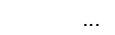 & $\ldots$ & & $\ldots$ & \\
\hline Inflation rate (GDP deflator, in percent) & 4.4 & 3.1 & 4.8 & 2.9 & 1.9 & 5.5 & 2.8 & 2.8 & 2.5 & 2.3 & 2.1 & 3.0 & 2.0 & 2.0 & 2.0 & 2.0 \\
\hline Growth of real primary spending (deflated by GDP deflator, in percer & 0.1 & 0.0 & 0.1 & 0.1 & 0.1 & -0.1 & 0.0 & 0.0 & 0.0 & 0.0 & 0.0 & 0.0 & 0.0 & 0.0 & 0.0 & 0.0 \\
\hline Grant element of new external borrowing (in percent) & $\ldots$ & . & $\ldots$ & $\ldots$ & $\ldots$ & 20.8 & 25.9 & 28.2 & 19.1 & 19.0 & 11.2 & 20.7 & -0.7 & -6.3 & -9.4 & \\
\hline
\end{tabular}

Sources: Grenada authorities; and IMF staff estimates and projections.

$1 /$ The coverage of the public sector is public and publicly guaranteed debt, including debt of public enterprises and the debt of the central government to the public sector.

2/ Historical averages and standard deviations are generally derived over the past 10 years, subject to data availability.

$3 /$ Gross financing need is defined as the primary deficit plus debt service plus the stock of short-term debt at the end of the last period.

$5 /$ Debt service is defined as the sum of interest and amortization of medium and long-term debt. 
Table A1b. Grenada: Sensitivity Analysis for Key Indicators of Public Debt 2009-29

\begin{tabular}{|c|c|c|c|c|c|c|c|c|c|}
\hline & \multicolumn{9}{|c|}{ Projections } \\
\hline & 2009 & 2010 & 2011 & 2012 & 2013 & 2014 & 2019 & 2025 & 2029 \\
\hline \multicolumn{10}{|c|}{ PV of Debt-to-GDP Ratio } \\
\hline Baseline & 105.1 & 105.0 & 101.0 & 97.2 & 92.4 & 87.9 & 70.1 & 54.1 & 42.4 \\
\hline \multicolumn{10}{|l|}{ A. Alternative scenarios } \\
\hline A1. Real GDP growth and primary balance are at historical averages & 105.1 & 105.2 & 105.4 & 106.1 & 106.3 & 107.0 & 113.1 & 123.4 & 129.2 \\
\hline A2. Primary balance is unchanged from 2009 & 105.1 & 106.7 & 106.3 & 106.1 & 105.1 & 104.2 & 102.7 & 103.8 & 103.6 \\
\hline A3. Permanently lower GDP growth 1/ & 105.1 & 106.7 & 104.7 & 103.2 & 101.0 & 99.5 & 102.0 & 123.1 & 143.6 \\
\hline A4. Delayed recovery & 109.7 & 114.1 & 115.5 & 111.1 & 105.7 & 100.4 & 78.5 & 59.0 & 45.6 \\
\hline A5. With China loan & 111.1 & 110.8 & 108.1 & 104.1 & 99.2 & 94.6 & 75.7 & 58.4 & 46.0 \\
\hline A6. With China loan with growth impact & 110.6 & 109.7 & 106.5 & 102.1 & 96.8 & 92.2 & 73.7 & 56.7 & 44.5 \\
\hline \multicolumn{10}{|l|}{ B. Bound tests } \\
\hline B1. Real GDP growth is at historical average minus one standard deviations in 2010-11 & 105.1 & 110.3 & 115.6 & 114.7 & 112.7 & 111.0 & 106.8 & 105.9 & 103.1 \\
\hline B2. Primary balance is at historical average minus one standard deviations in $2010-11$ & 105.1 & 111.3 & 115.5 & 111.4 & 106.4 & 101.7 & 83.0 & 65.5 & 52.9 \\
\hline B3. Combination of B1-B2 using one half standard deviation shocks & 105.1 & 110.2 & 115.3 & 112.5 & 108.7 & 105.1 & 92.0 & 80.9 & 72.1 \\
\hline B4. One-time 30 percent real depreciation in 2010 & 105.1 & 137.8 & 133.0 & 128.3 & 122.9 & 118.0 & 98.8 & 83.4 & 73.4 \\
\hline B5. 10 percent of GDP increase in other debt-creating flows in 2010 & 105.1 & 113.9 & 109.9 & 105.9 & 101.0 & 96.3 & 78.0 & 61.1 & 48.8 \\
\hline
\end{tabular}

PV of Debt-to-Revenue Ratio 2/

Baseline

$\begin{array}{lllllllll}264.7 & 253.3 & 242.5 & 234.1 & 223.4 & 213.3 & 174.2 & 136.1 & 107.4\end{array}$

\section{A. Alternative scenarios}

A1. Real GDP growth and primary balance are at historical averages A2. Primary balance is unchanged from 2009

A3. Permanently lower GDP growth $1 /$

A4. Delayed recovery

A5. With China loan

A6. With China loan with growth impact

\section{B. Bound tests}

B1. Real GDP growth is at historical average minus one standard deviations in 2010-11 B2. Primary balance is at historical average minus one standard deviations in 2010-11

B3. Combination of B1-B2 using one half standard deviation shocks

B4. One-time 30 percent real depreciation in 2010
B5. 10 percent of GDP increase in other debt-creating flows in 2010

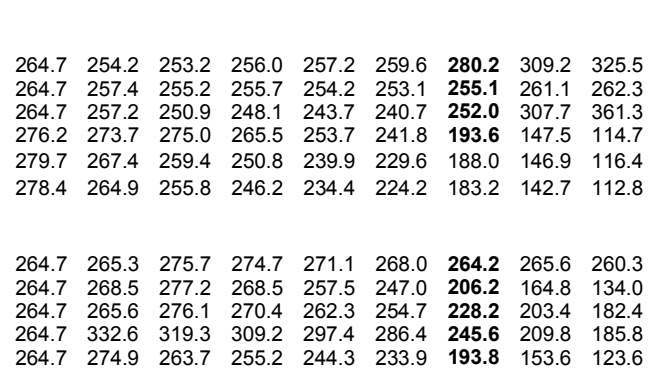

Debt Service-to-Revenue Ratio 2/

Baseline

$\begin{array}{lllllllll}12.1 & 10.8 & 12.5 & 16.7 & 14.8 & 15.8 & \mathbf{1 8 . 8} & 26.7 & 19.6\end{array}$

\section{A. Alternative scenarios}

A1. Real GDP growth and primary balance are at historical averages

A2. Primary balance is unchanged from 2009

A3. Permanently lower GDP growth $1 /$

A4. Delayed recovery

A5. With China loan

A6. With China loan with growth impact

B. Bound tests

B1. Real GDP growth is at historical average minus one standard deviations in 2010-11

B2. Primary balance is at historical average minus one standard deviations in 2010-11

B3. Combination of B1-B2 using one half standard deviation shocks

B4. One-time 30 percent real depreciation in 2010

B5. 10 percent of GDP increase in other debt-creating flows in 2010

Sources: Grenada authorities; and IMF staff estimates and projections.

1/ Assumes that real GDP growth is at baseline minus one standard deviation divided by the square root of the length of the projection period.

2/ Revenues are defined inclusive of grants. 
Table A2a. Grenada: External Debt Sustainability Framework, Baseline Scenario, 2006-29 1/

(In percent of GDP, unless otherwise indicated)

\begin{tabular}{|c|c|c|c|c|c|c|c|c|c|c|c|c|c|c|c|c|}
\hline & \multicolumn{3}{|c|}{ Actual } & \multirow{2}{*}{$\begin{array}{c}\text { Historical } \\
\text { Average 2/ }\end{array}$} & \multirow{2}{*}{$\begin{array}{c}\text { Standard } \\
\text { Deviation 2/ }\end{array}$} & \multicolumn{6}{|c|}{ Projections } & \multirow[b]{2}{*}{$\begin{array}{l}2009-14 \\
\text { Average }\end{array}$} & \multirow[b]{2}{*}{2019} & \multirow[b]{2}{*}{2025} & \multirow[b]{2}{*}{2029} & \multirow[b]{2}{*}{$\begin{array}{l}2015-29 \\
\text { Average } \\
\end{array}$} \\
\hline & 2006 & 2007 & 2008 & & & 2009 & 2010 & 2011 & 2012 & 2013 & 2014 & & & & & \\
\hline External debt (nominal) $1 /$ & 81.6 & 78.7 & 79.2 & & & 79.9 & 80.2 & 77.4 & 74.3 & 69.9 & 65.8 & & 50.6 & 36.0 & 24.9 & \\
\hline $\mathrm{o} / \mathrm{w}$ public and publicly guaranteed (PPG) & 81.6 & 78.7 & 79.2 & & & 79.9 & 80.2 & 77.4 & 74.3 & 69.9 & 65.8 & & 50.6 & 36.0 & 24.9 & \\
\hline Change in external debt & 2.3 & -2.9 & 0.5 & & & 0.6 & 0.3 & -2.8 & -3.1 & -4.4 & -4.1 & & -2.5 & -2.6 & -2.8 & \\
\hline Identified net debt-creating flows & 16.0 & 7.4 & 13.2 & & & 17.3 & 12.9 & 9.1 & 7.8 & 6.3 & 5.8 & & 10.6 & 7.7 & 5.9 & \\
\hline Non-interest current account deficit & 31.2 & 39.7 & 39.8 & 24.5 & 11.8 & 29.8 & 28.1 & 27.5 & 26.8 & 25.4 & 24.8 & & 24.9 & 22.1 & 20.4 & 23.1 \\
\hline Deficit in balance of goods and services & 41.7 & 41.2 & 43.2 & & & 31.4 & 30.1 & 29.3 & 28.3 & 26.9 & 26.1 & & 23.9 & 20.3 & 17.9 & \\
\hline Exports & 28.8 & 31.0 & 27.7 & & & 24.0 & 25.0 & 26.1 & 27.4 & 29.2 & 31.3 & & 30.2 & 31.0 & 31.4 & \\
\hline Imports & 70.4 & 72.2 & 70.9 & & & 55.4 & 55.1 & 55.4 & 55.7 & 56.0 & 57.4 & & 54.1 & 51.3 & 49.3 & \\
\hline Net current transfers (negative = inflow) & -13.9 & -5.0 & -6.8 & -11.8 & 7.0 & -4.6 & -4.9 & -4.6 & -4.4 & -4.2 & -4.0 & & -1.5 & -1.3 & -1.2 & -2.0 \\
\hline o/w official & -9.4 & -1.8 & -3.8 & & & -2.0 & -2.4 & -2.3 & -2.1 & -2.0 & -1.8 & & 0.5 & 0.4 & 0.4 & \\
\hline Other current account flows (negative = net inflow) & 3.4 & 3.5 & 3.4 & & & 3.0 & 2.9 & 2.8 & 2.9 & 2.7 & 2.7 & & 2.6 & 3.2 & 3.8 & \\
\hline Net FDI (negative = inflow) & -15.9 & -28.7 & -25.2 & -16.1 & 6.4 & -15.3 & -16.7 & -18.7 & -19.1 & -19.0 & -19.0 & & -15.0 & -15.0 & -15.0 & -15.1 \\
\hline Endogenous debt dynamics $3 /$ & 0.7 & -3.6 & -1.3 & & & 2.8 & 1.5 & 0.3 & 0.1 & -0.1 & 0.0 & & 0.7 & 0.6 & 0.5 & \\
\hline Contribution from nominal interest rate & 2.2 & 2.2 & 2.5 & & & 2.2 & 2.3 & 2.5 & 2.6 & 2.5 & 2.6 & & 2.7 & 2.1 & 1.5 & \\
\hline Contribution from real GDP growth & 1.8 & -3.4 & -0.2 & & & 0.5 & -0.8 & -2.2 & -2.5 & -2.5 & -2.6 & & -2.0 & -1.5 & -1.0 & \\
\hline Contribution from price and exchange rate changes & -3.3 & -2.5 & -3.6 & & & & & & & & -2.0 & & & & & \\
\hline Residual 4/ & -13.7 & -10.3 & -12.8 & & & -16.6 & -12.6 & -11.8 & -10.9 & -10.7 & -9.8 & & -13.1 & -10.4 & -8.7 & \\
\hline o/w exceptional financing & 0.0 & 0.0 & 0.0 & & & 0.0 & 0.0 & 0.0 & 0.0 & 0.0 & 0.0 & & 0.0 & 0.0 & 0.0 & \\
\hline $\begin{array}{l}\text { PV of external debt } 5 / \\
\text { In percent of exports }\end{array}$ & $\ldots$ & $\ldots$ & 74.5 & & & 75.6 & 76.3 & 73.8 & 70.9 & 67.1 & 63.5 & & 48.7 & 34.8 & 24.3 & \\
\hline $\begin{array}{l}\text { In percent of exports } \\
\text { PV of PPG external debt }\end{array}$ & $\ldots$ & & 269.2 & & & 315.7 & 304.7 & 283.1 & 259.2 & 230.0 & 203.0 & & 161.2 & 112.2 & 77.4 & \\
\hline $\begin{array}{l}\text { PV of PPG external debt } \\
\text { In percent of exports }\end{array}$ & $\ldots$ & $\ldots$ & $\begin{array}{r}74.5 \\
\end{array}$ & & & 75.6 & 76.3 & 73.8 & 70.9 & 67.1 & 63.5 & & 48.7 & 34.8 & 24.3 & \\
\hline $\begin{array}{l}\text { In percent of exports } \\
\text { In percent of governme }\end{array}$ & $\ldots$ & $\ldots$ & $\begin{array}{l}269.2 \\
1866\end{array}$ & & & 315.7 & 304.7 & 283.1 & 259.2 & 230.0 & 203.0 & & 161.2 & 112.2 & 77.4 & \\
\hline $\begin{array}{l}\text { In percent of government revenues } \\
\text { Debt service-to-exports ratio (in percent) }\end{array}$ & $\ldots$ & $\begin{array}{r}\ldots . \\
156\end{array}$ & $\begin{array}{r}186.6 \\
164\end{array}$ & & & 203.3 & 198.0 & 189.9 & 182.5 & 172.7 & 163.4 & & 126.5 & 90.3 & 63.1 & \\
\hline $\begin{array}{l}\text { Debt service-to-exports ratio (in percent) } \\
\text { PPG debt service-to-exports ratio (in percent) }\end{array}$ & $\begin{array}{l}12.4 \\
12.4\end{array}$ & $\begin{array}{l}15.6 \\
156\end{array}$ & $\begin{array}{l}16.4 \\
164\end{array}$ & & & $\begin{array}{l}15.7 \\
157\end{array}$ & $\begin{array}{l}14.8 \\
14.8\end{array}$ & $\begin{array}{l}17.1 \\
17.1\end{array}$ & 22.0 & 17.9 & 17.6 & & 21.3 & 31.0 & 21.7 & \\
\hline $\begin{array}{l}\text { PPG debt service-to-exports ratio (in percent) } \\
\text { PPG debt service-to-revenue ratio (in percent) }\end{array}$ & $\begin{array}{r}12.4 \\
9.9\end{array}$ & $\begin{array}{l}15.6 \\
12.7\end{array}$ & $\begin{array}{l}16.4 \\
11.3\end{array}$ & & & $\begin{array}{l}15.7 \\
10.1\end{array}$ & $\begin{array}{r}14.8 \\
9.6\end{array}$ & $\begin{array}{l}17.1 \\
111.5\end{array}$ & $\begin{array}{l}22.0 \\
15.5\end{array}$ & $\begin{array}{l}17.9 \\
13.4\end{array}$ & $\begin{array}{l}17.6 \\
14.2\end{array}$ & & $\begin{array}{l}21.3 \\
16.7\end{array}$ & $\begin{array}{l}31.0 \\
24.9\end{array}$ & $\begin{array}{l}21.7 \\
17.7\end{array}$ & \\
\hline Total gross financing need (Millions of U.S. dollars) & 106.5 & 96.2 & 121.9 & & & 121.9 & 104.9 & 97.4 & 107.0 & 95.4 & $\begin{array}{r}17.2 \\
98.8\end{array}$ & & $\begin{array}{l}16.7 \\
192.6\end{array}$ & $\begin{array}{r}24.9 \\
280.5\end{array}$ & 259.9 & \\
\hline Non-interest current account deficit that stabilizes debt ratio & 28.9 & 42.5 & 39.3 & & & 29.2 & 27.8 & 30.2 & 29.9 & 29.8 & 28.9 & & 27.4 & 24.8 & 23.2 & \\
\hline \multicolumn{17}{|l|}{ Key macroeconomic assumptions } \\
\hline Real GDP growth (in percent) & -2.3 & 4.5 & 0.3 & 2.8 & 5.5 & -0.7 & 1.0 & 2.9 & 3.4 & 3.6 & 3.9 & 2.3 & 4.0 & 4.0 & 4.0 & 4.0 \\
\hline GDP deflator in U.S. dollar terms (change in percent) & 4.4 & 3.1 & 4.8 & 2.9 & 1.9 & 5.5 & 2.8 & 2.8 & 2.5 & 2.3 & 2.1 & 3.0 & 2.0 & 2.0 & 2.0 & 2.0 \\
\hline Effective interest rate (percent) $6 /$ & 2.8 & 2.9 & 3.3 & 5.3 & 2.9 & 3.0 & 2.9 & 3.3 & 3.5 & 3.5 & 3.9 & 3.3 & 5.4 & 5.7 & 5.7 & 5.4 \\
\hline Growth of exports of G\&S (U.S. dollar terms, it & 9.0 & 15.9 & -6.0 & 2.1 & 17.1 & -9.4 & 8.5 & 10.2 & 11.2 & 13.0 & 13.7 & 7.9 & 6.8 & 6.4 & 6.4 & 6.1 \\
\hline Growth of imports of G\&S (U.S. & 0.6 & 10.4 & 3.2 & 6.4 & 9.9 & -18.2 & 3.3 & 6.4 & 6.5 & 6.7 & 8.7 & 2.2 & 5.2 & 5.1 & 4.9 & 5.0 \\
\hline Grant element $\mathrm{c}$ & & & & & $\ldots$ & 20.8 & 25.9 & 28.2 & 19.1 & 19.0 & 11.2 & 20.7 & -0.7 & -6.3 & -9.4 & -3.3 \\
\hline Government revenues (excluding & 35.9 & 38.1 & 39.9 & & & 37.2 & 38.5 & 38.9 & 38.9 & 38.9 & 38.9 & & 38.5 & 38.5 & 38.5 & 38.5 \\
\hline Aid flows (in Millions of U.S. dollar & 49.0 & 6.4 & 27.5 & & & 16.9 & 20.4 & 20.4 & 20.4 & 20.4 & 20 & & 20.4 & 20.4 & 20.4 & \\
\hline o/w G & 49.0 & 6.4 & 27.5 & & & 16.9 & 20.4 & 20.4 & 20.4 & 20.4 & 20.4 & & 204 & 204 & 204 & \\
\hline Grant-equivalent financing (in percent of GDP) 8/ & $\ldots$ & & $\ldots$ & & & 3.7 & 4.2 & 3.8 & 3.5 & 2.9 & 2.7 & & 1.7 & 0.8 & 0.6 & 1.3 \\
\hline Grant-equivalent financing (in percent of external financing) $8 /$ & $\ldots$ & $\ldots$ & $\ldots$ & & & 45.0 & 54.4 & 59.3 & 48.0 & 59.0 & 50.7 & & 28.2 & 9.2 & 11.2 & 20.4 \\
\hline \\
\hline Nomin & 564.4 & 607.9 & 638.5 & & & 668.8 & 694.4 & 734.6 & 778.6 & 825.1 & 875.3 & & 1175.8 & 1675.4 & 2121.6 & \\
\hline Nominal dol & 1.9 & 7.7 & 5.0 & & & 4.8 & 3.8 & 5.8 & 6.0 & 6.0 & 6.1 & 5.4 & 6.1 & 6.1 & 6.1 & 6.1 \\
\hline PV of PPG external & $\ldots$ & & 475.8 & & & 505.8 & 529.6 & 542.4 & 552.3 & 553.7 & 555.7 & & 572.7 & 582.9 & 515.8 & \\
\hline (PVt-PVt-1)/GDPt-1 (in percent) & $\ldots$ & & . & & & 4.7 & 3.6 & 1.8 & 1.3 & 0.2 & 0.2 & 2.0 & 0.3 & -0.3 & -1.1 & -0.1 \\
\hline
\end{tabular}

Sources: Grenada authorities; and IMF staff estimates and projections.

$1 /$ The coverage of the public sector is public and publicly guaranteed debt, including debt of public enterprises and the debt of the central government to the public sector.

$3 /$ Derived as $[\mathrm{i}-\mathrm{g}-\mathrm{r}(1+\mathrm{g}) /(1+\mathrm{g}+\mathrm{r}+\mathrm{gr})$ times previous period debt ratio, with $\mathrm{i}=$ nominal interest rate; $\mathrm{g}=$ real GDP growth rate, and $\mathrm{r}=$ growth rate of GDP deflator in U.S. dollar terms.

4/ Includes exceptional financing (i.e., changes in arrears and debt relief); changes in gross foreign assets; and valuation adjustments. For projections also includes contribution from price and exchange rate changes. $5 /$ Assumes that PV of private sector debt is equivalent to its face value.

7/ Defined as grants, concessional loans, and debt relief.

B/ Grant-equivalent financing includes grants provided directly to the government and through new borrowing (difference between the face value and the PV of new debt). 
Table A2b. Grenada: Sensitivity Analysis for Key Indicators of Public and Publicly Guaranteed External Debt, 2009-29 (In percent)

\begin{tabular}{|c|c|c|c|c|c|c|c|c|c|}
\hline & \multicolumn{9}{|c|}{ Projections } \\
\hline & 2009 & 2010 & 2011 & 2012 & 2013 & 2014 & 2019 & 2025 & 2029 \\
\hline \multicolumn{10}{|c|}{ PV of debt-to GDP ratio } \\
\hline Baseline & 75.6 & 76.3 & 73.8 & 70.9 & 67.1 & 63.5 & 48.7 & 34.8 & 24.3 \\
\hline \multicolumn{10}{|l|}{ A. Alternative Scenarios } \\
\hline A1. Key variables at their historical averages in $2009-20291 /$ & 75.6 & 70.1 & 65.7 & 62.2 & 59.1 & 57.4 & 35.2 & 22.4 & 22.6 \\
\hline A2. New public sector loans on less favorable terms in 2009-29 2/ & 75.6 & 77.3 & 75.8 & 73.6 & 70.3 & 67.1 & 54.7 & 45.2 & 37.8 \\
\hline \multicolumn{10}{|l|}{ B. Bound Tests } \\
\hline B1. Real GDP growth at historical average minus one standard deviation in 2010-11 & 75.6 & 79.1 & 81.0 & 77.8 & 73.6 & 69.7 & 53.4 & 38.2 & 26.7 \\
\hline B2. Export value growth at historical average minus one standard deviation in 2010-11 3/ & 75.6 & 82.0 & 90.3 & 87.3 & 83.4 & 79.0 & $\mathbf{5 5 . 0}$ & 36.1 & 24.8 \\
\hline B3. US dollar GDP deflator at historical average minus one standard deviation in 2010-11 & 75.6 & 77.6 & 76.5 & 73.5 & 69.5 & 65.8 & 50.4 & 36.0 & 25.2 \\
\hline B4. Net non-debt creating flows at historical average minus one standard deviation in $2010-114$ / & 75.6 & 83.7 & 90.4 & 87.4 & 83.5 & 78.9 & 54.8 & 36.1 & 24.8 \\
\hline B5. Combination of B1-B4 using one-half standard deviation shocks & 75.6 & 82.0 & 93.7 & 90.6 & 86.4 & 82.0 & 57.5 & 38.0 & 26.1 \\
\hline B6. One-time 30 percent nominal depreciation relative to the baseline in 20105 / & 75.6 & 107.7 & 104.3 & 100.2 & 94.8 & 89.7 & 68.8 & 49.1 & 34.3 \\
\hline
\end{tabular}

\section{PV of Debt-to-Exports ratio}

\section{Baseline}

A. Alternative Scenarios

A1. Key variables at their historical averages in 2009-2029 1/

A2. New public sector loans on less favorable terms in 2009-29 2

\section{B. Bound Tests}

B1. Real GDP growth at historical average minus one standard deviation in 2010-11

B2. Export value growth at historical average minus one standard deviation in 2010-11 3/

B3. US dollar GDP deflator at historical average minus one standard deviation in 2010-11

B4. Net non-debt creating flows at historical average minus one standard deviation in 2010-11 4/

B5. Combination of B1-B4 using one-half standard deviation shocks

B6. One-time 30 percent nominal depreciation relative to the baseline in 2010 5/

$\begin{array}{rrrrrrrrr}315.7 & 304.7 & 283.1 & 259.2 & 230.0 & 203.0 & \mathbf{1 6 1 . 2} & 112.2 & 77.4 \\ & & & & & & & & \\ 315.7 & 280.1 & 252.1 & 227.2 & 202.6 & 183.6 & \mathbf{1 1 6 . 3} & 72.3 & 72.0 \\ 315.7 & 309.0 & 290.6 & 268.8 & 241.0 & 214.7 & \mathbf{1 8 0 . 9} & 145.7 & 120.3 \\ & & & & & & & & \\ & & & & & & & & \\ 315.7 & 304.7 & 283.1 & 259.2 & 230.0 & 203.0 & \mathbf{1 6 1 . 2} & 112.2 & 77.4 \\ 315.7 & 417.8 & 572.9 & 527.8 & 472.7 & 418.1 & \mathbf{3 0 0 . 8} & 192.7 & 130.6 \\ 315.7 & 304.7 & 283.1 & 259.2 & 230.0 & 203.0 & \mathbf{1 6 1 . 2} & 112.2 & 77.4 \\ 315.7 & 334.2 & 346.7 & 319.4 & 286.1 & 252.5 & \mathbf{1 8 1 . 4} & 116.4 & 78.9 \\ 315.7 & 373.6 & 465.7 & 428.8 & 383.8 & 339.8 & \mathbf{2 4 6 . 4} & 158.7 & 107.7 \\ 315.7 & 304.7 & 283.1 & 259.2 & 230.0 & 203.0 & \mathbf{1 6 1 . 2} & 112.2 & 77.4\end{array}$

\section{Debt service-to-exports ratio}

\section{Baseline}

\begin{tabular}{rrrrrrrrr}
15.7 & 14.8 & 17.1 & 22.0 & 17.9 & 17.6 & $\mathbf{2 1 . 3}$ & 31.0 & 21.7 \\
& & & & & & & & \\
15.7 & 14.5 & 15.8 & 20.3 & 16.2 & 14.5 & $\mathbf{1 6 . 3}$ & 22.6 & 16.5 \\
15.7 & 14.8 & 16.5 & 21.2 & 16.8 & 17.0 & $\mathbf{1 9 . 4}$ & 44.1 & 36.1 \\
& & & & & & & & \\
& & & & & & & & \\
15.7 & 14.8 & 17.1 & 22.0 & 17.9 & 17.6 & $\mathbf{2 1 . 3}$ & 31.0 & 21.7 \\
15.7 & 18.9 & 30.2 & 41.7 & 34.6 & 37.0 & $\mathbf{4 7 . 3}$ & 53.5 & 36.8 \\
15.7 & 14.8 & 17.1 & 22.0 & 17.9 & 17.6 & $\mathbf{2 1 . 3}$ & 31.0 & 21.7 \\
15.7 & 14.8 & 18.6 & 25.2 & 20.9 & 23.0 & $\mathbf{2 8 . 6}$ & 32.4 & 22.2 \\
15.7 & 17.2 & 24.6 & 34.1 & 28.2 & 29.6 & $\mathbf{3 8 . 2}$ & 44.1 & 30.3 \\
15.7 & 14.8 & 17.1 & 22.0 & 17.9 & 17.6 & $\mathbf{2 1 . 3}$ & 31.0 & 21.7 \\
& & & & & & & & \\
-5.1 & -5.1 & -5.1 & -5.1 & -5.1 & -5.1 & $\mathbf{- 5 . 1}$ & -5.1 & -5.1 \\
\hline
\end{tabular}

A. Alternative Scenarios

A1. Key variables at their historical averages in 2009-29 1/

A2. New public sector loans on less favorable terms in 2009-29 2

\section{B. Bound Tests}

B1. Real GDP growth at historical average minus one standard deviation in 2010-11

B2. Export value growth at historical average minus one standard deviation in 2010-11 3/

B3. US dollar GDP deflator at historical average minus one standard deviation in 2010-11

B4. Net non-debt creating flows at historical average minus one standard deviation in 2010-114/

B5. Combination of B1-B4 using one-half standard deviation shocks

B6. One-time 30 percent nominal depreciation relative to the baseline in 2010 /

Grant element assumed on residual financing (i.e., financing required above baseline) $6 /$
Source: Grenada authorities; and IMF staff projections and simulations.

1/ Variables include real GDP growth, growth of GDP deflator (in U.S. dollar terms), non-interest current account in percent of GDP, and non-debt creating flows

2/ Assumes that the interest rate on new borrowing is by 2 percentage points higher than in the baseline, while grace and maturity periods are the same as in the baseline.

3/ Exports values are assumed to remain permanently at the lower level, but the current account as a share of GDP is assumed to return to its baseline level after the shock (implicitly assuming an offsetting adjustment in import levels).

$4 /$ Includes official and private transfers and FDI.

$5 /$ Depreciation is defined as percentage decline in dollar/local currency rate, such that it never exceeds 100 percent.

6/ Applies to all stress scenarios except for A2 (less favorable financing) in which the terms on all new financing are as specified in footnote 2. 
Figure 1. Grenada: Indicators of Public Debt Under Alternative Scenarios, 2009-29 1/

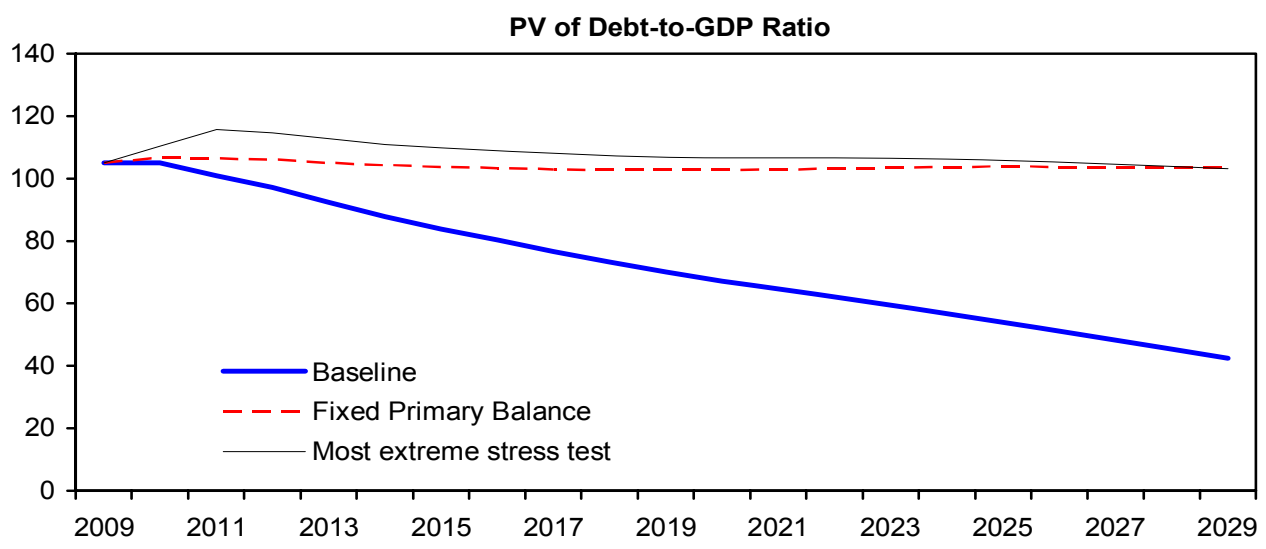

PV of Debt-to-Revenue Ratio 2I

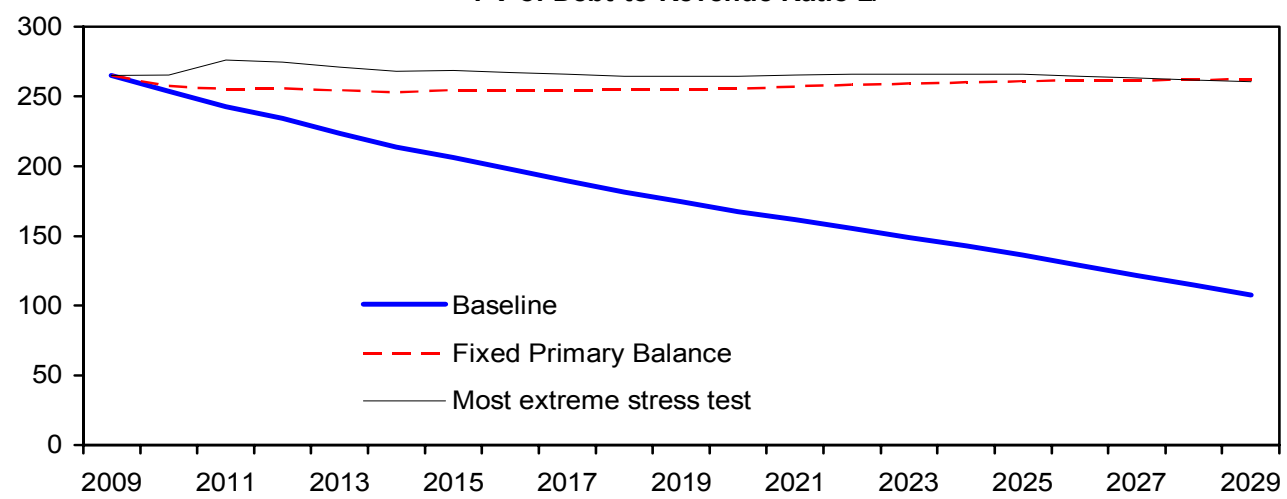

Debt Service-to-Revenue Ratio 2/

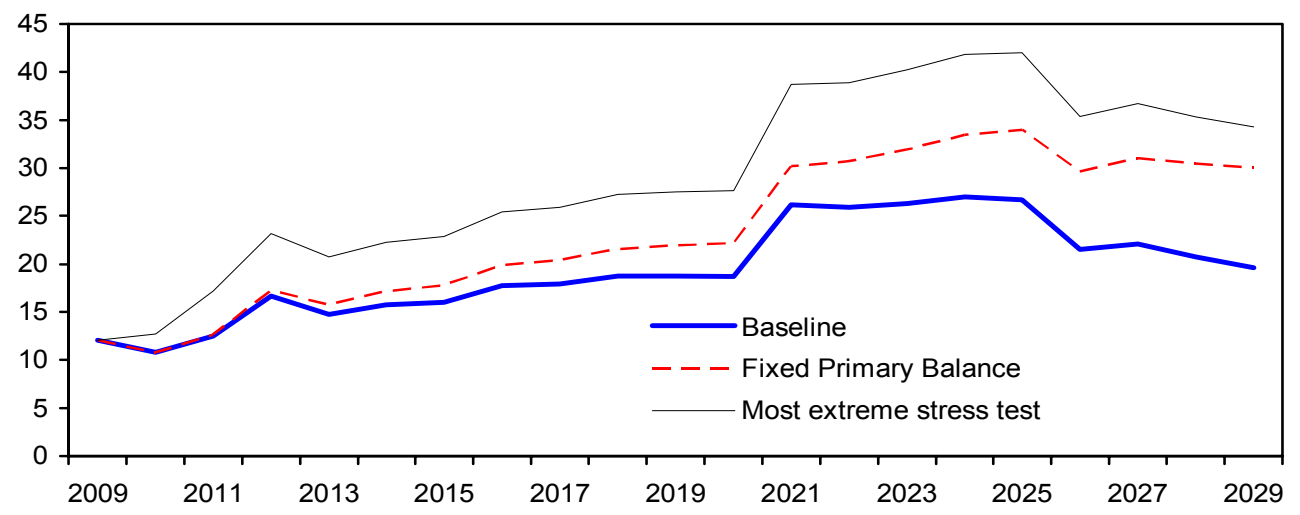

Sources: Grenada authorities; and IMF staff estimates and projections.

$1 /$ The most extreme stress test is the test that yields the highest ratio in 2019. It corresponds to real GDP growth at historical average minus one standard deviations in 2010-11 in panels one and two and to a one-time real depreciation of 30 percent in 2010 in panel three.

2/ Revenues are defined inclusive of grants. 
Figure 1a. Grenada: Indicators of Public Debt Under Alternative Scenarios, 2009-29 1/

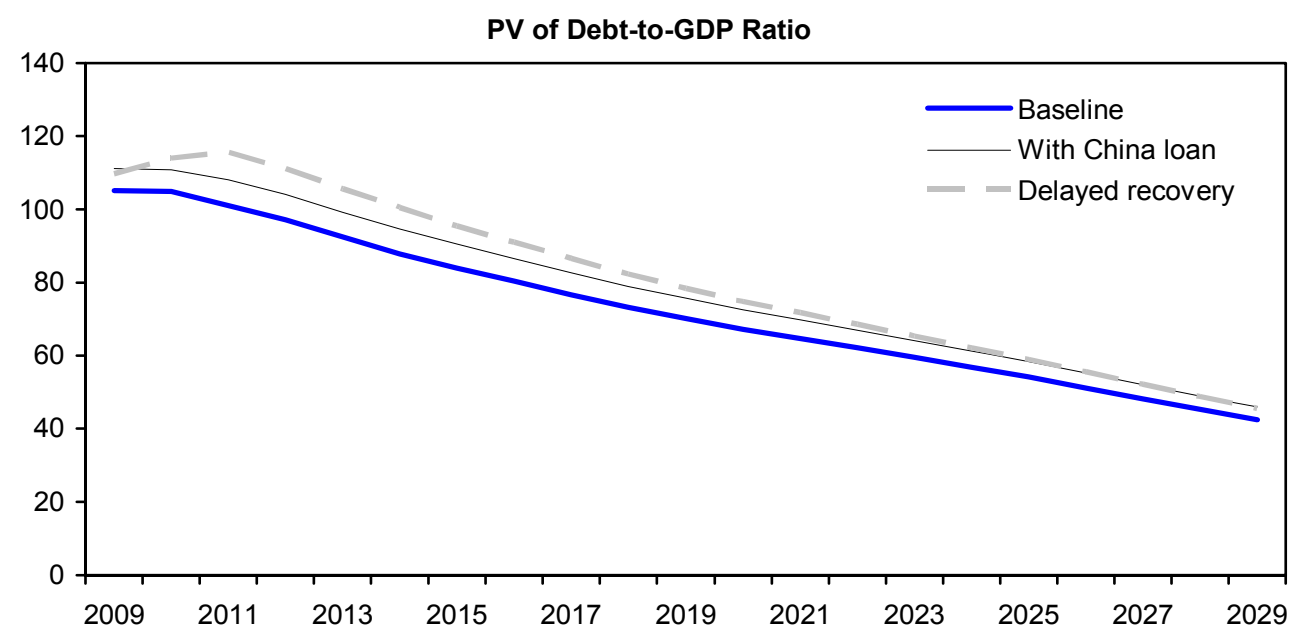

PV of Debt-to-Revenue Ratio 1/
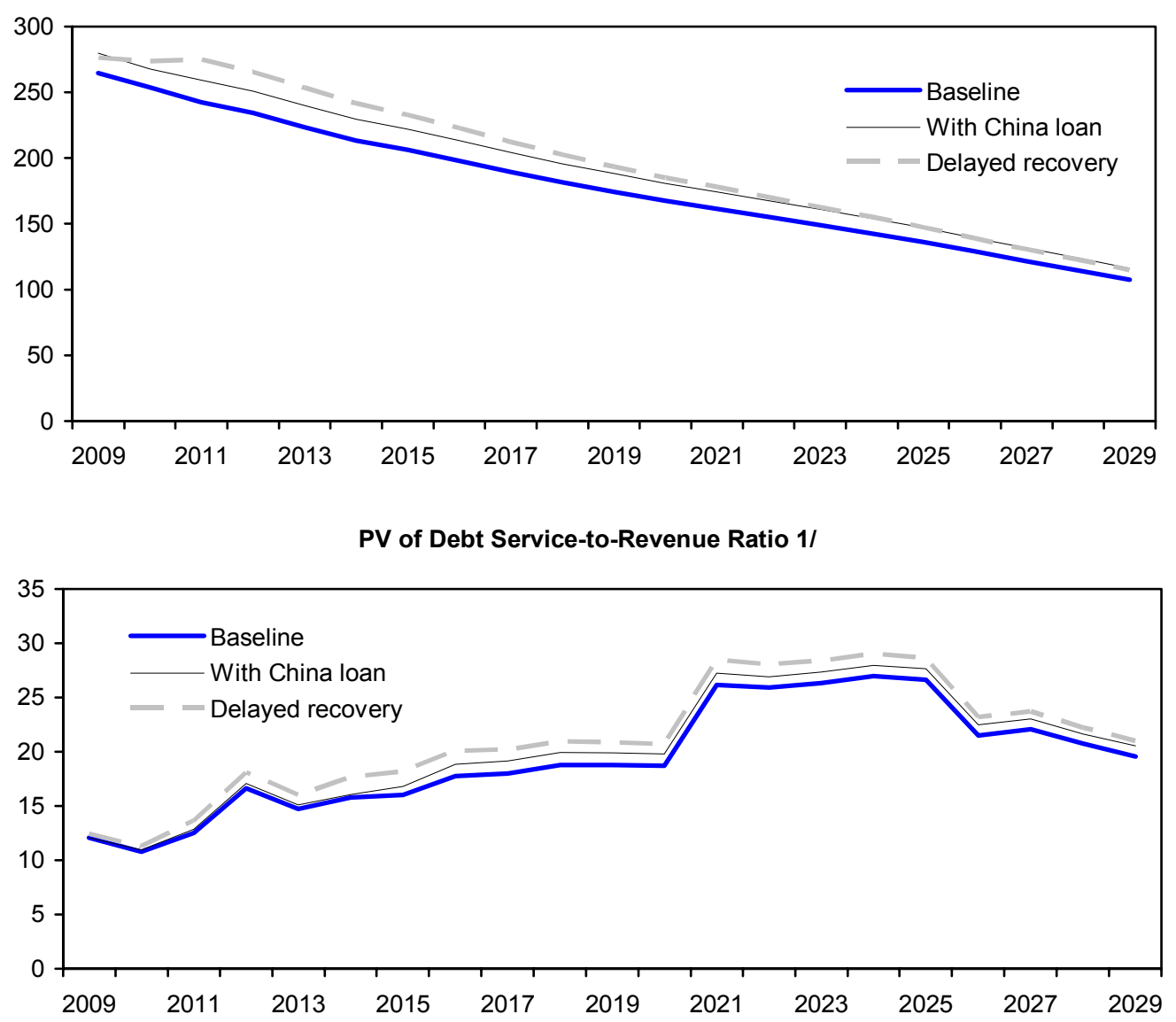

Sources: Grenada authorities; and IMF staff estimates and projections.

1/ Revenues are defined inclusive of grants. 
Figure 2. Grenada: Indicators of Public and Publicly Guaranteed External Debt under Alternatives Scenarios, 2009-29 1/

a. Debt Accumulation

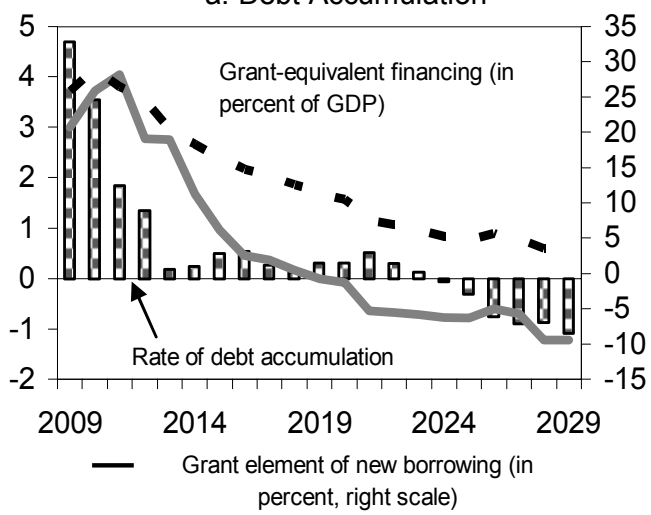

c. PV of Debt-to-Exports ratio

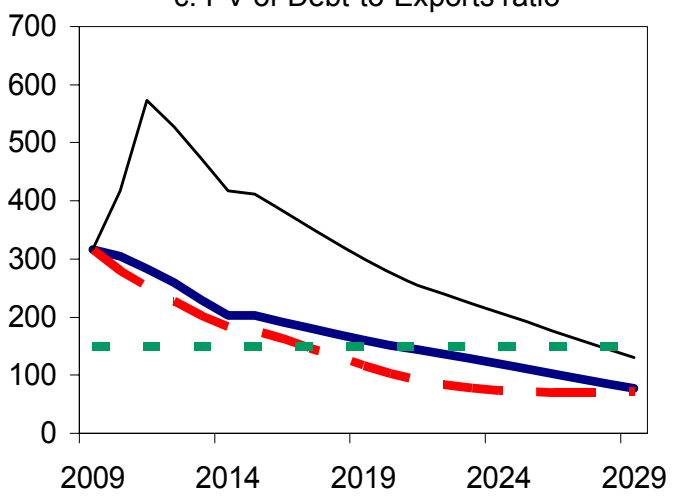

e. Debt service-to-exports ratio

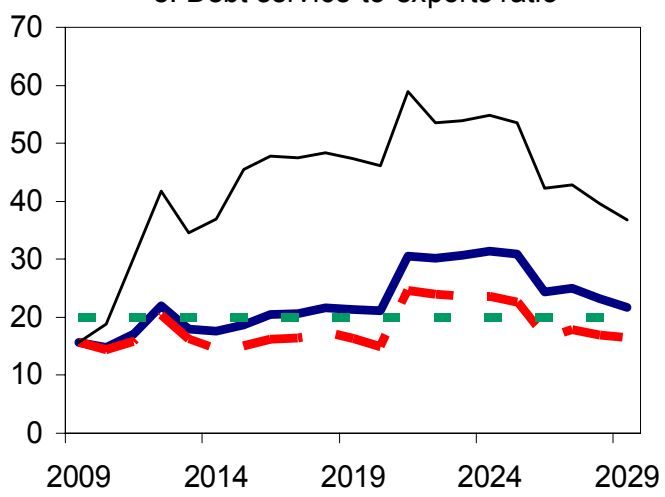

b. PV of debt-to GDP ratio

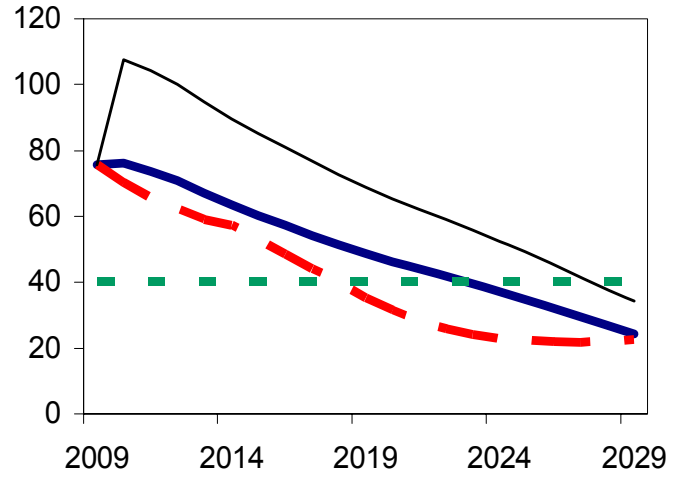

d. PV of debt-to-revenue ratio

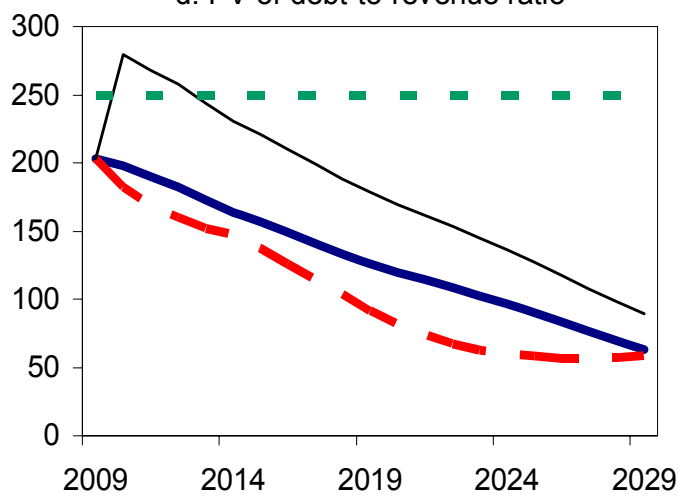

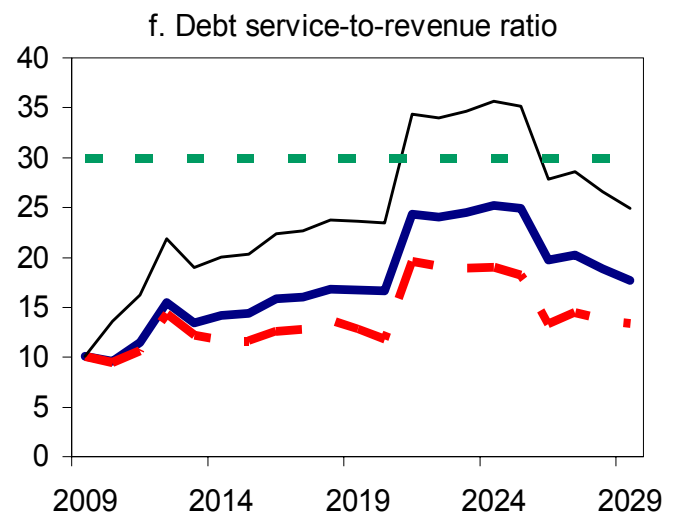

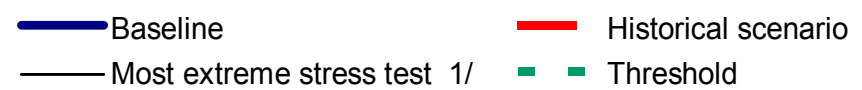

Source: IMF staff projections, and simulations.

1/ The most extreme stress test is the test that yields the highest ratio in 2019. In figure b. it corresponds to a one-time depreciation shock; in c. to an exports shock; in d. to a one-time depreciation shock; in e. to an exports shock and in picture f. to a one-time depreciation shock. 
Figure 2a. Grenada: Indicators of Public and Publicly Guaranteed External Debt under Alternatives Scenarios, 2009-29 1/

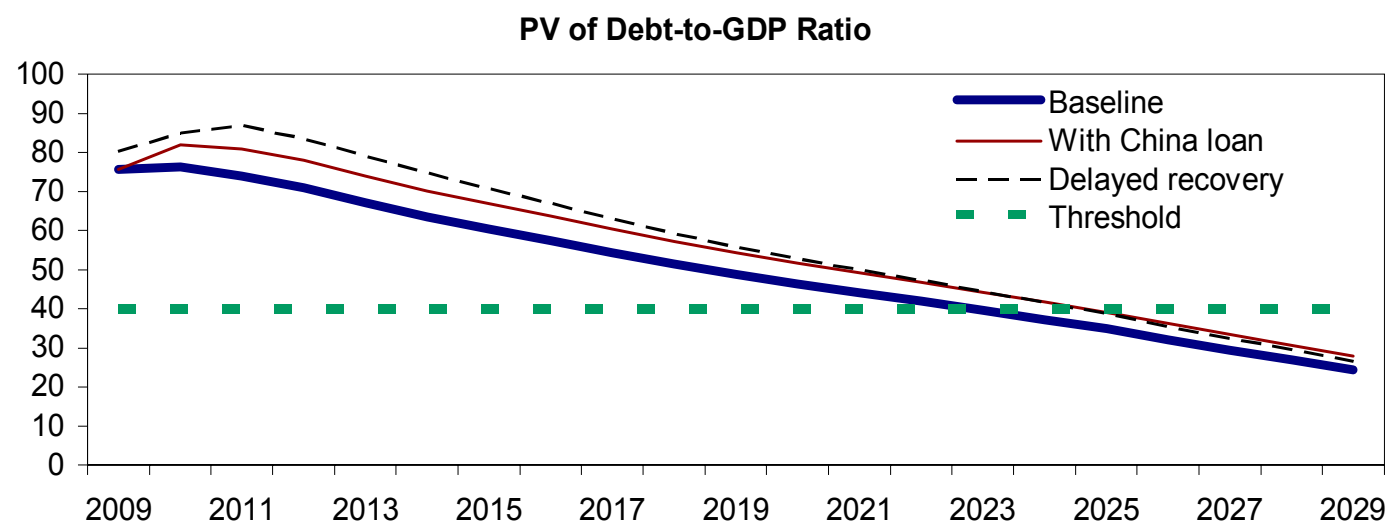

PV of Debt-to-Exports Ratio

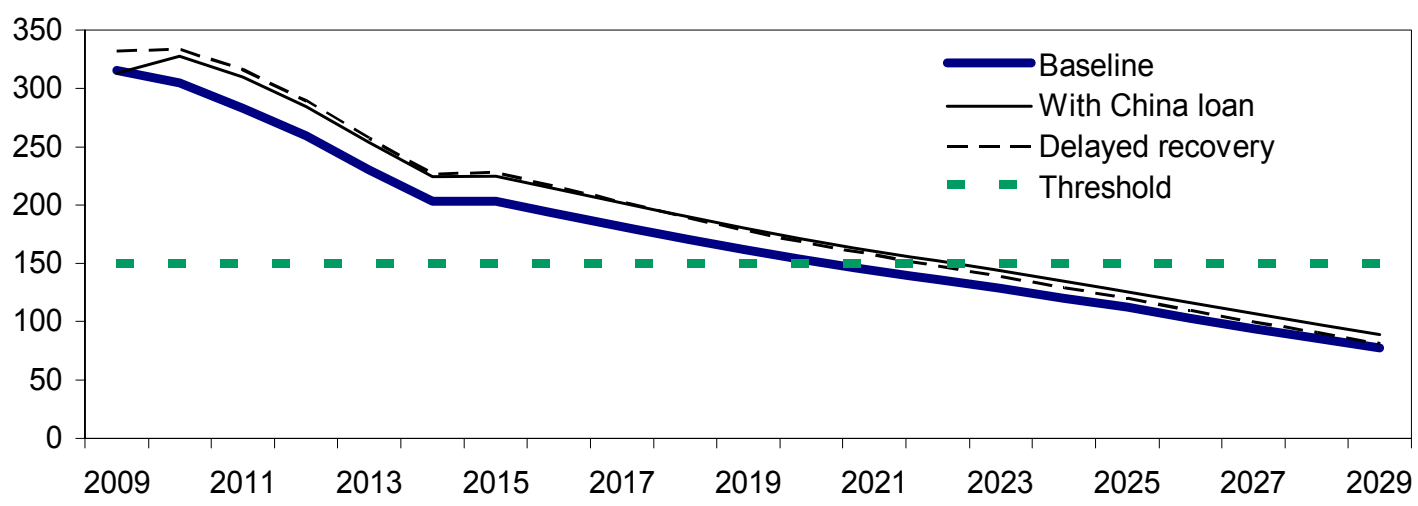

PV of Debt Service-to-Exports Ratio

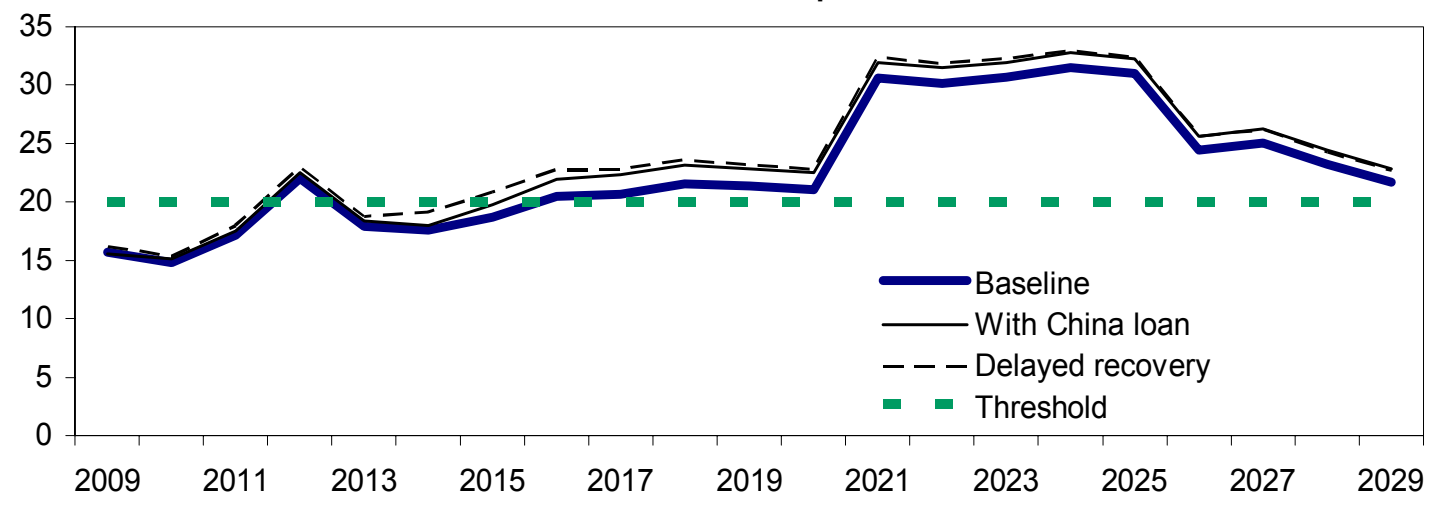

Source: IMF staff projections, and simulations. 


\section{INTERNATIONAL MONETARY FUND}

GRENADA

Third Review Under the Three-Year Arrangement Under the Poverty Reduction and Growth Facility, Requests for Modification of Quantitative Performance

Criterion and Augmentation, and Financing Assurances Review

\section{Informational Annex}

Prepared by Western Hemisphere Department

May 20, 2009

Contents

Page

Appendices

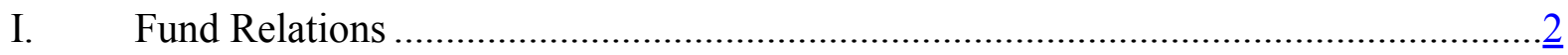

II. Relations with the World Bank Group ...................................................................

III. Relations with the Caribbean Development Bank ……............................................ 9

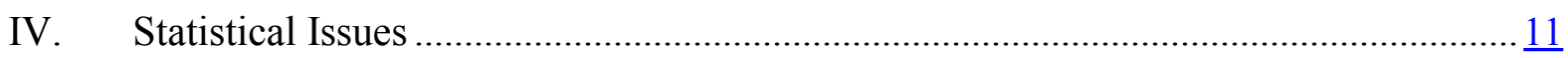




\section{Appendix I. Grenada: Fund Relations}

(As of April 30, 2009)

I. Membership Status: Joined: August 27, 1975.

II. General Resources Account:

SDR Million

Quota

Fund Holdings of Currency

Reserve Position

III. SDR Department:

Net cumulative allocation

Holdings
11.70

12.80

0.00

SDR Million

0.93

0.46

SDR Million

1.10

6.95
Percent of Quota

100.00

109.39

0.00

Percent of

Allocation

100.00

49.83

Percent of

Quota

9.38

59.40

V. Financial Arrangements:

$\begin{array}{ccccc}\text { Type } & \begin{array}{c}\text { Date of } \\ \text { Arrangement }\end{array} & \begin{array}{c}\text { Expiration } \\ \text { Date }\end{array} & \begin{array}{c}\text { Amount Approved } \\ \text { (SDR Million) }\end{array} & \begin{array}{c}\text { Amount Drawn } \\ \text { (SDR Million) }\end{array} \\ \text { PRGF } & \begin{array}{l}\text { Apr. 17, 2006 } \\ \text { Apr } .16,2010\end{array} & 11.99 & 6.95\end{array}$

VI. Projected Obligations to Fund

(SDR Million; based on existing use of resources and present holdings of SDRs)

Forthcoming

$\begin{array}{llllll}\text { Principal } & 1.10 & 0.00 & 0.16 & 0.31 & 0.31 \\ \text { Charges/Interest } & \underline{0.04} & \underline{0.04} & \underline{0.04} & \underline{0.03} & \underline{0.03} \\ \text { Total } & 1.14 & 0.04 & 0.19 & 0.35 & 0.35\end{array}$

VII. Implementation of HIPC and MDRI Initiatives: Not Applicable 
VIII. Safeguards Assessment: Under the Fund's safeguards assessment policy, the Eastern Caribbean Central Bank (ECCB) is subject to a full safeguards assessment under a four-year cycle. The most recent assessment was completed in July 2007, and concluded that the ECCB continues to have appropriate control mechanisms in place, which have strengthened since the first safeguards assessment completed in 2003. ECCB management places emphasis on good governance and sound controls, and has enhanced the bank's transparency and accountability since the last assessment, including the publications of financial statements that comply with International Financial Reporting Standards. The assessment made some recommendations to sustain the ECCB's safeguards framework going forward.

IX. Exchange Arrangement: Grenada is a member for the ECCB, which manages monetary policy and the exchange system for its eight members. The common currency, the Eastern Caribbean dollar, has been pegged to the U.S. dollar at the rate of EC $\$ 2.70$ per U.S. dollar since July 1976. In practice, the ECCB has operated like a quasi-currency board, maintaining foreign exchange backing of its currency and demand liabilities of close to 100 percent. Grenada accepted the obligations of Article VIII, Sections 2, 3, and 4 in January 1994. It maintains an exchange system free of restrictions on the making of payments and transfers for current international transactions.

X. Article IV Consultation: Grenada is on a 24-month consultation cycle. The last Article IV consultation was concluded by the Executive Board on September 26, 2007 (IMF Country Report No. 08/351).

XI. FSAP Participation: Grenada participated in the regional Eastern Caribbean Currency Union FSAP conducted in September and October 2003. The Financial System Stability Assessment is IMF Country Report No. 04/293.

\section{Technical Assistance:}

\section{Caribbean Regional Technical Assistance Centre (CARTAC)}

CARTAC has provided wide-ranging assistance in developing a Medium-Term Macroeconomic Framework (as part of the Structural Adjustment Technical Assistance Program); preparing to implement a VAT, building upon previous work, including draft VAT/excise laws (prepared by LEG) and a VAT sensitivity study and training/publicity tasks (undertaken with CARTAC/FAD assistance); assisting customs with ASYCUDA/ ASYCUDA++ and with the exchange of information with inland revenue; drafting legislation to establish the single supervisory agency, Grenada Authority for the Regulation of the Financial Institutions (GARFIN) and supporting the newly established agency; and training for nonbank supervisors. CARTAC has also provided substantial assistance in improving the production and dissemination of macroeconomic statistics, including national accounts compilation; rebasing of the consumer price index and GDP constant prices series; 
initiating work to prepare export-import price indices; training in the processing of trade data; and improving external sector statistics as part of a major CARTAC/ECCB project.

Other Technical Assistance (2007-09)

FAD and LEG have provided extensive assistance in tax policy and administration. In particular, FAD and LEG have assisted in the design and drafting of a VAT and related changes to excise taxes. LEG has also assisted with training for tax officials and with the finalization of the VAT and excise laws as well as with drafting of an investment promotion bill. A series of FAD missions have provided further assistance on implementing a VAT and on tax policy, tax and customs administration and reforms as well as on income tax reform and tax incentives system. 


\section{Appendix II. Grenada - Relations with the World Bank Group}

(As of April 24, 2009)

In September 2005, the Eastern Caribbean Sub-Region Country Assistance Strategy (CAS) for FY06-09 was presented to the Board of the World Bank. The strategy supports the sub-region's development agenda through two main pillars: (1) stimulating growth and improving competitiveness; and (2) reducing vulnerability, by promoting greater social inclusion and strengthening disaster risk management. Recognizing the OECS countries' weakened creditworthiness due to high debt ratios, Bank activities will focus on leveraging available donor grant financing. Following the recommendations of the recently completed growth and competitiveness study for the OECS, IBRD and IDA support would focus on providing technical and financial assistance for interventions to support the two main pillars. An indicative Base Case lending scenario consisted of about US\$51.3 million in IDA resources for the four OECS IDA eligible countries. An OECS CAS progress report was presented to the Board in June 2008.

\section{A. Projects}

There are nine active World Bank projects in Grenada for a net commitment of approximately US $\$ 48.5$ million of which US\$22.6 million has been disbursed.

The HIV/AIDS Prevention and Control Program, approved in July 2002 for US\$6.0 million, is funded under the multi-country Adaptable Program Loan (APL) for the Caribbean region. Its objectives are: (i) to curb the spread of the HIV/AIDS pandemic; (ii) to reduce the morbidity and mortality attributed to HIV/AIDS; (iii) to improve the quality of life for persons living with HIV/AIDS; and (iv) to develop a sustainable organizational and institutional framework for managing the HIV/AIDS epidemic. This project is scheduled to close by June 2009 .

The Grenada (OECS) Education Development Project, approved in June 2003 for US\$8.0 million as part of a multi-country APL, is a follow-up to an earlier education project. Its objective is to build human capital, with a view to contributing to economic diversification and more sustainable growth. Key objectives are: (i) to increase equitable access to secondary education; (ii) to improve the quality of the teaching and learning processes, with more direct interventions at the school level and an increased focus on student-centered learning, and (iii) to strengthen management of the education sector and improve governance of schools. The World Bank has approved additional financing in the amount of US\$1.9 million and the project should be effective by late May 2009. The additional resources will finance the costs associated with scaling up those components curtailed when the original project was restructured after Hurricane Ivan in November 2004, namely improving education quality and strengthening management. 
The Hurricane Ivan Emergency Project was approved in November 2004 for US\$10 million, with an additional US\$9.8 million being added in September 2005. This emergency assistance for Grenada was implemented to respond to the effects of a devastating hurricane that hit the island in September 2004. The project supports Grenada's recovery efforts through the financing of critical imports and rehabilitation activities in key social sectors.

The Telecommunications and ICT Development Project, approved in May 2005 in the amount of US $\$ 540,000$, aims at improving the access, quality, and use of telecommunications and ICT services to achieve socio-economic development in the Organization of Eastern Caribbean States (OECS). The project has the following four components: Component 1 -strengthen the national and regional regulatory frameworks and promote additional competition in the telecommunications sector; Component 2-review current universal access policy, create related guidelines, and provide financial support to establish a Universal Service Fund (USF); Component 3-improve growth and competitiveness in ICT-enabled services through utilization of broadband infrastructure; and Component 4 - ensure management and administration of the overall project.

\section{The Public Sector Modernization Technical Assistance Project was approved in} December 2005 to support the modernization of Grenada's public sector. The US\$3.5 million IDA Credit will finance a project that has the following components: Component 1 - (i) the strategic review of the proposed organizations and functions for conversion to Executive Agencies status; (ii) the preparation of detailed, modernization and financing plans for each conversion; (iii) the preparation of a Policy Framework for Executive Agencies; and (iv) the preparation of enabling legislation-including the preparation of a draft Executive Agencies bill to be presented to Cabinet and Parliament under Grenada's legal framework; Component 2-support for the strengthening of the Small Business Development Centre (SBDC) of the Grenada Industrial Development Corporation (GIDC) that will provide technical assistance and training to the micro/small segment of the business community; Component 3-support for Grenada to take the lead to jointly procure select goods and services with other OECS countries; and Component 4-strengthen the Public Sector Reform Unit by providing financial and technical resources and training on key policy areas.

The Caribbean Catastrophe Risk Insurance Facility (CCRIF) was approved in March 2007 as the world's first ever multi-country catastrophe insurance pool. The US $\$ 4.5$ million IDA Credit will finance Grenada's contribution to the insurance pool over three years. The CCRIF has enabled governments to purchase catastrophe insurance coverage against adverse natural events, such as a major earthquake or hurricane. The CCRIF allows participating countries to pool their country-specific risks into one, better-diversified portfolio, resulting in a substantial reduction in the premium cost of $45-50$ percent.

The Grenada Technical Assistance Project was approved on March 13, 2008 as a US\$2.8 million IDA Credit. The Project's developmental objectives are: (i) to improve the efficiency and effectiveness of Customs, (ii) to improve the efficiency of tax administration and decrease transaction costs of paying taxes and consequently increase tax compliance, (iii) to modernize investment promotion, and, (iv) to enhance the Government's support to 
the export sector through improved access to trade information and the strengthening the capacity of the Bureau of Standards to provide conformity assessment and quality assurance. The medium-to-long-term direct impact of the project is likely to be substantial, including: better quality service and reduced clearance time at customs; reduced time and lower transaction cost for paying taxes and hence improved compliance; greater access to trade data and quality assurance support for exporters; and increased investments as a result of a more streamlined and faster system for investment approval in Grenada.

The OECS E-Government for Regional Integration Program was approved by the Board on May 27, 2008. This project consists of a US\$2.4 million IDA Credit to Grenada and is designed to promote the efficiency, quality, and transparency of public services through the delivery of regionally integrated e-government applications that take advantage of economies of scale. The program is structured in phases. Phase 1 focuses on cross-sectoral e-government issues, as well as on specific applications in the public finance area (including Public Financial Management or PFM, tax, customs and procurement), and also includes an e-government in health pilot project (possibly together with preparatory and complementary activities in other social and productive sectors). Subsequent phases of the program are expected to deepen the assistance provided under Phase 1, while expanding the program to cover other sectors, in particular, education, agriculture, tourism, postal, among others that may emerge during the early stages of implementation of Phase 1.

OECS (Grenada) Skills for Inclusive Growth, was approved on January 15, 2009 for US\$3 million IDA Credit which will fund the second phase of the multi-country APL and will support Grenada's efforts to increase the employability of youth through public/private sector partnerships for technical and life skills training that is demand driven. This objective has three intermediate outcomes with associated lines of action: (a) to increase job-related competencies among unemployed youth through the establishment of a competitive training mechanism that supports the financing and delivery of demand driven training; (b) to improve the quality and value of training in Grenada and enhance OECS collaboration in training through the adoption of an occupational standards framework that is validated locally and recognized regionally; and (c) to strengthen institutional capacity to plan, implement, and monitor training.

\section{B. Economic and Sector Work}

The Bank has completed a series of analytical studies relating to public sector capacity in the OECS including a number of Public Expenditure Reviews, an Institutional and Organizational Capacity Review and, in late 2007, a Country Fiduciary Assessment. The Bank also prepared an OECS study on Growth and Competitiveness (2005), an OECS Skills Enhancement Policy Note (2006), a Caribbean Air Transport Report (2006), and a regional study on Crime, Violence, and Development: Trends, Costs, and Policy Options in the Caribbean (2007). In addition, an OECS Private Sector Financing Study and the OECS Tourism Backward Linkages Study were completed in 2008. The publication 
"Caribbean-Accelerating Trade Integration: Policy Options for Sustained Growth, Job Creation and Poverty Reduction" was released in the Fall of 2008.

Grenada will also benefit from ongoing and planned analytical and advisory activities including the following: a CARICOM study on Managing Nurse Migration and a preparatory study aimed at developing a Caribbean-wide Regional Energy Strategy.

\section{Financial Relations}

(In millions of U.S. dollars)

\begin{tabular}{|l|c|c|c|}
\hline Operation & $\begin{array}{c}\text { Original } \\
\text { Principal }\end{array}$ & Available $^{\mathbf{1}}$ & Disbursed $^{\mathbf{1}}$ \\
\hline $\begin{array}{l}\text { E-Government for Regional Integration } \\
\text { Program }\end{array}$ & 2.40 & 2.23 & 0.00 \\
\hline Grenada Technical Assistance Project & 1.86 & 1.78 & 0.00 \\
\hline The Caribbean Catastrophe Risk Insurance & 4.50 & 0.85 & 3.61 \\
\hline $\begin{array}{l}\text { The Public Sector Modernization Technical } \\
\text { Assistance Project }\end{array}$ & 3.50 & 2.95 & 0.76 \\
\hline $\begin{array}{l}\text { Telecommunications \& ICT Development } \\
\text { Project }\end{array}$ & 0.54 & 0.30 & 0.26 \\
\hline Hurricane Ivan Emergency Recovery Project & 19.77 & 5.58 & 9.84 \\
\hline Grenada Education Development Project & 9.90 & 3.10 & 5.89 \\
\hline HIV/AIDS Prevention And Control Program & 6.04 & 2.94 & 2.25 \\
\hline Total & $\mathbf{4 8 . 5 1}$ & $\mathbf{1 9 . 7 3}$ & $\mathbf{2 2 . 6 1}$ \\
\hline
\end{tabular}

1/ Amounts may not add up to Original Principal due to changes in the SDR/US exchange rate since signing.

Disbursements and Debt Service (Fiscal Year ending June 30)

\begin{tabular}{|l|c|c|c|c|c|c|c|c|c|c|}
\hline & $\mathbf{2 0 0 0}$ & $\mathbf{2 0 0 1}$ & $\mathbf{2 0 0 2}$ & $\mathbf{2 0 0 3}$ & $\mathbf{2 0 0 4}$ & $\mathbf{2 0 0 5}$ & $\mathbf{2 0 0 6}$ & $\mathbf{2 0 0 7}$ & $\mathbf{2 0 0 8}$ & $\mathbf{2 0 0 9} *$ \\
\hline Total disbursements & 1.99 & 2.46 & 2.78 & 6.30 & 2.77 & 5.93 & 4.27 & 5.83 & 7.48 & 1.23 \\
\hline Repayments & 0.07 & 0.06 & 0.06 & 0.07 & 0.22 & 0.63 & 1.10 & 1.49 & 1.62 & 1.49 \\
\hline Net disbursements & 1.92 & 2.40 & 2.71 & 6.23 & 2.55 & 5.30 & 3.17 & 4.35 & 5.85 & -0.26 \\
\hline Interest and fees & 0.08 & 0.22 & 0.29 & 0.39 & 0.48 & 0.53 & 0.65 & 0.78 & 0.82 & 0.66 \\
\hline
\end{tabular}

* July 2008 - March 2009 


\section{Appendix III. Grenada-Relations with the Caribbean Development Bank}

(As of April 24, 2009)

\section{A. New Projects and Technical Assistance in 2008}

Grenada, since Hurricane Ivan in 2004, continues to benefit from special financing from the Caribbean Development Bank (CDB), designed to yield a concessional grant element of 35 percent. These loans offer longer maturities and grace periods, as well as lower interest rates than those applied in the Bank's ordinary operations.

\section{Rockfall and Landslip (Additional Loan and Variation in Scope)}

In May 2008, a loan of US $\$ 3.7$ million was approved to finance cost overruns (on the original Rockfall and Landslip project that was approved in 2005), as well as additional works to mitigate rockfall hazards at selected sites and to restore damaged retaining structures at selected locations along the main road network. The original loan of US\$5.7 million was approved for similar mitigation works.

\section{Institutional Strengthening-The Ministry of Communications, Works and Transport}

In March 2008, a technical assistance grant of US\$466,200 was approved to assist with the establishment of a Project Implementation and Management Unit within the Ministry, and with the conduct of an organizational assessment of the Ministry by consultants with a view to improving project implementation and management capacity.

\section{B. Ongoing Activities}

Loan disbursements continued for projects under implementation. These included capital works: (i) to upgrade and rehabilitate physical infrastructure including the road and bridge network as well as school infrastructure; (ii) to reduce the risk of rock fall and landslip events in the aftermath of natural hazards; (iii) to improve the shelter conditions of low-income households through the provision of 116 serviced lots; and (iv) to facilitate urban redevelopment. 


\section{Financial Relations}

(As at March 2009)

(In millions of U.S. dollars)

\begin{tabular}{|c|c|c|c|c|c|}
\hline Item & 2005 & 2006 & 2007 & 2008 & 2009 \\
\hline Cumulative total credit approved $^{\top}$ & 152.6 & 163.6 & 171.1 & 174.8 & 174.9 \\
\hline Cumulative disbursements $^{2}$ & 112.8 & 130.0 & 143.3 & 150.0 & 150.9 \\
\hline Outstanding debt (end of period) & 77.6 & 91.6 & 101.8 & 106.4 & 106.1 \\
\hline \multicolumn{6}{|l|}{ Disbursements } \\
\hline Ordinary Capital Resources & 5.6 & 3.3 & 1.0 & 0.5 & 0.02 \\
\hline Special Development Fund & 8.4 & 9.8 & 7.0 & 3.5 & 0.7 \\
\hline Other Special Fund Resources & 0.6 & 3.9 & 6.7 & 4.4 & 0.2 \\
\hline \multicolumn{6}{|l|}{ Amortization $^{3}$} \\
\hline Ordinary Capital Resources & 1.2 & 1.5 & 1.4 & 1.8 & 0.6 \\
\hline Special Development Fund & 1.6 & 1.6 & 1.0 & 0.7 & 0.2 \\
\hline Other Special Fund Resources & 0.2 & 0.3 & 0.2 & 0.2 & 0.04 \\
\hline \multicolumn{6}{|l|}{ Interest and Commitment Fees } \\
\hline Ordinary Capital Resources & 1.6 & 1.9 & 2.0 & 1.8 & 0.4 \\
\hline Special Development Fund & 0.7 & 0.9 & 0.3 & 1.1 & 0.3 \\
\hline Other Special Fund Resources & 0.2 & 0.2 & 1.1 & 0.4 & 0.1 \\
\hline
\end{tabular}

Source: Caribbean Development Bank.

${ }^{1}$ Loans to Government of Grenada (does not include grants).

${ }^{2}$ Including valuation adjustments.

${ }^{3}$ Ordinary capital resources (OCR) are loans on non-concessional terms. Special development funds (SDF) and other special fund (OSF) resources are soft loans. Commitment fees apply only to OCR. 


\section{APPENDIX IV. GRENADA-STATISTICAL ISSUES}

As of May 11, 2009

\section{Assessment of Data Adequacy for Surveillance}

General: Data provision has some shortcomings, but is broadly adequate for surveillance. Identified deficiencies need to be addressed in the context of a thorough implementation of the System of National Accounts 1993 and a revision of the national accounts' base year (currently dating from 1990). The IMF, through the Caribbean Regional Technical Assistance Center, has been providing specialized technical advice to Grenada on the critical area of export-import price indices compilation and dissemination.

National Accounts: National accounts are provided annually but are subsequent to frequent revisions - major revisions were undertaken in 2007 to extend coverage of national accounts to the offshore university, as well as to improve construction activity estimates. There are a number of deficiencies in the real sector statistics. GDP by expenditure is available only with long lags, and real GDP estimates for the tourism sector are not computed. The estimation of gross fixed capital formation and sectoral price deflators needs to be improved.

Price statistics: Consumer prices are the only real sector data provided in between IMF missions; however these data are subject to frequent revisions. The basket used to compute the consumer price index was last updated in 2000. A producer price index is not available.

Labor statistics: Labor statistics are limited and outdated, with 1998 being the most recent year for which data are available. There are no regular wage and unemployment data. Data collected during the 2001 population census are still unprocessed. The Central Statistical Office (CSO) is working with the International Labor Organization (ILO) to improve the coverage of labor market statistics and is also conducting a Country Poverty Assessment, with assistance of the Caribbean Development Bank.

Government finance statistics: The reporting of central government data has improved in recent years, with quarterly data being provided to the ECCB, WHD, and other users in Fund economic classification format with lags of about two months. However, there appear to be inaccuracies in the composition of public expenditure. Moreover, capital expenditures could include current expenditure items, and the nature of expenditures in the Public Sector Improvement Program needs to be scrutinized carefully. In addition, spending on outsourced activities is not broken down into the appropriate categories, but rather grouped into a single category.

The coverage of the rest of the public sector is very limited, and there are no consolidated public sector accounts. There is no systematic reporting of information to the ministry of finance. Annual statements for some public enterprises are provided during Fund missions. It would be useful to institute a mechanism under the public finance for the regular reporting of financial data pertaining to the rest of the public sector, in line with the new Public Finance Management Act of 2007 requiring public enterprises to submit audited financial statements within four months after the close of the financial year. 
Monetary statistics: Monetary data are compiled by the ECCB on a monthly basis and reported regularly to the Fund. There is a need to improve the institutional coverage that currently only comprises the ECCB and commercial banks. Full implementation of recommendations made by the 2003 and 2005 monetary and financial statistics missions should ensure consistency with international best practice.

While noting some recent improvements, the 2007 data ROSC mission identified the following main shortcomings in the ECCB's monetary statistics: (i) the methodological soundness of monetary statistics can be improved by adopting internationally accepted concepts and definitions, expanding institutional coverage, and revising the classifications of financial instruments and the basis for recording; (ii) transparency can be improved, for example, by releasing monetary data to all users at the same time and strengthening the validation of the disseminated data; (iii) the timeliness of the dissemination of data on broad money and credit aggregates can be improved to meet best international practices; and (iv) the access to officially disseminated data and metadata can be improved. Following the mission's recommendation, the ECCB revised its definition of broad money and the relevant changes have been reflected from the January 2009 monetary statistics.

Balance of payments: The ECCB compiles balance of payments statistics on an annual basis, using information collected by the CSO. The data are reported to the IMF for publication in the IFS and Balance of Payments Yearbook.

The statistics are based primarily on information collected from surveys of establishments; however, these surveys are not comprehensive and the response rates are usually poor. Merchandise trade statistics have traditionally been more reliable and are available by SITC classification on a quarterly basis. The reliability and comprehensiveness of the merchandise trade statistics have suffered considerably in the aftermath of Hurricane Ivan (September 2004) and reporting is mostly fully back on track.

Enhanced data sources and better compilation procedures are needed to improve the accuracy of the balance of payments statistics. Moreover, efforts should be undertaken to compile quarterly balance of payments statistics and the annual international investment position statement.

External and domestic debt statistics: The database for government external debt is quite comprehensive, and can be used to provide detailed and reasonably up-to-date breakdowns of disbursements and debt service. However, data availability on domestic debt, governmentguaranteed debt, and debt of public enterprises is limited, and there is no data on private external debt.

\section{Data Standards and Quality}

While Grenada has participated in the Fund's General Data Dissemination System since March 2001, most of the metadata have not been updated since late 2002 .

The 2007 regional data ROSC on monetary statistics provides an assessment of the ECCB's monetary statistics.

\section{Reporting to STA}

The International Financial Statistics page includes data on exchange rates, international liquidity, monetary statistics, prices, balance of payments, national accounts, and population. 
The authorities do not report fiscal data for publication in IFS.

The ECCB provides data to the IMF for publication in the Balance of Payments Yearbook.

Grenada has not provided any fiscal data, either on a GFSM 2001 basis, or a cash basis, for presentation in the GFS Yearbook. The ECCB disseminates Grenada's quarterly GFS data in its Economic and Financial Review. 


\section{Grenada: Table of Common Indicators Required for Surveillance}

As of May 18, 2009

\begin{tabular}{|c|c|c|c|c|c|}
\hline & $\begin{array}{c}\text { Date of } \\
\text { latest } \\
\text { observation }\end{array}$ & $\begin{array}{l}\text { Date } \\
\text { received }\end{array}$ & $\begin{array}{c}\text { Frequency } \\
\text { of } \\
\text { Data }^{1}\end{array}$ & $\begin{array}{c}\text { Frequency of } \\
\text { Reporting }\end{array}$ & $\begin{array}{l}\text { Frequency of } \\
\text { publication }\end{array}$ \\
\hline Exchange Rates ${ }^{2}$ & NA & NA & NA & NA & NA \\
\hline International Reserve Assets and Reserve Liabilities of the Monetary Authorities ${ }^{3}$ & Feb 2009 & Apr 2009 & M & M, with 2- to 3-month lag & $\mathrm{A} / \mathrm{Q}$ \\
\hline Reserve/Base Money & Feb 2009 & Apr 2009 & M & M, with 2- to 3-month lag & $\mathrm{A} / \mathrm{Q}$ \\
\hline Broad Money & Feb 2009 & Apr 2009 & M & M, with 1- to 2-month lag & $\mathrm{A} / \mathrm{Q}$ \\
\hline Central Bank Balance Sheet & Feb 2009 & Apr 2009 & M & M, with 1- to 2-month lag & $\mathrm{A} / \mathrm{Q}$ \\
\hline Consolidated Balance Sheet of the Banking System & Feb 2009 & Apr 2009 & M & M, with 2- to 3-month lag & $\mathrm{A} / \mathrm{Q}$ \\
\hline Interest Rates ${ }^{4}$ & Feb 2009 & Apr 2009 & M & M, with 1- to 2-month lag & $\mathrm{A} / \mathrm{Q}$ \\
\hline Consumer Price Index & Mar 2009 & Apr 2009 & M & M, with 1- to 2-month lag & $\mathrm{A} / \mathrm{M}$ \\
\hline Revenue, Expenditure, Balance and Composition of Financing ${ }^{5}-$ Central Government & Mar 2009 & May 2009 & M & Q, with 1- to 2-month lag & A \\
\hline Stocks of Central Government and Central Government-Guaranteed Debt ${ }^{6}$ & Dec 2008 & Mar 2009 & $\mathrm{M} / \mathrm{A}$ & Q, with 1- to 2-month lag & A \\
\hline External Current Account Balance & Dec 2008 & Mar 2009 & A & A, with long lag & A \\
\hline Exports and Imports of Goods and Services & Dec 2008 & Mar 2009 & A & A, with long lag & A \\
\hline GDP/GNP & Dec 2008 & Mar 2009 & A & Staff Mission & A \\
\hline Gross External Debt ${ }^{7}$ & Dec 2008 & Mar 2009 & Q & Q, with 1-month lag & A/Semi-annual \\
\hline International Investment Position & NA & NA & NA & NA & NA \\
\hline
\end{tabular}

${ }^{1}$ Daily (D), Weekly (W), Monthly (M), Quarterly (Q), Annually (A); Irregular (I); Not Available (NA).

${ }^{2}$ Grenada is a member of the Eastern Caribbean Currency Union, in which the common currency of all member states (EC dollar) is pegged to the U.S. dollar.

${ }^{3}$ Includes reserve assets pledged or otherwise encumbered as well as net derivative positions.

${ }^{4}$ Both market-based and officially-determined, including discount rates, money market rates, rates on treasury bills, notes and bonds.

${ }^{5}$ Foreign, domestic bank, and domestic nonbank financing.

${ }^{6}$ Including currency and maturity composition.

${ }^{7}$ Public external debt only. 
June 4, 2009

\section{IMF Executive Board Completes Third Review Under PRGF Arrangement with Grenada, and Approves US\$6.8 Million Increase in Financial Assistance and US\$6 Million Disbursement}

The Executive Board of the International Monetary Fund (IMF) has completed the third review of Grenada's economic performance under the Poverty Reduction and Growth Facility (PRGF) arrangement and approved to increase financial assistance under the arrangement by an amount equivalent to SDR 4.39 million (about US\$6.8 million) to a total of SDR 16.38 million (about US\$25.4 million) to help Grenada cope with the adverse effects of the global slowdown and financial turmoil. The completion of the review allows for the immediate disbursement of an amount equivalent to SDR 3.875 million (about US\$6 million), bringing total disbursements to SDR 10.825 million (about US\$16.8 million).

The Executive Board also approved the request to modify a quantitative performance criterion by relaxing the target for the end-June 2009 primary balance excluding grants because of lower revenues as a result of the slowdown in growth. The Board also approved the completion of the financing assurances review.

The three-year PRGF with Grenada was approved on April 17, 2006 (see Press Release No. 06/75), augmented in July 2008 to SDR 11.99 million (about US\$18.6 million), and extended by one year to April 16, 2010.

Following the Executive Board discussion, Mr. Takatoshi Kato, Deputy Managing Director and Acting Chair, made the following statement:

"Grenada has resolutely implemented its PRGF-supported economic program in the face of economic difficulties arising from the global economic downturn. Since the second half of 2008, tourism earnings, foreign direct investment, and remittances have declined, which has led to a marked fall in economic growth and a projected shortfall in government revenue in 2009. The authorities have strengthened policies in response - in particular, they have 
tightened fiscal policy since the second half of 2008. The IMF is providing additional financing to support the government's adjustment efforts.

"To address the worsened budgetary outlook, the government is taking measures to contain public spending and increase spending efficiency. The authorities have announced the planned introduction of a value added tax in early 2010 to widen the tax base and increase revenue buoyancy. They are moving forward to introduce a market-based property tax and to improve tax administration.

"At the same time, some economic stimulus is being provided to sustain growth, mainly through an acceleration of priority capital spending and an increase in targeted social spending to mitigate the impact of economic slowdown on vulnerable groups. In that regard, it will be important to complete the Country Poverty Assessment without further delay.

"The authorities have made progress in ongoing institutional reform to improve economic management, including the recent establishment of a Debt Management Unit, a Private Sector Development Office, and a Waste Reduction Unit. They are committed to move forward with a more focused structural reform agenda, including the implementation of a customs reform strategy and measures to improve the business and investment environment.

"Though the banking sector has remained resilient, the authorities are carefully monitoring financial sector vulnerabilities. They have cooperated closely with regional governments to contain the fallout from the Trinidad and Tobago-based CL Financial Group, and plan to continue strengthening nonbank financial supervision and regulation.

"Grenada remains at high risk of debt distress. Any additional borrowing, even on concessional terms, would need to be carefully assessed so as not to undermine progress toward debt sustainability. The authorities are encouraged to build on recent progress in strengthening debt management capacity, and to continue efforts to regularize financial relations with external creditors," Mr. Kato stated. 